Center for

Mathematical Economics

Working Papers

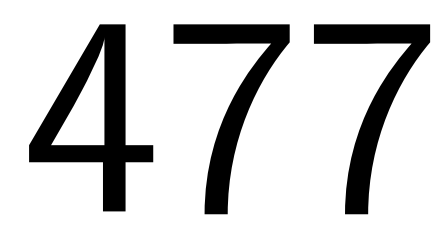

March 2013

\title{
A Stochastic Reversible Investment Problem on a Finite-Time Horizon: Free Boundary Analysis
}

Tiziano De Angelis and Giorgio Ferrari

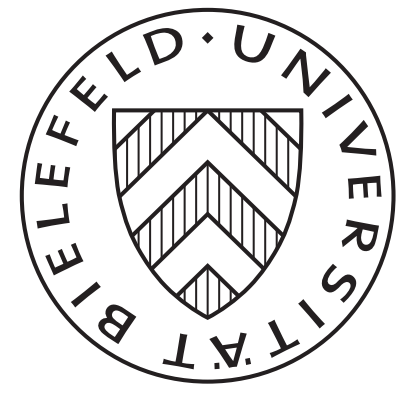




\title{
A Stochastic Reversible Investment Problem on a Finite-Time Horizon: Free Boundary Analysis*
}

\author{
Tiziano De Angelis ${ }^{\dagger} \quad$ Giorgio Ferrari ${ }^{\ddagger}$
}

March 25, 2013

\begin{abstract}
We study a continuous-time, finite horizon optimal stochastic reversible investment problem for a firm producing a single good. The production capacity is modeled as a onedimensional, time-homogeneous, linear diffusion controlled by a bounded variation process which represents the cumulative investment-disinvestment strategy. We associate to the investmentdisinvestment problem a zero-sum optimal stopping game and characterize its value function through a free boundary problem with two moving boundaries. These are continuous, bounded and monotone curves that solve a system of non-linear integral equations of Volterra type. The optimal investment-disinvestment strategy is then shown to be a diffusion reflected at the two boundaries.
\end{abstract}

Key words: reversible investment; singular stochastic control; zero-sum optimal stopping games; free boundary problems; Skorokhod reflection problem.

MSC2010 subsject classification: 93E20, 60G40, 35R35, 91A15, 91B70.

JEL classification: C02, C73, E22, D92.

\section{Introduction}

A firm represents the productive sector of a stochastic economy over a finite time horizon and it adjusts its production capacity $C$ by investing and disinvesting. The firm aims to maximizing its total net expected profit. In mathematical terms, following for instance [31], this amounts to solving

$$
\begin{gathered}
\sup _{\left(\nu_{+}, \nu_{-}\right)} \mathbb{E}\left\{\int_{0}^{T} e^{-\mu_{F} t} R\left(C^{y, \nu}(t)\right) d t-c_{+} \int_{0}^{T} e^{-\mu_{F} t} d \nu_{+}(t)+c_{-} \int_{0}^{T} e^{-\mu_{F} t} d \nu_{-}(t)\right. \\
\left.+e^{-\mu_{F} T} G\left(C^{y, \nu}(T)\right)\right\},
\end{gathered}
$$

where the optimization is taken over all the nondecreasing processes $\nu_{+}$and $\nu_{-}$representing the investment and disinvestment strategy, respectively. Here $\mu_{F}$ is the firm's manager discount factor, $c_{+}$is the istantaneous cost of investment, $c_{-}$is the benefit from disinvestment, $R$ the operating profit function and $G$ a terminal gain, often referred to as a scrap function. We assume that the

\footnotetext{
${ }^{*}$ The first author was partially supported by EPSRC grant EP/K00557X/1; Financial support by the German Research Foundation (DFG) via grant Ri-1128-4-1 is gratefully acknowledged by the second author.

${ }^{\dagger}$ School of Mathematics, University of Manchester, Oxford Rd. M13 9PL Manchester, UK; tiziano.deangelis@manchester.ac.uk

${ }^{\ddagger}$ Center for Mathematical Economics, Bielefeld University, Universitätsstraße 25, D-33615 Bielefeld, Germany; giorgio.ferrari@uni-bielefeld.de
} 
production capacity $C^{y, \nu}$ follows a time-homogeneous, linear controlled dynamics with $\nu:=\nu_{+}-\nu_{-}$ (cf. (2.1) below).

In this work we prove existence and uniqueness of the optimal solution pair $\left(\nu_{+}^{*}, \nu_{-}^{*}\right)$ to problem (1.1). Moreover, we provide a semi-explicit representation of this couple in terms of two continuous, bounded and monotone free boundaries which are characterized through a system of non-linear integral equations of Volterra type. To the best of our knowledge, integral equations for the free boundaries of zero-sum optimal stopping games on finite time-horizon have not received significant attention so far.

Theory of investment under uncertainty has received increasing attention in the last years in Economics as well as in Mathematics (see, for example, the extensive review in Dixit and Pindyck [22]). Several Authors studied the firm's optimal problem of capacity irreversible expansion via a number of different approaches. These include dynamic programming techniques (see [17], [36], [44], [49] and [56], among others), stochastic first-order conditions and the Bank-El Karoui's Representation Theorem [4] (see, e.g., [5], [18], [20], [26] and [59]), connections with optimal switching problems (cf. [32], among others). Models involving both expansion and reduction of a project's capacity level (i.e., reversible investment problems) have been recently considered by [1], [9], [31], [35], [45] and [47], among others. In [47], for example, an infinite time horizon problem of determining the optimal investment-disinvestment strategy that a firm should adopt in the presence of random price and/or demand fluctuations is considered. On the other hand, in [31] the Authors address a one-dimensional, infinite time horizon partially reversible investment model with entry decisions and a general running payoff function. They study the problem via a dynamic programming approach and characterize the optimal policy as a diffusion reflected along two (constant in time) boundaries. Here we consider the model of [31] without entry decision but with a finite-time horizon.

From the mathematical point of view our problem (1.1) falls within the class of bounded variation follower problems with finite horizon. These are singular stochastic control problems in which control processes are singular (as functions of time) with respect to the Lebesgue measure. The link existing between singular stochastic control, optimal stopping and free boundary problems has been thoroughly studied. Early papers by El Karoui and Karatzas [25], Karatzas [40] and Karatzas and Shreve [41] showed that the optimal control problem for a Brownian motion tracked by a nondecreasing process (i.e., the monotone follower control problem) is closely related to an auxiliary optimal stopping problem. In fact, the value function $V$ of the control problem is linked to the value function $v$ of the optimal stopping problem via $V_{y}=v$, where $V_{y}$ is the derivative of $V$ with respect to the space variable $y$. Later on, this link has been extended to more general controlled dynamics (see, e.g., [3], [12]). Recently, bounded variation control problems were brought into contact with optimal stopping games in a similar way (cf., for instance, Boetius [13], Chiarolla and Haussmann [15] and [16] and Karatzas and Wang [43]). In fact, in this setting one has $V_{y}=v$, with $v$ the saddle point of a Dynkin game, i.e. of a zero-sum optimal stopping game.

The analytical theory of stochastic differential games with stopping times has been developed by Bensoussan and Friedman [6], [7] and Friedman [28], among others. In a Markovian setting these Authors studied the saddle point of such games via the theory of partial differential equations (PDE), variational inequalities and free-boundary problems (see also the monographies by Bensoussan and Lions [8] and Friedman [30]). On the other hand, many papers tackled stochastic games of timing via probabilistic techniques: martingale approach was used for instance in [2], [11], [23] and [48]; Markovian structures were considered in [24] and [60], among others; a connection with stochastic backward differential equations may be found for example in [21], [33] and [34].

In this paper we use the link between bounded variation follower problems and zero-sum optimal stopping games to study problem (1.1). That is, we study the zero-sum optimal stopping game 
(Dynkin game) with value function

$$
\begin{aligned}
v(t, y):= & \inf _{\sigma \in[0, T-t]} \sup _{\tau \in[0, T-t]} \mathbb{E}\left\{\frac{c_{+}}{f_{C}} e^{-\mu_{F} \sigma} C^{0}(\sigma) \mathbb{1}_{\{\sigma \leq \tau\}} \mathbb{1}_{\{\sigma<T-t\}}+\frac{c_{-}}{f_{C}} e^{-\mu_{F} \tau} C^{0}(\tau) \mathbb{1}_{\{\tau<\sigma\}}\right. \\
& \left.+\int_{0}^{\tau \wedge \sigma} e^{-\mu_{F} s} C^{0}(s) R_{c}\left(y C^{0}(s)\right) d s+e^{-\mu_{F}(T-t)} C^{0}(T-t) G_{c}\left(y C^{0}(T-t)\right) \mathbb{1}_{\{\tau=\sigma=T-t\}}\right\}
\end{aligned}
$$

which is naturally associated to (1.1). Our analysis is carried out in several steps by means of arguments borrowed from probability and PDE theory. Setting $G(C)=\frac{c_{-}}{f_{C}} C$ in (1.1) we have $G_{C}(C)=\frac{c_{-}}{f_{C}}$ in $(1.2)$ and we meet usual conditions in the literature on variational methods applied to stochastic games (cf., e.g., [30], Chapter 16, Section 9).

We show that $v$ is a bounded, continuous function on $[0, T] \times(0, \infty)$ and that the state space $(t, y) \in[0, T] \times(0, \infty)$ splits into three regions defined via two continuous, bounded and monotone free boundaries $\hat{y}_{+}$and $\hat{y}_{-}$. The triple $\left(v, \hat{y}_{+}, \hat{y}_{-}\right)$solves a free boundary problem on $[0, T] \times(0, \infty)$ and $v$ fulfills the so-called smooth-fit property along the free boundaries (cf., e.g., [53]). We use local time-space calculus (cf. [52]) to show that $\left(v, \hat{y}_{+}, \hat{y}_{-}\right)$uniquely solves a system of non-linear integral equations of Volterra type under suitable boundary conditions (see Theorem 3.14 and Theorem 3.15 below).

The optimal control $\nu^{*}:=\nu_{+}^{*}-\nu_{-}^{*}$ for problem (1.1) turns out to be the minimal effort needed to keep the optimally controlled diffusion inside the closure of the region between the two free boundaries. Indeed, an applications of results in [14] allows us to prove that the optimally controlled capacity $C^{y, \nu^{*}}$ uniquely solves a Skorokhod reflection problem in the time-dependent interval $\left[\hat{y}_{+}(t), \hat{y}_{-}(t)\right], t<T$. Finally, we obtain a semi-explicit expression of the optimal control $\nu^{*}$.

The paper is organized as follows. In Section 2 we introduce the reversible investment problem and we prove existence and uniqueness of the optimal control. In Section 3 we study the associated zero-sum optimal stopping game by means of a probabilistic approach to free boundary problems. In particular in this Section we obtain the system of integral equations for $\left(v, \hat{y}_{+}, \hat{y}_{-}\right)$mentioned above. Finally, in Section 4 we find the optimal control strategy and Appendix A contains some technical proofs.

\section{The Reversible Investment Problem}

A firm represents the productive sector of a stochastic economy on a complete probability space $(\Omega, \mathcal{F}, \mathbb{P})$. We consider an exogeneous Brownian motion $W:=\{W(t), t \geq 0\}$ and denote by $\mathbb{F}:=\left\{\mathcal{F}_{t}, t \geq 0\right\}$ its natural filtration augmented by $\mathbb{P}$-null sets. Our setting is similar to the one in [31] but with finite time-horizon and no entry decision. The firm produces at rate $R(C)$ when its own capacity is $C$. We assume that the firm can either invest or disinvest in the market and we denote by $\nu_{+}(t)\left(\nu_{-}(t)\right)$ the cumulative investment (disinvestment) up to time $t$. Both $\nu_{+}$and $\nu-$ are left-continuous, a.s. finite, nondecreasing processes. Once the firm's manager adopts an investment-disinvestment strategy $\nu:=\nu_{+}-\nu_{-}$, then the production capacity evolves according to

$$
\left\{\begin{array}{l}
d C^{y, \nu}(t)=C^{y, \nu}(t)\left[-\mu_{C} d t+\sigma_{C} d W(t)\right]+f_{C} d \nu(t), \quad t \geq 0, \\
C^{y, \nu}(0)=y>0,
\end{array}\right.
$$

where $\mu_{C}, \sigma_{C}$ and $f_{C}$ are given positive constants. The parameter $f_{C}$ is a conversion factor: any unit of investment is converted into $f_{C}$ units of production capacity.

Notice that if

$$
C^{0}(t):=C^{1,0}(t), \quad \bar{\nu}(t):=\int_{0}^{t} \frac{f_{C}}{C^{0}(s)} d \nu(s)
$$


then we may write

$$
C^{y, \nu}(t)=C^{0}(t)[y+\bar{\nu}(t)]
$$

Moreover, $C^{0}$ represents the decay of a unit of initial capital without investment and we have

$$
C^{0}(t)=e^{-\mu_{C} t} \mathcal{M}_{0}(t)
$$

with $\mathcal{M}_{0}$ being the exponential martingale

$$
\mathcal{M}_{0}(t):=e^{-\frac{1}{2} \sigma_{C}^{2} t+\sigma_{C} W(t)}, \quad t \geq 0 .
$$

The production function of the firm is a nonnegative, measurable function $R: \mathbb{R}_{+} \mapsto \mathbb{R}_{+}$of the production capacity and it satisfies the following assumption.

Assumption 2.1. The mapping $C \mapsto R(C)$ is nondecreasing with $R(0)=0$ and strictly concave. It is twice continuously differentiable on $(0, \infty)$ and it has first derivative $R_{c}(C):=\frac{\partial}{\partial C} R(C)$ satisfying the Inada conditions

$$
\lim _{C \rightarrow 0} R_{c}(C)=\infty, \quad \lim _{C \rightarrow \infty} R_{c}(C)=0 .
$$

Denote by

$$
\mathcal{S}:=\left\{\nu: \Omega \times \mathbb{R}_{+} \mapsto \mathbb{R}_{+} \text {, of bounded variation, left-continuous, adapted s.t. } \nu(0)=0, \mathbb{P}-\text { a.s. }\right\}
$$

the nonempty, convex set of investment-disinvestment processes and from now on let $\nu_{+}-\nu_{-}$be the minimal decomposition of any admissible $\nu \in \mathcal{S}$ into the difference of two left-continuous, nondecreasing, adapted processes such that $\nu_{ \pm}(0)=0$ a.s.

We assume that the optimization runs over a finite-time horizon $[0, T]$. Starting at time zero and following an investment-disinvestment strategy $\nu \in \mathcal{S}$, the firm receives at terminal time $T$ a (discounted) payoff given by $e^{-\mu_{F} T} G\left(C^{y, \nu}(T)\right) . G$ is the so-called scrap value of the control problem. We assume that $G: \mathbb{R}_{+} \mapsto \mathbb{R}_{+}$is a strictly concave, nondecreasing, continuously differentiable function with first order derivative such that

$$
\frac{c_{-}}{f_{C}} \leq G_{c}(C) \leq \frac{c_{+}}{f_{C}}
$$

Here $c_{+}>c_{-}>0$ are the cost of investment and the benefit from disinvestment, respectively. Then, the firm's total expected profit, net of the costs, is given by

$$
\begin{gathered}
\mathcal{J}_{0, y}(\nu)=\mathbb{E}\left\{\int_{0}^{T} e^{-\mu_{F} t} R\left(C^{y, \nu}(t)\right) d t-c_{+} \int_{0}^{T} e^{-\mu_{F} t} d \nu_{+}(t)+c_{-} \int_{0}^{T} e^{-\mu_{F} t} d \nu_{-}(t)\right. \\
\left.+e^{-\mu_{F} T} G\left(C^{y, \nu}(T)\right)\right\},
\end{gathered}
$$

where $\mu_{F}>0$ is the firm's manager discount factor. The value $V$ of the optimal investmentdisinvestment problem is

$$
V(0, y):=\sup _{\nu \in \mathcal{S}} \mathcal{J}_{0, y}(\nu) .
$$

Notice that the strict concavity of $R$ and the affine nature of $C^{y, \nu}$ in $\nu$ imply that $\mathcal{J}_{0, y}(\nu)$ is strictly concave on $\mathcal{S}$. Hence, if a solution $\nu^{*}$ of $(2.8)$ exists, it is unique.

Proposition 2.2. Let Assumption 2.1 hold. Then, there exists $K:=K(T, y)>0$, depending on $T$ and $y$, such that $0 \leq V(0, y) \leq K$. 
Proof. Nonnegativity of $V(0, y)$ follows by taking $\nu_{+}(t)=\nu_{-}(t) \equiv 0$, for $t \geq 0$. To show that $V$ is bounded from above, recall that $C^{0}(t)=e^{-\mu_{F} t} \mathcal{M}_{0}(t)$ (cf. (2.4)) and that for any $\epsilon>0$ there exists $\kappa_{\epsilon}$ such that $R(C) \leq \kappa_{\epsilon}+\epsilon C$, by Inada conditions (cf. Assumption 2.1). Also there exists $\kappa_{G} \geq 0$ s.t. $G(C) \leq \kappa_{G}+\frac{c_{+}}{f_{C}} C$ by (2.6). Hence, setting $\bar{\mu}:=\mu_{F}+\mu_{C}$, for $\nu \in \mathcal{S}$ and $\bar{\nu}$ as in (2.2), we may write

$$
\begin{aligned}
& \mathcal{J}_{0, y}(\nu) \leq \mathbb{E}\left\{\int_{0}^{T} e^{-\mu_{F} t}\left[\kappa_{\epsilon}+\epsilon C^{y, \nu}(t)\right] d t-\frac{c_{+}}{f_{C}} \int_{0}^{T} e^{-\bar{\mu} t} \mathbb{E}\left\{\mathcal{M}_{0}(T) \mid \mathcal{F}_{t}\right\} d \bar{\nu}_{+}(t)\right. \\
&\left.+\frac{c_{-}}{f_{C}} \int_{0}^{T} e^{-\bar{\mu} t} \mathbb{E}\left\{\mathcal{M}_{0}(T) \mid \mathcal{F}_{t}\right\} d \bar{\nu}_{-}(t)+\kappa_{G}+\frac{c_{+}}{f_{C}} e^{-\bar{\mu} T} \mathcal{M}_{0}(T)\left[y+\bar{\nu}_{+}(T)-\bar{\nu}_{-}(T)\right]\right\} \\
& \leq \kappa_{\epsilon} T+ \frac{c_{+}}{f_{C}} y+\kappa_{G}+\epsilon y \mathbb{E}\left\{\int_{0}^{T} e^{-\bar{\mu} t} \mathcal{M}_{0}(t) d t\right\}+\epsilon \mathbb{E}\left\{\int_{0}^{T} e^{-\bar{\mu} t} \mathcal{M}_{0}(t) \bar{\nu}_{+}(t) d t\right\} \\
&-\epsilon \mathbb{E}\left\{\int_{0}^{T} e^{-\bar{\mu} t} \mathcal{M}_{0}(t) \bar{\nu}_{-}(t) d t\right\}-\frac{c_{+}}{f_{C}} \mathbb{E}\left\{\int_{0}^{T} e^{-\bar{\mu} t} \mathbb{E}\left\{\mathcal{M}_{0}(T) \mid \mathcal{F}_{t}\right\} d \bar{\nu}_{+}(t)\right\} \\
&+\frac{c_{-}}{f_{C}} \mathbb{E}\left\{\int_{0}^{T} e^{-\bar{\mu} t} \mathbb{E}\left\{\mathcal{M}_{0}(T) \mid \mathcal{F}_{t}\right\} d \bar{\nu}_{-}(t)\right\} \\
&+\frac{c_{+}}{f_{C}} \mathbb{E}\left\{e^{-\bar{\mu} T} \mathcal{M}_{0}(T)\left[\bar{\nu}_{+}(T)-\bar{\nu}_{-}(T)\right]\right\}
\end{aligned}
$$

Notice now that $\mathbb{E}\left\{\int_{[0, T)} e^{-\bar{\mu} t} \mathbb{E}\left\{\mathcal{M}_{0}(T) \mid \mathcal{F}_{t}\right\} d \bar{\nu}_{ \pm}(t)\right\}=\mathbb{E}\left\{\mathcal{M}_{0}(T) \int_{[0, T)} e^{-\bar{\mu} t} d \bar{\nu}_{ \pm}(t)\right\}$, by [38], Theorem 1.33 , and introduce the new probability measure $\tilde{\mathbb{P}}$ defined by

$$
\left.\frac{d \tilde{\mathbb{P}}}{d \mathbb{P}}\right|_{\mathcal{F}_{t}}:=\mathcal{M}_{0}(t)=e^{-\frac{1}{2} \sigma_{C}^{2} t+\sigma_{C} W(t)}, t \geq 0
$$

Then, integrating by parts the integrals with respect to $d \nu_{ \pm}$, we obtain from (2.9) that

$$
\begin{aligned}
& \mathcal{J}_{0, y}(\nu) \leq\left(\kappa_{\epsilon} T+\frac{c_{+}}{f_{C}} y+\kappa_{G}+\epsilon y T\right)+\epsilon \tilde{\mathbb{E}}\left\{\int_{0}^{T} e^{-\bar{\mu} t} \bar{\nu}_{+}(t) d t\right\}-\epsilon \tilde{\mathbb{E}}\left\{\int_{0}^{T} e^{-\bar{\mu} t} \bar{\nu}_{-}(t) d t\right\} \\
& -\frac{c_{+} \bar{\mu}}{f_{C}} \tilde{\mathbb{E}}\left\{\int_{0}^{T} e^{-\bar{\mu} t} \bar{\nu}_{+}(t) d t\right\}+\frac{c_{-} \bar{\mu}}{f_{C}} \tilde{\mathbb{E}}\left\{\int_{0}^{T} e^{-\bar{\mu} t} \bar{\nu}_{-}(t) d t\right\} \\
& +\frac{c_{-}}{f_{C}} \tilde{\mathbb{E}}\left\{e^{-\bar{\mu} T} \bar{\nu}_{-}(T)\right\}-\frac{c_{+}}{f_{C}} \tilde{\mathbb{E}}\left\{e^{-\bar{\mu} T} \bar{\nu}_{-}(T)\right\} \\
& \leq\left(\kappa_{\epsilon} T+\frac{c_{+}}{f_{C}} y+\kappa_{G}+\epsilon y T\right)+\left(\epsilon-\frac{c_{+} \bar{\mu}}{f_{C}}\right) \tilde{\mathbb{E}}\left\{\int_{0}^{T} e^{-\bar{\mu} t} \bar{\nu}_{+}(t) d t\right\} \\
& +\left(\frac{c_{-} \bar{\mu}}{f_{C}}-\epsilon\right) \tilde{\mathbb{E}}\left\{\int_{0}^{T} e^{-\bar{\mu} t} \bar{\nu}_{-}(t) d t\right\}+\left(\frac{c_{-}}{f_{C}}-\frac{c_{+}}{f_{C}}\right) \tilde{\mathbb{E}}\left\{e^{-\bar{\mu} T} \bar{\nu}_{-}(T)\right\} \\
& \leq K+\left(\epsilon-\frac{c_{+} \bar{\mu}}{f_{C}}\right) \tilde{\mathbb{E}}\left\{\int_{0}^{T} e^{-\bar{\mu} t} \bar{\nu}_{+}(t) d t\right\}+\left(\frac{c_{-} \bar{\mu}}{f_{C}}-\epsilon\right) \tilde{\mathbb{E}}\left\{\int_{0}^{T} e^{-\bar{\mu} t} \bar{\nu}_{-}(t) d t\right\},
\end{aligned}
$$

with $\tilde{\mathbb{E}}\{\cdot\}$ denoting the expectation under $\tilde{\mathbb{P}}$ and $K$ a positive constant independent of $\nu_{ \pm}$but depending on $y, T, \epsilon, c_{+}, c_{-}, f_{C}, \kappa_{G}$. Taking $\epsilon=\frac{c_{+} \bar{\mu}}{f_{C}}$, it follows

$$
\mathcal{J}_{0, y}(\nu) \leq K \quad \text { for all } \nu \in \mathcal{S},
$$

since $c_{+}>c_{-}$and $\bar{\nu}_{-}(t) \geq 0$ a.s. for every $t \geq 0$.

Corollary 2.3. There exist $K_{+}:=K_{+}(T, y)>0$ and $K_{-}:=K_{-}(T, y)>0$, depending on $T$ and $y$, such that $\tilde{\mathbb{E}}\left\{\bar{\nu}_{+}(T)\right\} \leq K_{+}$and $\tilde{\mathbb{E}}\left\{\bar{\nu}_{-}(T)\right\} \leq K_{-}$. 
Proof. Recall (2.10), then from (2.7), (2.8) and (2.12) we have

$$
K \geq V(0, y) \geq \mathcal{J}_{0, y}(\nu) \geq \tilde{\mathbb{E}}\left\{-\frac{c_{+}}{f_{C}} \int_{0}^{T} e^{-\bar{\mu} t} d \bar{\nu}_{+}(t)+\frac{c_{-}}{f_{C}} \int_{0}^{T} e^{-\bar{\mu} t} d \bar{\nu}_{-}(t)\right\}
$$

for any $\bar{\nu}_{+}$and $\bar{\nu}_{-}$left-continuous, adapted, nondecreasing such that $\nu_{ \pm}(0)=0$ a.s., as $R$ and $G$ are positive. Therefore, taking $\bar{\nu}_{-}:=f_{C}\left(\frac{c_{+}+1}{c_{-}}\right) \bar{\nu}_{+}$for any $\bar{\nu}_{+}$we find

$$
K \geq \tilde{\mathbb{E}}\left\{\int_{0}^{T} e^{-\bar{\mu} t} d \bar{\nu}_{+}(t)\right\}
$$

and taking $\bar{\nu}_{+} \equiv 0$ for arbitrary $\bar{\nu}_{-}$we find

$$
K \geq \tilde{\mathbb{E}}\left\{\frac{c_{-}}{f_{C}} \int_{0}^{T} e^{-\bar{\mu} t} d \bar{\nu}_{-}(t)\right\} .
$$

Thus, it finally follows from (2.14) and (2.15) that

$$
\tilde{\mathbb{E}}\left\{\bar{\nu}_{+}(T)\right\} \leq K e^{\bar{\mu} T}, \quad \tilde{\mathbb{E}}\left\{\bar{\nu}_{-}(T)\right\} \leq \frac{K f_{C}}{c_{-}} e^{\bar{\mu} T} .
$$

The next Theorem shows the existence of a unique optimal solution pair $\left(\nu_{+}^{*}, \nu_{-}^{*}\right)$ to problem $(2.8)$.

Theorem 2.4. Under Assumption 2.1 there exists a unique admissible investment-disinvestment strategy $\nu^{*}$ which is optimal for problem (2.8).

Proof. Note that the mapping $\nu \mapsto \bar{\nu}$ is one to one and onto. Let $\left(\nu^{n}\right)_{n \in \mathbb{N}} \subset \mathcal{S}$ be a maximizing sequence. The associated sequence $\left(\bar{\nu}^{n}\right)_{n \in \mathbb{N}} \subset \mathcal{S}$ is maximizing as well; that is, such that $\lim _{n \rightarrow \infty} \mathcal{J}_{0, y}\left(\bar{\nu}^{n}\right)=V(0, y)$. From Corollary 2.3 we have that the sequences $\left(\tilde{\mathbb{E}}\left\{\bar{\nu}_{ \pm}^{n}(T)\right\}\right)_{n \in \mathbb{N}}$ are uniformly bounded and hence by a version of Komlòs' Theorem for optional random measures on $[0, T]$ (cf. [39], Lemma 3.5) there exist two subsequences $\left(\bar{\nu}_{ \pm}^{n_{k}}\right)_{k \in \mathbb{N}}$ that converge weakly a.s. in the Cesàro sense to some optional measures $\bar{\nu}_{ \pm}^{*}$; i.e., if we define

$$
\theta_{ \pm}^{j}(t):=\frac{1}{j+1} \sum_{k=0}^{j} \bar{\nu}_{ \pm}^{n_{k}}(t),
$$

then

$$
\lim _{j \rightarrow \infty} \int_{0}^{T} f(t) d \theta_{ \pm}^{j}(t)=\int_{0}^{T} f(t) d \bar{\nu}_{ \pm}^{*}(t), \quad \tilde{\mathbb{P}}-\text { a.s. },
$$

for every continuous and bounded function $f(\cdot)$ (see, e.g., [10]). Moreover, a.s. weak convergence of $\theta_{ \pm}^{j}$ to $\bar{\nu}_{ \pm}^{*}$ (cf. $(2.18)$ ) is equivalent to having $\lim _{j \rightarrow \infty} \theta_{ \pm}^{j}(t)=\bar{\nu}_{ \pm}^{*}(t) \tilde{\mathbb{P}}$-a.s. for every point of continuity of $\bar{\nu}_{ \pm}^{*}(\cdot)$ and for $t=T$ (cf. [10]). Hence, $\lim _{j \rightarrow \infty} \theta_{ \pm}^{j}(t)=\bar{\nu}_{ \pm}^{*}(t)$ also $d \tilde{\mathbb{P}} \otimes d t$-a.e., as $\bar{\nu}_{ \pm}^{*}$ are left-continuous and nondecreasing.

Since $\left(\bar{\nu}^{n}\right)_{n \in \mathbb{N}}$ is a maximizing sequence, then $\left(\theta^{j}\right)_{j \in \mathbb{N}}, \theta^{j}:=\theta_{+}^{j}-\theta_{-}^{j}$, is maximizing as well by concavity of the profit functional. Now, if we could use (reverse) Fatou's Lemma, we would obtain

$$
V(0, y) \leq \limsup _{j \rightarrow \infty} J_{0, y}\left(\theta^{j}\right) \leq \mathcal{J}_{0, y}\left(\bar{\nu}^{*}\right)
$$

thus the optimality of $\nu_{ \pm}^{*}(t):=\int_{0}^{t} \frac{C^{0}(s)}{f_{C}} d \bar{\nu}_{ \pm}^{*}(s)$. Uniqueness follows as usual from strict concavity of $\mathcal{J}_{0, y}$ and convexity of $\mathcal{S}$. 
It remains to show that (reverse) Fatou's Lemma can be applied. Under the measure $\tilde{\mathbb{P}}$ we may write the net profit functional $\mathcal{J}_{0, y}$ in $(2.7)$ for any $\bar{\nu} \in \mathcal{S}$ as

$$
\begin{aligned}
\mathcal{J}_{0, y}(\bar{\nu})=\tilde{\mathbb{E}}\left\{\int_{0}^{T} e^{-\mu_{F} t} \frac{1}{\mathcal{M}_{0}(t)} R\left(C^{y, \bar{\nu}}(t)\right) d t-\frac{c_{+}}{f_{C}} \int_{0}^{T} e^{-\bar{\mu} t} d \bar{\nu}_{+}(t)+\frac{c_{-}}{f_{C}} \int_{0}^{T} e^{-\bar{\mu} t} d \bar{\nu}_{-}(t)\right. \\
\left.\quad+e^{-\mu_{F} T} \frac{1}{\mathcal{M}_{0}(T)} G\left(C^{y, \bar{\nu}}(T)\right)\right\} \\
=\tilde{\mathbb{E}}\left\{\int_{0}^{T}\left[e^{-\mu_{F} t} \frac{1}{\mathcal{M}_{0}(t)} R\left(C^{y, \bar{\nu}}(t)\right)-e^{-\bar{\mu} t}\left(\frac{c_{+} \bar{\mu}_{f_{C}}}{f_{+}}(t)-\frac{c_{-} \bar{\mu}}{f_{C}} \bar{\nu}_{-}(t)\right)\right] d t\right. \\
\left.-e^{-\bar{\mu} T}\left(\frac{c_{+}}{f_{C}} \bar{\nu}_{+}(T)-\frac{c_{-}}{f_{C}} \bar{\nu}_{-}(T)\right)+e^{-\mu_{F} T} \frac{1}{\mathcal{M}_{0}(T)} G\left(C^{y, \bar{\nu}}(T)\right)\right\} \\
=: \tilde{\mathbb{E}}\left\{\int_{0}^{T} \Phi^{y, \bar{\nu}}(t) d t+\hat{G}^{y, \bar{\nu}}(T)\right\},
\end{aligned}
$$

where we have performed an integration by parts to obtain the second equality, $\bar{\mu}=\mu_{C}+\mu_{F}$ and

$$
\begin{aligned}
\Phi^{y, \bar{\nu}}(t) & :=e^{-\mu_{F} t} \frac{1}{\mathcal{M}_{0}(t)} R\left(C^{y, \bar{\nu}}(t)\right)-e^{-\bar{\mu} t}\left(\frac{c_{+} \bar{\mu}}{f_{C}} \bar{\nu}_{+}(t)-\frac{c_{-} \bar{\mu}}{f_{C}} \bar{\nu}_{-}(t)\right) \\
\hat{G}^{y, \bar{\nu}}(T) & :=e^{-\mu_{F} T} \frac{1}{\mathcal{M}_{0}(T)} G\left(C^{y, \bar{\nu}}(T)\right)-e^{-\bar{\mu} T}\left(\frac{c_{+}}{f_{C}} \bar{\nu}_{+}(T)-\frac{c_{-}}{f_{C}} \bar{\nu}_{-}(T)\right) .
\end{aligned}
$$

Recall (2.3) and $c_{+}>c_{-}$. Since for every $\epsilon>0$ there exists $\kappa_{\epsilon}>0$ such that $R(C) \leq \kappa_{\epsilon}+\epsilon C$ (cf. Assumption 2.1), then we obtain

$$
\Phi^{y, \bar{\nu}}(t) \leq \frac{\kappa_{\epsilon} e^{-\mu_{F} t}}{\mathcal{M}_{0}(t)}+\epsilon y e^{-\bar{\mu} t}+e^{-\bar{\mu} t} \bar{\nu}_{+}(t)\left(\epsilon-\frac{c_{+} \bar{\mu}}{f_{C}}\right)+e^{-\bar{\mu} t \bar{\nu}_{-}}(t)\left(\frac{c_{-} \bar{\mu}}{f_{C}}-\epsilon\right), \quad \bar{\nu} \in \mathcal{S} .
$$

We now take $\epsilon=\frac{\bar{\mu} c_{-}}{f_{C}}$ and we find

$$
\Phi^{y, \bar{\nu}}(t) \leq \hat{K}\left(1+\frac{1}{\mathcal{M}_{0}(t)}\right),
$$

for some $\hat{K}>0$, and the right-hand side of (2.20) is $d \tilde{\mathbb{P}} \otimes d t$-integrable and independent of $\bar{\nu}$. Again, $G(C) \leq \frac{c_{+}}{f_{C}} C+\kappa_{G}$, for some $\kappa_{G} \geq 0$ (cf. (2.6)), and hence

$$
\hat{G}^{y, \bar{\nu}}(T) \leq \frac{\kappa_{G} e^{-\mu_{F} T}}{\mathcal{M}_{0}(T)}+\frac{c_{+} y}{f_{C}} e^{-\bar{\mu} T} .
$$

Note that the right-hand side of (2.21) is independent of $\bar{\nu}$ and $\tilde{\mathbb{P}}$-integrable. Therefore we can apply Fatou's Lemma to justify (2.19).

\section{The Zero-Sum Optimal Stopping Game}

In order to characterize the optimal control policy we shall associate to problem (2.8) a suitable zero-sum optimal stopping game, in the spirit of [23] and [43], among others. Then, we will show that the value function solves a free boundary problem with two free boundaries which are continuous, bounded and monotone solutions of a system of non-linear integral equations.

As usual in the literature of dynamic programming, we let the optimization in (2.8) start at arbitrary time $t \in[0, T]$. Since the solution of (2.1) and the net profit functional are timehomogeneous, then we may simply set a time horizon $[0, T-t]$ in $(2.7)$ and write

$$
\begin{aligned}
\mathcal{J}_{t, y}(\nu)=\mathbb{E}\left\{\int_{0}^{T-t}\right. & e^{-\mu_{F} s} R\left(C^{y, \nu}(s)\right) d s-c_{+} \int_{0}^{T-t} e^{-\mu_{F} s} d \nu_{+}(s)+c_{-} \int_{0}^{T-t} e^{-\mu_{F} s} d \nu_{-}(s) \\
& \left.+e^{-\mu_{F}(T-t)} G\left(C^{y, \nu}(T-t)\right)\right\}
\end{aligned}
$$


It follows that the firm's investment-disinvestment problem now reads

$$
V(t, y):=\sup _{\nu \in \mathcal{S}} \mathcal{J}_{t, y}(\nu)
$$

From (2.2) and (2.3), we may write the value function $V(t, y)$ of the optimal control problem (3.2) in terms of a maximization over the controls $\bar{\nu} \in \mathcal{S}$; that is,

$$
\begin{aligned}
V(t, y)=\sup _{\bar{\nu} \in \mathcal{S}} \mathbb{E}\left\{\int_{0}^{T-t}\right. & e^{-\mu_{F} s} R\left(C^{0}(s)[y+\bar{\nu}(s)]\right) d s-\frac{c_{+}}{f_{C}} \int_{0}^{T-t} e^{-\mu_{F} s} C^{0}(s) d \bar{\nu}_{+}(s) \\
& \left.+\frac{c_{-}}{f_{C}} \int_{0}^{T-t} e^{-\mu_{F} t} C^{0}(s) d \bar{\nu}_{-}(s)+e^{-\mu_{F}(T-t)} G\left(C^{0}(T-t)[y+\bar{\nu}(T-t)]\right)\right\} .
\end{aligned}
$$

In order to employ results by [43], take $\omega \in \Omega, s \in[0, T-t], y \in(0, \infty)$ and set

$$
\left\{\begin{array}{l}
\xi_{ \pm}(\omega, s):=\bar{\nu}_{ \pm}(\omega, s), \\
X(\omega, s):=y+\bar{\nu}(\omega, s)=y+\xi^{+}(\omega, s)-\xi^{-}(\omega, s), \\
H(\omega, s, y):=-e^{-\mu_{F} s} R\left(y C^{0}(\omega, s)\right), \\
\gamma(\omega, s):=\frac{c_{+}}{f_{C}} e^{-\mu_{F} s} C^{0}(\omega, s) \mathbb{1}_{\{s<T-t\}}, \quad \nu(\omega, s):=-\frac{c_{-}}{f_{C}} e^{-\mu_{F} s} C^{0}(\omega, s) \mathbb{1}_{\{s<T-t\}}, \\
G(\omega, y):=-e^{-\mu_{F}(T-t)} G\left(y C^{0}(\omega, T-t)\right) .
\end{array}\right.
$$

Notice that $H_{y}(\omega, s, y)$ is $d \mathbb{P} \otimes d t$-integrable for any $y>0$, thanks to concavity of $R$, whereas $G_{y}(\omega, y)$ is $d \mathbb{P}$-integrable by (2.6). Moreover $\mathbb{E}\left\{\sup _{0 \leq s \leq T-t}|\gamma(s)|+\sup _{0 \leq s \leq T-t}|\nu(s)|\right\}<\infty$. Then, thanks to [43], Theorem 2.3, we fit into [43], Theorem 3.2, (with time horizon $T-t$ ) and the following result holds.

Proposition 3.1. Under Assumption 2.1, the value function $V(t, y)$ of the control problem (3.2) satisfies

$$
\frac{\partial}{\partial y} V(t, y)=v(t, y), \quad(t, y) \in[0, T] \times(0, \infty)
$$

where

$$
\begin{aligned}
v(t, y):= & \inf _{\sigma \in[0, T-t]} \sup _{\tau \in[0, T-t]} \mathbb{E}\left\{\frac{c_{+}}{f_{C}} e^{-\mu_{F} \sigma} C^{0}(\sigma) \mathbb{1}_{\{\sigma \leq \tau\}} \mathbb{1}_{\{\sigma<T-t\}}+\frac{c_{-}}{f_{C}} e^{-\mu_{F} \tau} C^{0}(\tau) \mathbb{1}_{\{\tau<\sigma\}}\right. \\
& \left.\quad+\int_{0}^{\tau \wedge \sigma} e^{-\mu_{F} s} C^{0}(s) R_{c}\left(y C^{0}(s)\right) d s+e^{-\mu_{F}(T-t)} C^{0}(T-t) G_{C}\left(y C^{0}(T-t)\right) \mathbb{1}_{\{\tau=\sigma=T-t\}}\right\} \\
= & \sup _{\tau \in[0, T-t]} \inf _{\sigma \in[0, T-t]} \mathbb{E}\left\{\frac{c_{+}}{f_{C}} e^{-\mu_{F} \sigma} C^{0}(\sigma) \mathbb{1}_{\{\sigma \leq \tau\}} \mathbb{1}_{\{\sigma<T-t\}}+\frac{c_{-}}{f_{C}} e^{-\mu_{F} \tau} C^{0}(\tau) \mathbb{1}_{\{\tau<\sigma\}}\right. \\
& \left.+\int_{0}^{\tau \wedge \sigma} e^{-\mu_{F} s} C^{0}(s) R_{c}\left(y C^{0}(s)\right) d s+e^{-\mu_{F}(T-t)} C^{0}(T-t) G_{c}\left(y C^{0}(T-t)\right) \mathbb{1}_{\{\tau=\sigma=T-t\}}\right\} .
\end{aligned}
$$

Here $v(t, y)$ is the value function of a zero-sum optimal stopping game (Dynkin game). Consider two players, $\mathcal{P}_{1}$ and $\mathcal{P}_{2}$, starting playing at time $t \in[0, T]$. Player $\mathcal{P}_{1}$ can choose the stopping time $\sigma$, whereas player $\mathcal{P}_{2}$ the stopping time $\tau$. The game ends as soon as one of the two players decides to stop, i.e. at the stopping time $\sigma \wedge \tau$. As long as the game is in progress, $\mathcal{P}_{1}$ keeps paying $\mathcal{P}_{2}$ at the (random) rate $e^{-\mu_{F} t} C^{0}(t) R_{c}\left(y C^{0}(t)\right)$ per unit of time. When the game ends before $T-t, \mathcal{P}_{1}$ pays $\frac{c_{+}}{f_{C}} e^{-\mu_{F} \sigma} C^{0}(\sigma)$ if she/he decides to stop earlier than $\mathcal{P}_{2}$; otherwise $\mathcal{P}_{1}$ pays $\frac{c_{-}}{f_{C}} e^{-\mu_{F} \tau} C^{0}(\tau)$. If 
no one decides to stop the game (i.e. the game ends at $T-t$ ), $\mathcal{P}_{1}$ pays $\mathcal{P}_{2}$ the (random) amount $e^{-\mu_{F}(T-t)} C^{0}(T-t) G_{c}\left(y C^{0}(T-t)\right)$. It follows that the (random) total payment from $\mathcal{P}_{1}$ to $\mathcal{P}_{2}$ is

$$
\begin{aligned}
& \frac{c_{+}}{f_{C}} e^{-\mu_{F} \sigma} C^{0}(\sigma) \mathbb{1}_{\{\sigma \leq \tau\}} \mathbb{1}_{\{\sigma<T-t\}}+\frac{c_{-}}{f_{C}} e^{-\mu_{F} \tau} C^{0}(\tau) \mathbb{1}_{\{\tau<\sigma\}} \\
& \quad+\int_{0}^{\tau \wedge \sigma} e^{-\mu_{F} s} C^{0}(s) R_{c}\left(y C^{0}(s)\right) d s+e^{-\mu_{F}(T-t)} C^{0}(T-t) G_{c}\left(y C^{0}(T-t)\right) \mathbb{1}_{\{\tau=\sigma=T-t\}}
\end{aligned}
$$

Hence, as it is natural, $\mathcal{P}_{1}$ tries to minimize the expected value of (3.6), whereas $\mathcal{P}_{2}$ tries to maximize it.

Remark 3.2. Notice that in [43], Theorem 3.2, the instantaneous cost functions $\gamma$ and $\nu$ are both positive. This is not true in our setting, however reading carefully the proof of [43], Theorem 3.2, one can see that such condition is not necessary.

Recall now $\tilde{\mathbb{P}}$ defined in $(2.10)$ and set $\tilde{W}(t):=W(t)-\sigma_{C} t, t \geq 0$. This process is a $\tilde{\mathbb{P}}$-Brownian motion and

$$
C^{0}(t)=e^{\hat{\mu}_{C} t+\sigma_{C} \tilde{W}(t)},
$$

with $\hat{\mu}_{C}:=-\mu_{C}+\frac{1}{2} \sigma_{C}^{2}$, under the new measure. Then Girsanov Theorem allows us to rewrite $v(t, y)$ of $(3.5)$ under $\tilde{\mathbb{P}}$ as

$$
v(t, y):=\inf _{\sigma \in[0, T-t]} \sup _{\tau \in[0, T-t]} \Psi(t, y ; \sigma, \tau)=\sup _{\tau \in[0, T-t]} \inf _{\sigma \in[0, T-t]} \Psi(t, y ; \sigma, \tau),
$$

with

$$
\begin{aligned}
\Psi(t, y ; \sigma, \tau):=\tilde{\mathbb{E}}\{ & \frac{c_{+}}{f_{C}} e^{-\bar{\mu} \sigma} \mathbb{1}_{\{\sigma \leq \tau\}} \mathbb{1}_{\{\sigma<T-t\}}+\frac{c_{-}}{f_{C}} e^{-\bar{\mu} \tau} \mathbb{1}_{\{\tau<\sigma\}} \\
& \left.+e^{-\bar{\mu}(T-t)} G_{c}\left(y C^{0}(T-t)\right) \mathbb{1}_{\{\tau=\sigma=T-t\}}+\int_{0}^{\tau \wedge \sigma} e^{-\bar{\mu} s} R_{c}\left(y C^{0}(s)\right) d s\right\}
\end{aligned}
$$

and, again, $\bar{\mu}:=\mu_{C}+\mu_{F}$. Notice that

$$
\frac{c_{-}}{f_{C}} \leq v(t, y) \leq \frac{c_{+}}{f_{C}}
$$

for all $(t, y) \in[0, T] \times(0, \infty)$.

From now on, our aim will be to characterize the optimal control $\nu^{*}$ for problem (3.2) in terms of the optimal strategy of the zero-sum game (3.8). We expect the latter to be given by the first exit times $\left(\sigma^{*}, \tau^{*}\right)$ of the process $\left\{y C^{0}(s), s \geq 0\right\}$ from the region bounded between two moving boundaries denoted by $\hat{y}_{+}$and $\hat{y}_{-}$, respectively. A characterization of the free-boundaries is hard to find in general. However, that can be accomplished when the marginal scrap value $G_{c}$ coincide with either $\frac{c_{+}}{f_{C}}$ or $\frac{c_{-}}{f_{C}}$. That is a common assumption when addressing zero-sum optimal stopping games with variational methods (cf., e.g., [30], Chapter 16, Section 9). We observe that if $G_{c}(C)=\frac{c_{+}}{f_{C}}$, the player who aims to maximize $\Psi$ will choose a 'no-action strategy' for $t>\left[T-\frac{1}{\bar{\mu}} \ln \left(\frac{c_{+}}{c_{-}}\right)\right]^{+}$regardless of the initial state $y$. In fact, an immediate stopping would get her/him a reward equal to $\frac{c_{-}}{f_{C}}$, whereas doing nothing would guarantee a payoff larger than $\frac{c_{+}}{f_{C}} e^{-\bar{\mu}(T-t)}$. Somehow this introduces an advantage for the 'sup-player' as her/his strategy is known on a whole time interval before the end of the game. To avoid such a situation we make the following

Assumption 3.3. $G(C)=\frac{c_{-}}{f_{C}} C$.

Theorem 3.4. Under Assumptions 2.1 and 3.3 the value function $v(t, y)$ defined in (3.8) is continuous on $[0, T] \times(0, \infty)$. 
The full proof of this Theorem is quite technical and it is contained in Appendix A, Section A.1. It follows by adapting to the present setting arguments developed in [61]. Continuity of $v(t, y)$ on $[0, T] \times(0, \infty)$ is indeed obtained by introducing suitable penalized problems, by showing that their solutions $u^{\epsilon}, \epsilon>0$, are continuous and that they converge uniformly to $v$ as $\epsilon \downarrow 0$ on compact subsets of $[0, T] \times(0, \infty)$.

Theorem 3.5. Under Assumptions 2.1 and 3.3 the stopping times

$$
\left\{\begin{array}{l}
\sigma^{*}(t, y):=\inf \left\{s \in[0, T-t): v\left(t+s, y C^{0}(s)\right) \geq \frac{c_{+}}{f_{C}}\right\} \wedge(T-t), \\
\tau^{*}(t, y):=\inf \left\{s \in[0, T-t): v\left(t+s, y C^{0}(s)\right) \leq \frac{c_{-}}{f_{C}}\right\} \wedge(T-t),
\end{array}\right.
$$

are a saddle point for the zero-sum game (3.8).

Theorem 3.5 is proved in Appendix A, Section A.2. As a natural byproduct of its proof we obtain the following

Proposition 3.6. Take $(t, y) \in[0, T] \times(0, \infty)$ arbitrary but fixed and let $\rho \in[0, T-t]$ be any stopping time. Then under Assumptions 2.1 and 3.3 the value function $v$ satisfies

$$
\begin{array}{r}
\text { i) } \quad v(t, y) \leq \tilde{\mathbb{E}}\left\{e^{-\bar{\mu}\left(\rho \wedge \tau^{*}\right)} v\left(t+\rho \wedge \tau^{*}, y C^{0}\left(\rho \wedge \tau^{*}\right)\right)+\int_{0}^{\rho \wedge \tau^{*}} e^{-\bar{\mu} s} R_{c}\left(y C^{0}(s)\right) d s\right\} \\
\text { ii) } \quad v(t, y) \geq \tilde{\mathbb{E}}\left\{e^{-\bar{\mu}\left(\sigma^{*} \wedge \rho\right)} v\left(t+\sigma^{*} \wedge \rho, y C^{0}\left(\sigma^{*} \wedge \rho\right)\right)+\int_{0}^{\sigma^{*} \wedge \rho} e^{-\bar{\mu} s} R_{c}\left(y C^{0}(s)\right) d s\right\} \\
\text { iii }) \quad v(t, y)=\tilde{\mathbb{E}}\left\{e^{-\bar{\mu}\left(\rho \wedge \sigma^{*} \wedge \tau^{*}\right)} v\left(t+\rho \wedge \sigma^{*} \wedge \tau^{*}, y C^{0}\left(\rho \wedge \sigma^{*} \wedge \tau^{*}\right)\right)\right. \\
\left.+\int_{0}^{\rho \wedge \sigma^{*} \wedge \tau^{*}} e^{-\bar{\mu} s} R_{c}\left(y C^{0}(s)\right) d s\right\}
\end{array}
$$

Proof. Inequalities $i$ ) and $i i$ ) are direct consequences of (A-51) and (A-53), respectively. Equality iii) follows by exactly the same arguments as in (A-45)-(A-47).

The above characterization of the value function was also found via purely probabilistic methods in [55] and, in that paper, properties $i$ ), ii) and iii) were referred to as semi-harmonic characterization of $v$.

Proposition 3.7. Under Assumptions 2.1 and 3.3 the value function $v(t, y)$ is

1. decreasing in $y$ for each $t \in[0, T]$;

2. decreasing in $t$ for each $y \in(0, \infty)$.

Proof. 1. Fix $t \in[0, T]$ and $y_{1}>y_{2}>0$. Let $\left(\sigma_{1}^{*}, \tau_{1}^{*}\right)$ be optimal for $\left(t, y_{1}\right)$ and $\left(\sigma_{2}^{*}, \tau_{2}^{*}\right)$ be optimal for $\left(t, y_{2}\right)$. By definition of $v(t, y)$ (cf. (3.8)) we have

$$
\begin{aligned}
& v\left(t, y_{1}\right)-v\left(t, y_{2}\right) \leq \tilde{\mathbb{E}}\left\{\frac{c_{+}}{f_{C}} e^{-\bar{\mu} \sigma} \mathbb{1}_{\left\{\sigma \leq \tau_{1}^{*}\right\}} \mathbb{1}_{\{\sigma<T-t\}}+\frac{c_{-}}{f_{C}} e^{-\bar{\mu} \tau_{1}^{*}} \mathbb{1}_{\left\{\tau_{1}^{*}<\sigma\right\}}\right. \\
& \left.+\int_{0}^{\tau_{1}^{*} \wedge \sigma} e^{-\bar{\mu} s} R_{c}\left(y_{1} C^{0}(s)\right) d s+e^{-\bar{\mu}(T-t)} \frac{c_{-}}{f_{C}} \mathbb{1}_{\left\{\tau_{1}^{*}=\sigma=T-t\right\}}\right\} \\
& -\tilde{\mathbb{E}}\left\{\frac{c_{+}}{f_{C}} e^{-\bar{\mu} \sigma_{2}^{*}} \mathbb{1}_{\left\{\sigma_{2}^{*} \leq \tau\right\}} \mathbb{1}_{\left\{\sigma_{2}^{*}<T-t\right\}}+\frac{c_{-}}{f_{C}} e^{-\bar{\mu} \tau} \mathbb{1}_{\left\{\tau<\sigma_{2}^{*}\right\}}+\int_{0}^{\sigma_{2}^{*} \wedge \tau} e^{-\bar{\mu} s} R_{c}\left(y_{2} C^{0}(s)\right) d s\right. \\
& \left.+e^{-\bar{\mu}(T-t)} \frac{c_{-}}{f_{C}} \mathbb{1}_{\left\{\sigma_{2}^{*}=\tau=T-t\right\}}\right\},
\end{aligned}
$$


for any $\sigma$ and $\tau$ in $[0, T-t]$. If we now set $\sigma:=\sigma_{2}^{*}$ and $\tau:=\tau_{1}^{*}$, then we have

$$
v\left(t, y_{1}\right)-v\left(t, y_{2}\right) \leq \tilde{\mathbb{E}}\left\{\int_{0}^{\tau_{1}^{*} \wedge \sigma_{2}^{*}} e^{-\bar{\mu} s}\left[R_{c}\left(y_{1} C^{0}(s)\right)-R_{c}\left(y_{2} C^{0}(s)\right)\right] d s\right\} \leq 0
$$

as $R_{c}(\cdot)$ is decreasing.

2. Given $(t, y) \in[0, T] \times(0, \infty)$, for fixed $\theta \in[0, T-t]$ we define the ' $\theta$-shifted' value function as $v^{\theta}(t, y):=v(t+\theta, y)$. Introduce the stopping time

$$
\tau_{\theta}^{*}:=\inf \left\{s \in[0, T-t-\theta): v^{\theta}\left(t+s, y C^{0}(s)\right) \leq \frac{c_{-}}{f_{C}}\right\} \wedge(T-t-\theta),
$$

and note that it is optimal for the sup-problem in $v^{\theta}$. Recalling (3.11) and setting $\rho_{\theta}:=\sigma^{*} \wedge \tau_{\theta}^{*}$, then we obtain

$$
\tilde{\mathbb{E}}\left\{e^{-\bar{\mu} \rho_{\theta}}\left[v^{\theta}\left(t+\rho_{\theta}, y C^{0}\left(\rho_{\theta}\right)\right)-v\left(t+\rho_{\theta}, y C^{0}\left(\rho_{\theta}\right)\right)\right]\right\} \geq v^{\theta}(t, y)-v(t, y),
$$

by (3.12) and (3.13). In order to show that the left-hand side of (3.16) is negative, notice that

- on $\left\{\rho_{\theta}=T-t-\theta\right\}: v^{\theta}\left(T-\theta, y C^{0}(T-t-\theta)\right)=v\left(T, y C^{0}(T-t-\theta)\right)=\frac{c_{-}}{f_{C}}$ and, on the other hand, $v\left(T-\theta, y C^{0}(T-t-\theta)\right) \geq \frac{c_{-}}{f_{C}}$.

- on $\left\{\rho_{\theta}=\tau_{\theta}^{*}\right\} \bigcap\left\{\rho_{\theta}<T-t-\theta\right\}: v^{\theta}\left(t+\tau_{\theta}^{*}, y C^{0}\left(\tau_{\theta}^{*}\right)\right)=\frac{c_{-}}{f_{C}}$ and $v\left(t+\tau_{\theta}^{*}, y C^{0}\left(\tau_{\theta}^{*}\right) \geq \frac{c_{-}}{f_{C}}\right.$.

- on $\left\{\rho_{\theta}=\sigma^{*}\right\} \bigcap\left\{\rho_{\theta}<T-t-\theta\right\}: v^{\theta}\left(t+\sigma^{*}, y C^{0}\left(\sigma^{*}\right)\right) \leq \frac{c_{+}}{f_{C}}$ and $v\left(t+\sigma^{*}, y C^{0}\left(\sigma^{*}\right)\right)=\frac{c_{+}}{f_{C}}$.

It thus follows that $v(t+\theta, y) \leq v(t, y)$ for any $\theta \in[0, T-t]$ by (3.16).

We now define the continuation region

$$
\mathcal{C}:=\left\{(t, y) \in[0, T] \times(0, \infty): \frac{c_{-}}{f_{C}}<v(t, y)<\frac{c_{+}}{f_{C}}\right\},
$$

and the two stopping regions

$$
\begin{aligned}
& \mathcal{S}_{+}:=\left\{(t, y) \in[0, T] \times(0, \infty): v(t, y)=\frac{c_{+}}{f_{C}}\right\} \\
& \mathcal{S}_{-}:=\left\{(t, y) \in[0, T] \times(0, \infty): v(t, y)=\frac{c_{-}}{f_{C}}\right\} .
\end{aligned}
$$

Notice that $\mathcal{C}$ is an open subset of $[0, T] \times(0, \infty)$ and $\mathcal{S}_{+}, \mathcal{S}_{-}$are closed ones, due to continuity of $v$ (cf. Theorem 3.4) Moreover, for $t \in[0, T]$ fixed, denote by $\mathcal{C}_{t}:=\left\{y \in(0, \infty): \frac{c_{-}}{f_{C}}<v(t, y)<\frac{c_{+}}{f_{C}}\right\}$ the $t$-section of the continuation region. Analogously, we introduce the $t$-sections $\mathcal{S}_{+, t}, \mathcal{S}_{-, t}$ of the two stopping regions.

Proposition 3.8. Let Assumptions 2.1 and 3.3 hold. Then, for any $t \in[0, T]$, there exist $\hat{y}_{+}(t)<$ $\hat{y}_{-}(t)$ such that $\mathcal{C}_{t}=\left(\hat{y}_{+}(t), \hat{y}_{-}(t)\right) \subset[0, \infty], \mathcal{S}_{+, t}=\left[0, \hat{y}_{+}(t)\right]$ and $\mathcal{S}_{-, t}=\left[\hat{y}_{-}(t), \infty\right]$.

Proof. The result follows by 1 . of Proposition 3.7 and recalling that $\mathcal{C}$ is open. 
Since $y \mapsto v(t, y)$ is decreasing (cf. Proposition 3.7), then $\mathcal{S}_{+}$lies below $\mathcal{C}$, and $\mathcal{C}$ lies below $\mathcal{S}_{-}$. From Proposition 3.8 it is natural to define the two free boundaries as

$$
\hat{y}_{+}(t):=\sup \left\{y>0: v(t, y)=\frac{c_{+}}{f_{C}}\right\}
$$

and

$$
\hat{y}_{-}(t):=\inf \left\{y>0: v(t, y)=\frac{c_{-}}{f_{C}}\right\} .
$$

Remark 3.9. It is easy to see that the optimal stopping times $\tau^{*}$ and $\sigma^{*}$ of (3.11) may be written in terms of the free boundaries $\hat{y}_{+}$and $\hat{y}_{-}$of (3.17) and (3.18), respectively, as

$$
\left\{\begin{array}{l}
\tau^{*}(t, y):=\inf \left\{s \in[0, T-t):\left(\hat{y}_{-}(t+s)-y C^{0}(s)\right)^{+}=0\right\} \wedge(T-t), \\
\sigma^{*}(t, y):=\inf \left\{s \in[0, T-t):\left(y C^{0}(s)-\hat{y}_{+}(t+s)\right)^{+}=0\right\} \wedge(T-t) .
\end{array}\right.
$$

Recalling now Theorem 3.4, Theorem 3.5, Proposition 3.6, Proposition 3.8, Remark 3.9 and by using standard arguments based on the strong Markov property (cf. [53]) we may show that $v$ solves the free-boundary problem

$$
\left\{\begin{array}{cl}
\left(\partial_{t}+\mathcal{L}-\bar{\mu}\right) v(t, y)=-R_{c}(y) & \text { for } \hat{y}_{+}(t)<y<\hat{y}_{-}(t), t \in[0, T) \\
\left(\partial_{t}+\mathcal{L}-\bar{\mu}\right) v(t, y) \leq-R_{c}(y) & \text { for } y>\hat{y}_{+}(t), t \in[0, T) \\
\left(\partial_{t}+\mathcal{L}-\bar{\mu}\right) v(t, y) \geq-R_{c}(y) & \text { for } y<\hat{y}_{-}(t), t \in[0, T) \\
\frac{c_{-}}{f_{C}} \leq v(t, y) \leq \frac{c_{+}}{f_{C}} & \text { in }[0, T] \times(0, \infty) \\
v\left(t, \hat{y}_{ \pm}(t)\right)=\frac{c_{ \pm}}{f_{C}} & t \in[0, T) \\
v(T, y)=\frac{c_{-}}{f_{C}} & y>0
\end{array}\right.
$$

with $\mathcal{L} f:=\frac{1}{2} \sigma_{C}^{2} y^{2} f^{\prime \prime}+\hat{\mu}_{C} y f^{\prime}$ for $f \in C_{b}^{2}((0, \infty))$, and $\hat{\mu}_{C}:=-\mu_{C}+\frac{1}{2} \sigma_{C}^{2}$. Moreover $v \in C^{1,2}$ inside the continuation region $\mathcal{C}$.

Proposition 3.10. Under Assumptions 2.1 and 3.3 one has

1. $\hat{y}_{+}(t)$ and $\hat{y}_{-}(t)$ are decreasing;

2. $\hat{y}_{+}(t)$ is left-continuous and $\hat{y}_{-}(t)$ is right-continuous;

3. $0<\hat{y}_{+}(t) \leq R_{c}^{-1}\left(\frac{\bar{\mu} c_{+}}{f_{C}}\right)$, for $t \in[0, T)$;

4. $\lim _{t \uparrow T} \hat{y}_{+}(t)=: \hat{y}_{+}(T)=0$;

5. $0<R_{c}^{-1}\left(\frac{\bar{\mu} c_{-}}{f_{C}}\right) \leq \hat{y}_{-}(t)<+\infty$, for $t \in[0, T)$;

6. $\lim _{t \uparrow T} \hat{y}_{-}(t)=: \hat{y}_{-}(T-)=R_{c}^{-1}\left(\frac{\bar{\mu} c_{-}}{f_{C}}\right)$.

Proof. 1. We borrow arguments from [37]. Fix $t \in[0, T]$ and take $s \in[0, T-t]$. Then for any $\epsilon>0$ one has

$$
v\left(t+s, \hat{y}_{+}(t)+\epsilon\right) \leq v\left(t, \hat{y}_{+}(t)+\epsilon\right)<\frac{c_{+}}{f_{C}},
$$

being $v(\cdot, y)$ decreasing by Proposition 3.7 and since $\hat{y}_{+}(t)+\epsilon \in \mathcal{C}_{t}$. Then $\hat{y}_{+}(t)+\epsilon \in \mathcal{C}_{t+s}$ and therefore

$$
\hat{y}_{+}(t)+\epsilon \geq \hat{y}_{+}(t+s),
$$

i.e. $\hat{y}_{+}(t)$ is decreasing. Similar arguments apply to show that $\hat{y}_{-}(t)$ is decreasing as well. 
2. Fix $t \in[0, T]$ and notice that for every $\epsilon \in[0, t]$ we have $\hat{y}_{+}(t) \leq \hat{y}_{+}(t-\epsilon)$. It follows that $\hat{y}_{+}(t) \leq \lim _{\epsilon \downarrow 0} \hat{y}_{+}(t-\epsilon)=: \hat{y}_{+}(t-)$, which exists since $\hat{y}_{+}(\cdot)$ is monotone. Consider now the family $\left(t-\epsilon, \hat{y}_{+}(t-\epsilon)\right)_{\epsilon>0} \in \mathcal{S}_{+}$; one has $\left(t-\epsilon, \hat{y}_{+}(t-\epsilon)\right) \rightarrow\left(t, \hat{y}_{+}(t-)\right)$ as $\epsilon \downarrow 0$ and $\left(t, \hat{y}_{+}(t-)\right) \in \mathcal{S}_{+}$, since $\mathcal{S}_{+}$is closed. Recalling $\mathcal{S}_{+, t}$ of Proposition 3.8, one has $\hat{y}_{+}(t-) \leq \hat{y}_{+}(t)$ and thus $\hat{y}_{+}(t-)=\hat{y}_{+}(t)$. Right-continuity of $\hat{y}_{-}(\cdot)$ follows by similar arguments.

3. To show that $\hat{y}_{+}(t)>0$ for any $t<T$ we argue by contradiction and we assume that $\hat{y}_{+}(t)=0$ for some $t \in[0, T)$. From monotonicity of $\hat{y}_{+}(\cdot)$ we have $\hat{y}_{+}(t+s)=0$ for every $s \in[0, T-t)$. Take now $y \in \mathcal{C}_{t}$ and notice that $y C^{0}(s)>0, s \in[0, T-t)$. It follows that $\sigma^{*}=T-t$,

$$
\begin{aligned}
v(t, y) & =\sup _{\tau \in[0, T-t]} \tilde{\mathbb{E}}\left\{\frac{c_{-}}{f_{C}} e^{-\bar{\mu} \tau}+\int_{0}^{\tau \wedge(T-t)} e^{-\bar{\mu} s} R_{c}\left(y C^{0}(s)\right) d s\right\} \\
& >\tilde{\mathbb{E}}\left\{\int_{0}^{T-t} e^{-\bar{\mu} s} R_{c}\left(y C^{0}(s)\right) d s\right\},
\end{aligned}
$$

and

$$
v(t, y)-\frac{c_{+}}{f_{C}}>\tilde{\mathbb{E}}\left\{\int_{0}^{T-t} e^{-\bar{\mu} s} R_{c}\left(y C^{0}(s)\right) d s\right\}-\frac{c_{+}}{f_{C}} .
$$

The right-hand side of (3.21) may be taken strictly positive by monotone convergence and Inada conditions (cf. Assumption 2.1) for $y$ sufficiently small. Such a contradiction proves that $\hat{y}_{+}(t)>0$ for any $t<T$.

Given that $\mathcal{S}_{+, t}$ is connected (cf. Proposition 3.8), $\hat{y}_{+}$is positive and decreasing, then $\mathcal{S}_{+}$is connected, with non-empty interior int $\mathcal{S}_{+}$. Taking $v=c_{+} / f_{C}$ in the third equation of (3.20) one has int $\mathcal{S}_{+} \subseteq\left\{(t, y) \in[0, T) \times(0, \infty): R_{c}(y) \geq \frac{\bar{\mu} c_{+}}{f_{C}}\right\}$. Therefore, setting $\bar{y}_{+}:=R_{c}^{-1}\left(\frac{\bar{\mu} c_{+}}{f_{C}}\right)$ one finds $\hat{y}_{+}(t) \leq \bar{y}_{+}$for all $t \in[0, T)$.

4. If $\hat{y}_{+}(T)>0$ then we would have $\lim _{y \downarrow \hat{y}_{+}(T)} v(T, y)=\frac{c_{-}}{f_{C}}$ and $\lim _{t \uparrow T} v\left(t, \hat{y}_{+}(t)\right)=\frac{c_{+}}{f_{C}}$, but this contradicts the continuity of $v$ on $[0, T] \times(0, \infty)$ (cf. Theorem 3.4).

5. We shall first show that $\hat{y}_{-}(t)<+\infty$. To accomplsh that we introduce an auxiliary optimal stopping problem with free boundary $b(t)$ such that $\hat{y}_{-}(t) \leq b(t)$ and $b(t)<+\infty$. Notice that for any $(t, y) \in[0, T] \times(0, \infty)$ one has

$$
v(t, y) \leq \tilde{v}(t, y)
$$

with

$$
\tilde{v}(t, y):=\sup _{\tau \in[0, T-t]} \tilde{\mathbb{E}}\left\{\frac{c_{-}}{f_{C}} e^{-\bar{\mu} \tau}+\int_{0}^{\tau} e^{-\bar{\mu} s} R_{c}\left(y C^{0}(s)\right) d s\right\}
$$

by simply taking $\sigma=T-t$ in (3.8). It is not hard to see that $\tilde{v}(t, y) \geq \frac{c_{-}}{f_{C}}$ for any $(t, y) \in$ $[0, T] \times(0, \infty), y \mapsto \tilde{v}(t, y)$ is decreasing for any $t \in[0, T]$ due to the concavity of $R, t \mapsto \tilde{v}(t, y)$ is decreasing and continuous for any $y \in(0, \infty)$, and $y \mapsto \tilde{v}(t, y)$ is continuous uniformly in $t$. Then $(t, y) \mapsto \tilde{v}(t, y)$ is continuous on $[0, T] \times(0, \infty)$ and the stopping time

$$
\tilde{\tau}^{*}(t, y):=\inf \left\{s \in[0, T-t): \tilde{v}\left(t+s, y C^{0}(s)\right) \leq \frac{c_{-}}{f_{C}}\right\} \wedge(T-t)
$$

is optimal (cf. for instance [53]). Moreover, there exists a unique monotone decreasing free boundary

$$
b(t):=\inf \left\{y \in(0, \infty): \tilde{v}(t, y)=\frac{c_{-}}{f_{C}}\right\}, t<T,
$$

such that the continuation region $\tilde{C}$ is the open set

$$
\tilde{C}:=\{y \in(0, \infty): y<b(t), t<T\} .
$$


Since $v(t, y) \leq \tilde{v}(t, y)$, then it is not hard to show that $\hat{y}_{-}(t) \leq b(t)$. We will now prove that $b(t)<\infty$ for all $t \in[0, T]$ adapting arguments by [51]. Assume there exists $0<t_{o}<T$ such that $b\left(t_{o}\right)=+\infty$, then $\tilde{\tau}^{*}(0, y) \geq t_{o}$ for any $y>0$ and

$$
\tilde{v}(0, y)=\frac{c_{-}}{f_{C}}+\tilde{\mathbb{E}}\left\{\int_{0}^{\tilde{\tau}^{*}(0, y)} e^{-\bar{\mu} s}\left(R_{c}\left(y C^{0}(s)\right)-\frac{\bar{\mu} c_{-}}{f_{C}}\right) d s\right\}
$$

since for any stopping time $\tau \in[0, T]$

$$
e^{-\bar{\mu} \tau} \frac{c_{-}}{f_{C}}=\frac{c_{-}}{f_{C}}-\int_{0}^{\tau} e^{-\bar{\mu} s} \frac{\bar{\mu} c_{-}}{f_{C}} d s
$$

Fix $\epsilon>0$, set $\bar{y}_{-}:=R_{c}^{-1}\left(\frac{\bar{\mu} c_{-}}{f_{C}}\right)$ and define the stopping time

$$
\tau_{\bar{y}_{-}}^{\epsilon}(0, y):=\inf \left\{s \in[0, T): y C^{0}(s) \leq \bar{y}_{-}+\epsilon\right\} \wedge T \text {. }
$$

Observe that there exists $q_{\epsilon}>0$ such that $R_{c}(y)-\frac{\bar{\mu} c_{-}}{f_{C}}<-q_{\epsilon}$ for all $y \geq \bar{y}_{-}+\epsilon$, by (3.20). From now on we write $\tilde{\tau}^{*} \equiv \tilde{\tau}^{*}(0, y)$ and $\tau_{\bar{y}_{-}}^{\epsilon} \equiv \tau_{\bar{y}_{-}}^{\epsilon}(0, y)$ to simplify notation. We then have

$$
\begin{aligned}
\tilde{v}(0, y)-\frac{c_{-}}{f_{C}}= & \tilde{\mathbb{E}}\left\{\mathbb{1}_{\left\{\tilde{\tau}^{*} \leq \tau_{\bar{y}_{-}}\right\}} \int_{0}^{\tilde{\tau}^{*}} e^{-\bar{\mu} s}\left(R_{c}\left(y C^{0}(s)\right)-\frac{\bar{\mu} c_{-}}{f_{C}}\right) d s\right\} \\
& +\tilde{\mathbb{E}}\left\{\mathbb{1}_{\left\{\tilde{\tau}^{*}>\tau_{\bar{y}_{-}}\right\}} \int_{0}^{\tilde{\tau}^{*}} e^{-\bar{\mu} s}\left(R_{c}\left(y C^{0}(s)\right)-\frac{\bar{\mu} c_{-}}{f_{C}}\right) d s\right\} \\
\leq & -q_{\epsilon} \tilde{\mathbb{E}}\left\{\tilde{\tau}^{*} \mathbb{1}_{\left\{\tilde{\tau}^{*} \leq \tau_{\bar{y}_{-}}\right\}}\right\} \\
& +\tilde{\mathbb{P}}\left(\tilde{\tau}^{*}>\tau_{\bar{y}_{-}}\right)^{\frac{1}{2} \tilde{\mathbb{E}}}\left\{\left|\int_{0}^{T} e^{-\bar{\mu} s}\left(R_{c}\left(y C^{0}(s)\right)-\frac{\bar{\mu} c_{-}}{f_{C}}\right) d s\right|^{2}\right\}^{\frac{1}{2}} \\
\leq & -q_{\epsilon} t_{o} \tilde{\mathbb{P}}\left(\tilde{\tau}^{*} \leq \tau_{\bar{y}_{-}}^{\epsilon}\right)+c(y) \tilde{\mathbb{P}}\left(\tilde{\tau}^{*}>\tau_{\bar{y}_{-}}\right)^{\frac{1}{2}},
\end{aligned}
$$

where we have used Hölder inequality and set $c(y):=\tilde{\mathbb{E}}\left\{\left|\int_{0}^{T} e^{-\bar{\mu} s}\left[R_{c}\left(y C^{0}(s)\right)-\frac{\bar{\mu} c_{-}}{f_{C}}\right] d s\right|^{2}\right\}^{\frac{1}{2}}<$ $\infty$ (which is bounded by some positive constant $\bar{\kappa}$ as $y \uparrow \infty$ by Lemma A.1 in Appendix). If now

$$
\lim _{y \uparrow \infty} \tilde{\mathbb{P}}\left(\tilde{\tau}^{*}(0, y)>\tau_{\bar{y}_{-}}^{\epsilon}(0, y)\right)=0,
$$

then we have $\tilde{v}(0, y)-\frac{c_{-}}{f_{C}}<0$ for $y$ sufficiently large, thus reaching a contradiction.

To verify that, take now $y>\bar{y}_{-}+\epsilon$ and notice that

$$
\begin{aligned}
& \left\{\tau_{\bar{y}_{-}}^{\epsilon}(0, y)<\tilde{\tau}^{*}(0, y)\right\} \subseteq\left\{\inf _{0 \leq s \leq T} y C^{0}(s) \leq \bar{y}_{-}+\epsilon\right\} \\
& =\left\{\inf _{0 \leq s \leq T} \sigma_{C} \tilde{W}(s)+\hat{\mu}_{C} s \leq-\ln \left(\frac{y}{\bar{y}_{-}+\epsilon}\right)\right\} \subseteq\left\{\sup _{0 \leq s \leq T}\left|\sigma_{C} \tilde{W}(s)+\hat{\mu}_{C} s\right| \geq \ln \left(\frac{y}{\bar{y}_{-}+\epsilon}\right)\right\},
\end{aligned}
$$

with $\hat{\mu}_{C}$ as in (3.7). Then we obtain

$$
\begin{aligned}
\tilde{\mathbb{P}}\left(\tilde{\tau}^{*}(0, y)>\tau_{\bar{y}_{-}}^{\epsilon}(0, y)\right) & \leq \tilde{\mathbb{P}}\left(\sup _{0 \leq s \leq T}\left|\sigma_{C} \tilde{W}(s)+\hat{\mu}_{C} s\right| \geq \ln \left(\frac{y}{\bar{y}_{-}+\epsilon}\right)\right) \\
& \leq\left[\ln \left(\frac{y}{\bar{y}_{-}+\epsilon}\right)\right]^{-1} \tilde{\mathbb{E}}\left\{\sup _{0 \leq s \leq T}\left|\sigma_{C} \tilde{W}(s)+\hat{\mu}_{C} s\right|\right\} \leq C_{T}\left[\ln \left(\frac{y}{\bar{y}_{-}+\epsilon}\right)\right]^{-1},
\end{aligned}
$$

where we used Markov inequality and standard estimates on the solutions of stochastic differential equations (cf. [29], Chapter 5). It follows (3.26) and that $b\left(t_{o}\right)<+\infty$. 
It remains now to exclude the case $t_{o}=0$ as well. Assume $b(0)=+\infty$, take $\delta>0,0<t<\delta$ and define

$$
\tilde{v}_{\delta}(t, y):=\sup _{\tau \in[0, T+\delta-t]} \tilde{\mathbb{E}}\left\{\frac{c_{-}}{f_{C}} e^{-\bar{\mu} \tau}+\int_{0}^{\tau} e^{-\bar{\mu} s} R_{c}\left(y C^{0}(s)\right) d s\right\}
$$

Hence $\tilde{v}_{\delta}(t, y) \geq \tilde{v}(t, y)$ and $\tilde{v}_{\delta}(t+\delta, y)=\tilde{v}(t, y)$. If we now denote by $b_{\delta}$ the free-boundary of problem (3.27), we easily find $b(0)=b_{\delta}(\delta)$. We may thus repeat same arguments as those employed in the case $t_{o}>0$ to obtain a contradiction and conclude that $b(0)<+\infty$. Finally, we may proceed as in the second part of the proof of 3 . to show that $\hat{y}_{-}(t) \geq \bar{y}_{-}$for all $t \in[0, T]$.

6. Define $\hat{b}_{-}(t):=\hat{y}_{-}(t)-\bar{y}_{-}$, with $\bar{y}_{-}:=R_{c}^{-1}\left(\frac{\bar{\mu} c_{-}}{f_{C}}\right)$. This curve is nonnegative thanks to 5. and $\hat{b}_{-}(t)<\hat{y}_{-}(t)$ for all $t \leq T$; that is, $\left(t, \hat{b}_{-}(t)\right) \in \mathcal{S}_{+} \cup \mathcal{C}$ and $v\left(t, \hat{b}_{-}(t)\right)>\frac{c_{-}}{f_{C}}$ for all $t \leq T$. If now $\hat{b}_{-}(T-)>0$ then $\lim _{y \uparrow \hat{b}_{-}(T-)} v(T, y)=\frac{c_{-}}{f_{C}}$ and $\lim _{t \uparrow T} v\left(t, \hat{b}_{-}(t)\right)>\frac{c_{-}}{f_{C}}$, but this is not possible being $v(t, y)$ continuous on $[0, T] \times(0, \infty)$ by Theorem 3.4. It then follows $\hat{b}_{-}(T-)=0$, i.e. $\hat{y}_{-}(T-)=\bar{y}_{-}$.

Theorem 3.11. The free-boundaries $t \mapsto \hat{y}_{+}(t)$ and $t \mapsto \hat{y}_{-}(t)$ are continuous on $[0, T]$.

Proof. A proof of continuity by standard use of Newton-Leibnitz formula (cf. [53] for a list of examples) seems rather hard to implement for the lower free-boundary $\hat{y}_{+}$. In fact, inequalities that one would normally try to use cannot be obtained in that case. For this reason we abandon that approach and proceed via arguments inspired by PDE theory.

1. We start by considering the upper free-boundary, $\hat{y}_{-}(t)$, which is right-continuous (cf. Proposition 3.10). Let us argue by contradiction and assume that there exists $t_{o} \in(0, T)$ where a discontinuity of $\hat{y}_{-}(\cdot)$ occurs; that is, $t_{o}$ is such that $\hat{y}_{-}\left(t_{o}-\right)>\hat{y}_{-}\left(t_{o}\right)$. Fix $t^{\prime} \in\left(0, t_{o}\right), y_{1}$ and $y_{2}$ such that $\hat{y}_{-}\left(t_{o}\right)<y_{1}<y_{2}<\hat{y}_{-}\left(t_{o}-\right)$ and define a domain $\mathcal{R} \subset \mathcal{C}$ by $\mathcal{R}:=\left(t^{\prime}, t_{o}\right) \times\left(y_{1}, y_{2}\right)$. Its parabolic boundary $\partial_{P} \mathcal{R}$ is clearly formed by the horizontal lines $\left[t^{\prime}, t_{o}\right) \times\left\{y_{i}\right\}, i=1,2$ and by the vertical line $\left\{t_{o}\right\} \times\left[y_{1}, y_{2}\right]$. From the first equation in (3.20) and the definition of $\mathcal{R}$ we obtain that $v$ (uniquely) solves the Dirichlet-Cauchy problem

$$
\begin{array}{ll}
\left(\partial_{t}+\mathcal{L}-\bar{\mu}\right) u(t, y)=-R_{c}(y) & \text { in } \mathcal{R} \\
u\left(t, y_{1}\right)=v\left(t, y_{1}\right) & t \in\left[t^{\prime}, t_{0}\right) \\
u\left(t, y_{2}\right)=v\left(t, y_{2}\right) & t \in\left[t^{\prime}, t_{0}\right) \\
u\left(t_{0}, y\right)=\frac{c_{-}}{f_{C}} & y \in\left[y_{1}, y_{2}\right] .
\end{array}
$$

We denote by $C_{c}^{\infty}\left(\left[y_{1}, y_{2}\right]\right)$ the set of functions with infinitely many continuous derivatives and compact support in $\left[y_{1}, y_{2}\right]$. Take $\psi \geq 0$ arbitrary in $C_{c}^{\infty}\left(\left[y_{1}, y_{2}\right]\right)$ and such that $\int_{y_{1}}^{y_{2}} \psi(y) d y=1$. Multiply the first equation in (3.28) (with $v$ instead of $u$ ) by $\psi$ and integrate over $\left[y_{1}, y_{2}\right]$. It gives

$$
\int_{y_{1}}^{y_{2}} \partial_{t} v(t, y) \psi(y) d y=-\int_{y_{1}}^{y_{2}}\left[(\mathcal{L}-\bar{\mu}) v(t, y)+R_{c}(y)\right] \psi(y) d y \quad \text { for all } t \in\left[t^{\prime}, t_{o}\right) .
$$

We now integrate by parts twice the term on the right hand side of (3.29) and obtain

$$
\int_{y_{1}}^{y_{2}} \partial_{t} v(t, y) \psi(y) d y=-\int_{y_{1}}^{y_{2}}\left[v(t, y)\left(\mathcal{L}^{*}-\bar{\mu}\right)+R_{c}(y)\right] \psi(y) d y \quad \text { for all } t \in\left[t^{\prime}, t_{o}\right),
$$


where $\mathcal{L}^{*}$ is the adjoint of $\mathcal{L}$ and, in this particular case, it reads

$$
\mathcal{L}^{*} \psi(y):=\frac{1}{2} \sigma_{C}^{2} y^{2} \psi^{\prime \prime}(y)+\left(2 \sigma_{C}^{2}-\hat{\mu}_{C}\right) y \psi^{\prime}(y)+\left(\sigma_{C}^{2}-\hat{\mu}_{C}\right) \psi(y) .
$$

Recall that $\partial_{t} v$ is negative by 3 . of Proposition 3.7. Take the limit as $t \rightarrow t_{o}$ in (3.30), use dominated convergence, Theorem 3.4 and the last equation in (3.28) to obtain

$$
\begin{aligned}
0 & \geq \lim _{t \uparrow t_{o}} \int_{y_{1}}^{y_{2}} \partial_{t} v(t, y) \psi(y) d y=-\int_{y_{1}}^{y_{2}}\left[v\left(t_{o}, y\right)\left(\mathcal{L}^{*}-\bar{\mu}\right)+R_{c}(y)\right] \psi(y) d y \\
& =-\int_{y_{1}}^{y_{2}}\left[\frac{c_{-}}{f_{C}}\left(\mathcal{L}^{*}-\bar{\mu}\right)+R_{c}(y)\right] \psi(y) d y=-\int_{y_{1}}^{y_{2}}\left[R_{c}(y)-\frac{\bar{\mu} c_{-}}{f_{C}}\right] \psi(y) d y .
\end{aligned}
$$

Notice that $y \mapsto R_{c}(y)-\frac{\bar{\mu} c_{-}}{f_{C}}$ is continuous and strictly negative for $y \in\left[y_{1}, y_{2}\right]$, by 5 . of Proposition 3.10 as $y_{1}>\bar{y}_{-}$and $R_{c}(\cdot)$ is strictly decreasing. Hence, there exists a positive constant $\ell:=\ell\left(y_{1}, y_{2}\right)$ such that $\sup _{y \in\left[y_{1}, y_{2}\right]}\left[R_{c}(y)-\frac{\bar{\mu} c_{-}}{f_{C}}\right] \leq-\ell$ and from the last term of $(3.32)$ we find

$$
0 \geq-\int_{y_{1}}^{y_{2}}\left[R_{c}(y)-\frac{\bar{\mu} c_{-}}{f_{C}}\right] \psi(y) d y \geq \ell \int_{y_{1}}^{y_{2}} \psi(y) d y=\ell>0,
$$

by using that $\int_{y_{1}}^{y_{2}} \psi(y) d y=1$. Therefore, we reach a contradiction and $\hat{y}_{-}\left(t_{o}-\right)=\hat{y}_{-}\left(t_{o}\right)$.

2 . We will now prove continuity of the lower boundary $\hat{y}_{+}(\cdot)$. Again we argue by contradiction and assume that there exists $t_{o} \in(0, T)$ where a discontinuity of $\hat{y}_{+}(\cdot)$ occurs. Then $t_{o}$ is such that $\hat{y}_{+}\left(t_{o}\right)>\hat{y}_{+}\left(t_{o}+\right)$. As before we define an open bounded domain $\mathcal{R} \subset \mathcal{C}$ with parabolic boundary $\partial_{P} \mathcal{R}$ formed by the horizontal lines $\left[t_{o}, t^{\prime}\right) \times\left\{y_{i}\right\}, i=1,2$ and by the vertical line $\left\{t^{\prime}\right\} \times\left[y_{1}, y_{2}\right]$ with $y_{1}$ and $y_{2}$ such that $\hat{y}_{+}\left(t_{o}+\right)<y_{1}<y_{2}<\hat{y}_{+}\left(t_{o}\right)$ and arbitrary $t^{\prime} \in\left(t_{o}, T\right)$. We have that $u:=\frac{c_{+}}{f_{C}}-v$ solves

$$
\left(\partial_{t}+\mathcal{L}-\bar{\mu}\right) u(t, y)=R_{c}(y)-\frac{\bar{\mu} c_{+}}{f_{C}}, \quad(t, y) \in \mathcal{R},
$$

by (3.20) and additionally $u\left(t_{o}, y\right) \equiv 0$ for $y \in\left[y_{1}, y_{2}\right]$. Regularity of $R_{c}$ and of the coefficients in $\mathcal{L}$ imply that $u_{y y y}$ and $u_{t y}$ exist and are continuous in $\mathcal{R}$ (cf. [27], Theorem 10, Chapter 3). Differentiating (3.34) with respect to $y$ and defining $\bar{u}:=u_{y}$ we easily obtain

$$
\bar{u}_{t}(t, y)+\frac{1}{2} \sigma_{C}^{2} y^{2} \bar{u}_{y y}(t, y)+\left(\sigma_{C}^{2}+\hat{\mu}_{C}\right) y \bar{u}_{y}(t, y)+\left(\hat{\mu}_{C}-\bar{\mu}\right) \bar{u}(t, y)=R_{c c}(y)<0, \quad(t, y) \in \mathcal{R},
$$

as $R$ is strictly concave. It will be useful in what follows to define the second order differential operator

$$
\mathcal{G} f(y):=\frac{1}{2} \sigma_{C}^{2} y^{2} f^{\prime \prime}(y)+\left(\sigma_{C}^{2}+\hat{\mu}_{C}\right) y f^{\prime}(y)+\left(\hat{\mu}_{C}-\bar{\mu}\right) f(y) \quad \text { for } f \in C_{b}^{2}(\mathbb{R}) .
$$

Again we consider a test function $\psi \in C_{c}^{\infty}\left(\left[y_{1}, y_{2}\right]\right)$ such that $\psi \geq 0$ and $\int_{y_{1}}^{y_{2}} \psi(y) d y=1$. We define a function $F_{\psi}:\left(t_{o}, T\right) \rightarrow \mathbb{R}$ by

$$
F_{\psi}(t):=\int_{y_{1}}^{y_{2}} \bar{u}_{t}(t, y) \psi(y) d y, \quad t \in\left(t_{o}, T\right) .
$$

Now, denoting by $\mathcal{G}^{*}$ the formal adjoint of $\mathcal{G}$ in (3.36), (3.35) gives

$$
\begin{aligned}
F_{\psi}(t) & =\int_{y_{1}}^{y_{2}}\left[R_{c c}(y)-\mathcal{G} \bar{u}(t, y)\right] \psi(y) d y=\int_{y_{1}}^{y_{2}}\left[R_{c c}(y) \psi(y)-\bar{u}(t, y) \mathcal{G}^{*} \psi(y)\right] d y \\
& =\int_{y_{1}}^{y_{2}}\left[R_{c c}(y) \psi(y)+u(t, y) \frac{\partial}{\partial y}\left(\mathcal{G}^{*} \psi\right)(y)\right] d y .
\end{aligned}
$$


The map $t \mapsto F_{\psi}(t)$ is clearly continuous on $\left(t_{o}, T\right)$, its right-limit at $t_{o}$ is well defined thanks to dominated convergence and it is equal to

$$
F_{\psi}\left(t_{o}+\right):=\lim _{t \downarrow t_{o}} F_{\psi}(t)=\int_{y_{1}}^{y_{2}} R_{c c}(y) \psi(y) d y
$$

by recalling that $u\left(t_{o}, y\right) \equiv 0$ for $y \in\left[y_{1}, y_{2}\right]$. From strict concavity of $R$, there exists $\ell>0$ such that $R_{c c}(y)<-\ell$ in $\left[y_{1}, y_{2}\right]$ and hence $F_{\psi}\left(t_{o}+\right)<-\ell$. It follows that there exists $\epsilon>0$ such that $F_{\psi}(t)<-\ell / 2$ for all $t \in\left(t_{o}, t_{o}+\epsilon\right]$ by continuity of $F_{\psi}$. Now, take $0<\delta<\epsilon$ arbitrary, then (3.37) and Fubini's theorem give

$$
\begin{aligned}
-\frac{\ell}{2}(\epsilon-\delta) & >\int_{\delta}^{\epsilon} F_{\psi}\left(t_{o}+s\right) d s=\int_{y_{1}}^{y_{2}}\left[\bar{u}\left(t_{o}+\epsilon, y\right)-\bar{u}\left(t_{o}+\delta, y\right)\right] \psi(y) d y \\
& =\int_{y_{1}}^{y_{2}} u_{y}\left(t_{o}+\epsilon, y\right) \psi(y) d y+\int_{y_{1}}^{y_{2}} u\left(t_{o}+\delta, y\right) \psi^{\prime}(y) d y
\end{aligned}
$$

Taking limits as $\delta \rightarrow 0$ we obtain

$$
-\frac{\ell}{2} \epsilon \geq \int_{y_{1}}^{y_{2}} u_{y}\left(t_{o}+\epsilon, y\right) \psi(y) d y=-\int_{y_{1}}^{y_{2}} v_{y}\left(t_{o}+\epsilon, y\right) \psi(y) d y \geq 0
$$

since $y \mapsto v(t, y)$ is decreasing (cf. Proposition 3.7). Therefore we reach a contradiction and $\hat{y}_{+}$ must be continuous on $(0, T)$.

3. It remains only to prove continuity at $T$. Since $\hat{y}_{+}$is left-continuous (cf. 2. of Proposition $3.10)$ then it is continuous on $[0, T]$. On the other hand, $\hat{y}_{-}$is right-continuous and decreasing with $\hat{y}_{-}(T-)=R_{c}^{-1}\left(\frac{\bar{\mu} c_{+}}{f_{C}}\right)$ (see 6 . of Proposition 3.10). Then, it must be continuous on $[0, T]$ since $\hat{y}_{-}(t) \geq R_{c}^{-1}\left(\frac{\bar{\mu} c_{+}}{f_{C}}\right)$ for all $t \in[0, T]$.

Recall that $R \in C^{2}((0, \infty))$ and it is strictly concave. We now make the following

Assumption 3.12. For any $y_{o}>0$ there exists $\delta_{o}:=\delta_{o}\left(y_{o}\right)$ such that

$$
\tilde{\mathbb{E}}\left\{\int_{0}^{T} e^{-\bar{\mu} s} \inf _{\left\{y:\left|y-y_{o}\right| \leq \delta_{o}\right\}} R_{c c}\left(y C^{0}(s)\right) d s\right\}>-\infty .
$$

It is easy to see that Assumption 3.12 is fulfilled by Cobb-Douglas and logarithmic production function.

Proposition 3.13. Let Assumption 2.1, 3.3 and 3.12 hold. Then the smooth-fit property holds at the free boundaries $\hat{y}_{+}$and $\hat{y}_{-}$. That is,

$$
\begin{array}{ll}
v_{y}\left(t, \hat{y}_{-}(t)-\right)=0, & t \in[0, T), \\
v_{y}\left(t, \hat{y}_{+}(t)+\right)=0, & t \in[0, T) .
\end{array}
$$

Proof. We start by proving (3.43). Fix $\epsilon>0$ and $t_{o} \in[0, T)$ and let $\left(\sigma_{-\epsilon}^{*}, \tau_{-\epsilon}^{*}\right)$ be optimal in $v\left(t_{o}, \hat{y}_{-}\left(t_{o}\right)-\epsilon\right)$ in the sense of (3.11). Since the free-boundary $\hat{y}_{-}$is monotone decreasing, it is not hard to show that

$$
\lim _{\epsilon \rightarrow 0} \tau_{-\epsilon}^{*}=0, \quad \mathbb{P} \text {-a.s. }
$$

by the law of iterated logarithm at zero for Brownian motion. 
Take $\sigma^{*}:=\sigma^{*}\left(t_{o}, \hat{y}_{-}\left(t_{o}\right)\right)$ as in (3.11) and adopt the sub-optimal stopping strategy $\left(\sigma^{*}, \tau_{-\epsilon}^{*}\right)$ in both the optimization problems with value functions $v\left(t_{o}, \hat{y}_{-}\left(t_{o}\right)\right)$ and $v\left(t_{o}, \hat{y}_{-}\left(t_{o}\right)-\epsilon\right)$. Then, using that $y \mapsto v(t, y)$ is decreasing (cf. Proposition 3.7) we obtain

$$
\begin{aligned}
0 & \leq v\left(t_{o}, \hat{y}_{-}\left(t_{o}\right)-\epsilon\right)-v\left(t_{o}, \hat{y}_{-}\left(t_{o}\right)\right) \\
& \leq \tilde{\mathbb{E}}\left\{\int_{0}^{\sigma^{*} \wedge \tau_{-\epsilon}^{*}} e^{-\bar{\mu} s}\left[R_{c}\left(\left(\hat{y}_{-}\left(t_{o}\right)-\epsilon\right) C^{0}(s)\right)-R_{c}\left(\hat{y}_{-}\left(t_{o}\right) C^{0}(s)\right)\right] d s\right\}
\end{aligned}
$$

and an application of the mean value theorem gives

$$
0 \leq v\left(t_{o}, \hat{y}_{-}\left(t_{o}\right)-\epsilon\right)-v\left(t_{o}, \hat{y}_{-}\left(t_{o}\right)\right) \leq-\epsilon \tilde{\mathbb{E}}\left\{\int_{0}^{\sigma^{*} \wedge \tau_{-\epsilon}^{*}} e^{-\bar{\mu} s} R_{c c}\left(\xi_{\epsilon} C^{0}(s)\right) d s\right\}
$$

for some $\xi_{\epsilon} \in\left[\hat{y}_{-}\left(t_{o}\right)-\epsilon, \hat{y}_{-}\left(t_{o}\right)\right]$. Thanks to Assumption 3.12, fixed $y_{o}:=\hat{y}_{-}\left(t_{o}\right)$, we can always find $\delta_{o}>\epsilon$, such that (3.42) holds. Then, dividing (3.47) by $\epsilon$ we have

$$
0 \leq \frac{v\left(t_{o}, y_{o}-\epsilon\right)-v\left(t_{o}, y_{o}\right)}{\epsilon} \leq \tilde{\mathbb{E}}\left\{-\int_{0}^{\sigma^{*} \wedge \tau_{-\epsilon}^{*}} e^{-\bar{\mu} s} \inf _{y \in\left[y_{o}-\delta_{o}, y_{o}\right]} R_{c c}\left(y C^{0}(s)\right) d s\right\}
$$

for all $\epsilon<\delta_{o}$. The family $\left(Z_{\epsilon}\right)_{\epsilon \in\left(0, \delta_{o}\right)}$ defined by

$$
Z_{\epsilon}:=-\int_{0}^{\sigma^{*} \wedge \tau_{-\epsilon}^{*}} e^{-\bar{\mu} s} \inf _{y \in\left[y_{o}-\delta_{o}, y_{o}\right]} R_{c c}\left(y C^{0}(s)\right) d s
$$

is uniformly bounded from above by

$$
H:=-\int_{0}^{T} e^{-\bar{\mu} s} \inf _{y \in\left[y_{o}-\delta_{o}, y_{o}\right]} R_{c c}\left(y C^{0}(s)\right) d s
$$

and $H$ is $\tilde{\mathbb{P}}$-integrable by Assumption 3.12. Therefore, Fatou's lemma, (3.45) and (3.48) imply

$$
0 \leq \lim _{\epsilon \rightarrow 0} \frac{v\left(t_{o}, \hat{y}_{-}\left(t_{o}\right)-\epsilon\right)-v\left(t_{o}, \hat{y}_{-}\left(t_{o}\right)\right)}{\epsilon} \leq 0
$$

and hence (3.43).

We now prove (3.44). Unfortunately arguments as in (3.43) seem not to be applicable in this context. In fact, fixed $t_{o} \in[0, T)$, if we define by $\left(\sigma_{+\epsilon}^{*}, \tau_{+\epsilon}^{*}\right)$ optimal stopping times for $v\left(t_{o}, \hat{y}_{+}\left(t_{o}\right)+\right.$ $\epsilon)$, then we would like to have $\lim _{\epsilon \rightarrow 0} \sigma_{+\epsilon}^{*}=0$ a.s. However, that does not seem obvious since $\hat{y}_{+}$is only proved to be continuous and decreasing. In fact, roughly speaking, we cannot exclude the case that $\hat{y}_{+}^{\prime}\left(t_{o}\right)=-\infty$ at countably many points $t_{o}$. To avoid this difficulty, we shall adopt a different argument inspired by [54].

Let $h$ be a $C^{2}$ solution on $(0, \infty)$ of the second-order ordinary differential equation $\mathcal{L} h(y)=$ $R_{c}(y)$. Fix $\left(t_{o}, y\right) \in[0, T) \times(0, \infty)$ and let $\rho$ be a stopping time. Then, from $i$ ) of Proposition 3.6 one has

$$
\begin{aligned}
v\left(t_{o}, y\right) & \leq \tilde{\mathbb{E}}\left\{e^{-\bar{\mu}\left(\rho \wedge \tau^{*}\right)} v\left(t_{o}+\rho \wedge \tau^{*}, y C^{0}\left(\rho \wedge \tau^{*}\right)\right)+\int_{0}^{\rho \wedge \tau^{*}} e^{-\bar{\mu} s} R_{c}\left(y C^{0}(s)\right) d s\right\} \\
& \leq \tilde{\mathbb{E}}\left\{v\left(t_{o}+\rho \wedge \tau^{*}, y C^{0}\left(\rho \wedge \tau^{*}\right)\right)+\int_{0}^{\rho} R_{c}\left(y C^{0}(s)\right) d s\right\} \\
& =\tilde{\mathbb{E}}\left\{v\left(t_{o}+\rho \wedge \tau^{*}, y C^{0}\left(\rho \wedge \tau^{*}\right)\right)+h\left(y C^{0}(\rho)\right)\right\}-h(y),
\end{aligned}
$$

by Dynkin formula and the definition of $h$. Therefore

$$
v\left(t_{o}, y\right)+h(y) \leq \tilde{\mathbb{E}}\left\{v\left(t_{o}+\rho \wedge \tau^{*}, y C^{0}\left(\rho \wedge \tau^{*}\right)\right)+h\left(y C^{0}(\rho)\right)\right\} .
$$


For any $\alpha>0$ we define the hitting time $\tau_{\alpha}:=\inf \left\{s \geq 0: y C^{0}(s)=\alpha\right\}$. Take $0<c<y<d<\bar{y}_{-}$ and set $\rho:=\tau_{c} \wedge \tau_{d}$. Then $\rho \wedge \tau^{*}=\rho \wedge\left(T-t_{o}\right)$ and (3.53) becomes

$$
\begin{aligned}
& v\left(t_{o}, y\right)+h(y) \leq \tilde{\mathbb{E}}\{v\left.\left(t_{o}+\rho, y C^{0}(\rho)\right) \mathbb{1}_{\left\{\rho<T-t_{o}\right\}}\right\} \\
& \quad+\frac{c_{-}}{f_{C}} \tilde{\mathbb{E}}\left\{\mathbb{1}_{\left\{\rho \geq T-t_{o}\right\}}\right\}+h(c) \tilde{\mathbb{P}}\left(\tau_{c}<\tau_{d}\right)+h(d) \tilde{\mathbb{P}}\left(\tau_{d}<\tau_{c}\right) \\
&=\tilde{\mathbb{E}}\left\{v\left(t_{o}+\tau_{c}, c\right) \mathbb{1}_{\left\{\rho<T-t_{o}\right\}} \mathbb{1}_{\left\{\tau_{c}<\tau_{d}\right\}}+v\left(t_{o}+\tau_{d}, d\right) \mathbb{1}_{\left\{\rho<T-t_{o}\right\}} \mathbb{1}_{\left\{\tau_{d}<\tau_{c}\right\}}\right\} \\
& \quad+\frac{c_{-}}{f_{C}} \tilde{\mathbb{E}}\left\{\mathbb{1}_{\left\{\rho \geq T-t_{o}\right\}}\right\}+h(c) \tilde{\mathbb{P}}\left(\tau_{c}<\tau_{d}\right)+h(d) \tilde{\mathbb{P}}\left(\tau_{d}<\tau_{c}\right) .
\end{aligned}
$$

Recall now that $t \mapsto v(t, y)$ is decreasing (cf. Proposition 3.7), that $v\left(t_{o}, y\right) \geq \frac{c_{-}}{f_{C}}$ for any $y \in(0, \infty)$, and that $v(T, c)=v(T, d)=c_{-} / f_{C}$. Hence (3.54) implies

$$
\begin{gathered}
v\left(t_{o}, y\right)+h(y) \leq v\left(t_{o}, c\right) \tilde{\mathbb{E}}\left\{\mathbb{1}_{\left\{\rho<T-t_{o}\right\}} \mathbb{1}_{\left\{\tau_{c}<\tau_{d}\right\}}\right\}+v\left(t_{o}, d\right) \tilde{\mathbb{E}}\left\{\mathbb{1}_{\left\{\rho<T-t_{o}\right\}} \mathbb{1}_{\left\{\tau_{d}<\tau_{c}\right\}}\right\} \\
\quad+\frac{c_{-}}{f_{C}} \tilde{\mathbb{E}}\left\{\mathbb{1}_{\left\{\rho \geq T-t_{o}\right\}}\right\}+h(c) \tilde{\mathbb{P}}\left(\tau_{c}<\tau_{d}\right)+h(d) \tilde{\mathbb{P}}\left(\tau_{d}<\tau_{c}\right) \\
\leq v\left(t_{o}, c\right) \tilde{\mathbb{E}}\left\{\mathbb{1}_{\left\{\rho<T-t_{o}\right\}} \mathbb{1}_{\left\{\tau_{c}<\tau_{d}\right\}}\right\}+v\left(t_{o}, d\right) \tilde{\mathbb{E}}\left\{\mathbb{1}_{\left\{\rho<T-t_{o}\right\}} \mathbb{1}_{\left\{\tau_{d}<\tau_{c}\right\}}\right\} \\
\quad+v\left(t_{o}, c\right) \tilde{\mathbb{E}}\left\{\mathbb{1}_{\left\{\rho \geq T-t_{o}\right\}} \mathbb{1}_{\left\{\tau_{c}<\tau_{d}\right\}}\right\}+v\left(t_{o}, d\right) \tilde{\mathbb{E}}\left\{\mathbb{1}_{\left\{\rho \geq T-t_{o}\right\}} \mathbb{1}_{\left\{\tau_{d}<\tau_{c}\right\}}\right\} \\
\quad+h(c) \tilde{\mathbb{P}}\left(\tau_{c}<\tau_{d}\right)+h(d) \tilde{\mathbb{P}}\left(\tau_{d}<\tau_{c}\right) \\
=\left[v\left(t_{o}, c\right)+h(c)\right] \tilde{\mathbb{P}}\left(\tau_{c}<\tau_{d}\right)+\left[v\left(t_{o}, d\right)+h(d)\right] \tilde{\mathbb{P}}\left(\tau_{d}<\tau_{c}\right) \\
=\left[v\left(t_{o}, c\right)+h(c)\right] \frac{S(d)-S(y)}{S(d)-S(c)}+\left[v\left(t_{o}, d\right)+h(d)\right] \frac{S(y)-S(c)}{S(d)-S(c)},
\end{gathered}
$$

where $S$ is the scale function (see, e.g., [42], Chapter 5 ) of $C^{0}$. It follows that, for fixed $t_{o} \in[0, T$ ), the function $y \mapsto u\left(t_{o}, y\right)$, defined by $u\left(t_{o}, y\right):=v\left(t_{o}, y\right)+h(y)$, is $S$-convex (see, e.g., [58], p. 546). Therefore

$$
y \mapsto \frac{u\left(t_{o}, y\right)-u\left(t_{o}, x\right)}{S(y)-S(x)}
$$

is increasing on $[c, d]$, for every $x \in(c, d)$.

Notice now that the scale function $S$ of a geometric Brownian motion admits first order derivative at any $y \in(0, \infty)$ and recall that $h \in C^{2}((0, \infty))$. Then, for arbitrary but fixed $t_{o} \in[0, T)$, we can apply arguments as in [54], Theorem 2.3, and obtain

$$
u_{y}\left(t_{o}, \hat{y}_{+}\left(t_{o}\right)+\right)=h^{\prime}\left(\hat{y}_{+}\left(t_{o}\right)\right) .
$$

Hence

$$
v_{y}\left(t_{o}, \hat{y}_{+}\left(t_{o}\right)+\right)=0 \quad t_{o} \in[0, T),
$$

by definition of $u$.

In the next Theorem we will find non-linear integral equations that characterise the free boundaries and the value function $v$ of our zero-sum optimal stopping game.

Theorem 3.14. Under Assumption 2.1, 3.3 and 3.12, the value function $v$ of problem (3.8) has the following representation

$$
\begin{aligned}
v(t, y)= & e^{-\bar{\mu}(T-t)} \frac{c_{-}}{f_{C}}+\int_{0}^{T-t} e^{-\bar{\mu} s} \tilde{\mathbb{E}}\left\{R_{C}\left(y C^{0}(s)\right) \mathbb{1}_{\left\{\hat{y}_{+}(t+s)<y C^{0}(s)<\hat{y}_{-}(t+s)\right\}}\right\} d s \\
& +\frac{\bar{\mu}}{f_{C}} \int_{0}^{T-t} e^{-\bar{\mu} s}\left[c_{+} \tilde{\mathbb{P}}\left(y C^{0}(s)<\hat{y}_{+}(t+s)\right)+c_{-} \tilde{\mathbb{P}}\left(y C^{0}(s)>\hat{y}_{-}(t+s)\right)\right] d s
\end{aligned}
$$


where $\hat{y}_{+}$and $\hat{y}_{-}$are continuous, decreasing curves solving the coupled integral equations

$$
\begin{aligned}
& \frac{c_{-}}{f_{C}}=e^{-\bar{\mu}(T-t)} \frac{c_{-}}{f_{C}}+\int_{0}^{T-t} e^{-\bar{\mu} s} \tilde{\mathbb{E}}\left\{R_{c}\left(\hat{y}_{-}(t) C^{0}(s)\right) \mathbb{1}_{\left\{\hat{y}_{+}(t+s)<\hat{y}_{-}(t) C^{0}(s)<\hat{y}_{-}(t+s)\right\}}\right\} d s \\
& +\frac{\bar{\mu}}{f_{C}} \int_{0}^{T-t} e^{-\bar{\mu} s}\left[c_{+} \tilde{\mathbb{P}}\left(\hat{y}_{-}(t) C^{0}(s)<\hat{y}_{+}(t+s)\right)+c_{-} \tilde{\mathbb{P}}\left(\hat{y}_{-}(t) C^{0}(s)>\hat{y}_{-}(t+s)\right)\right] d s
\end{aligned}
$$

and

$$
\begin{aligned}
& \frac{c_{+}}{f_{C}}=e^{-\bar{\mu}(T-t)} \frac{c_{-}}{f_{C}}+\int_{0}^{T-t} e^{-\bar{\mu} s} \tilde{\mathbb{E}}\left\{R_{c}\left(\hat{y}_{+}(t) C^{0}(s)\right) \mathbb{1}_{\left\{\hat{y}_{+}(t+s)<\hat{y}_{+}(t) C^{0}(s)<\hat{y}_{-}(t+s)\right\}}\right\} d s \\
& +\frac{\bar{\mu}}{f_{C}} \int_{0}^{T-t} e^{-\bar{\mu} s}\left[c_{+} \tilde{\mathbb{P}}\left(\hat{y}_{+}(t) C^{0}(s)<\hat{y}_{+}(t+s)\right)+c_{-} \tilde{\mathbb{P}}\left(\hat{y}_{+}(t) C^{0}(s)>\hat{y}_{-}(t+s)\right)\right] d s,
\end{aligned}
$$

with boundary conditions

$$
\hat{y}_{-}(T)=R_{c}^{-1}\left(\frac{\bar{\mu} c_{-}}{f_{C}}\right) \quad \& \quad \hat{y}_{+}(T)=0
$$

and such that

$$
R_{c}^{-1}\left(\frac{\bar{\mu} c_{-}}{f_{C}}\right) \leq \hat{y}_{-}(t)<+\infty \quad \& \quad 0 \leq \hat{y}_{+}(t) \leq R_{c}^{-1}\left(\frac{\bar{\mu} c_{+}}{f_{C}}\right) \quad \text { for all } t \in[0, T] .
$$

Proof. We aim to apply local time-space formula by [52], Theorem 3.1. In order to do so we will verify that $v$ fulfils suitable sufficient conditions. That is, for $\eta>0$ arbitrary but fixed

$$
\begin{aligned}
& \left(\partial_{t}+\mathcal{L}-\bar{\mu}\right) v \quad \text { is bounded on any compact } K \text { in }[0, T-\eta] \times(0,+\infty) \\
& t \mapsto v_{y}\left(t, \hat{y}_{ \pm}(t) \pm\right)=0 \quad \text { is continuous on }[0, T-\eta] \\
& t \mapsto v\left(t, \hat{y}_{ \pm}(t) \pm\right) \quad \text { is of bounded variation on }[0, T-\eta] .
\end{aligned}
$$

Conditions (3.61) and (3.62) follow from (3.20) and the smooth-fit property (cf. Proposition 3.13).

To verify (3.63) we need a bit more work. There exists $\delta_{\eta}:=\delta(\eta)>0$ such that $\hat{y}_{+}(t)>\delta_{\eta}$ for all $t \in[0, T-\eta]$, by 3. of Proposition 3.10 and Theorem 3.11. Also, there exist: $L_{\eta}:=L\left(\delta_{\eta}\right)>0$ such that $\left|v_{y}(t, y)\right| \leq L_{\eta}$ for all $y \in\left[\hat{y}_{+}(t)-\delta_{\eta}, \hat{y}_{+}(t)+\delta_{\eta}\right], t \in[0, T-\eta]$ by $(3.62)$ and $R_{\eta}:=R\left(\delta_{\eta}\right)>0$ such that $R_{c}(y) \leq R_{\eta}$ on $y \geq \hat{y}_{+}(T-\eta)-\delta_{\eta}$. From these bounds, 2. of Proposition 3.7, and the first equation in (3.20) we find

$$
\frac{\sigma_{C}^{2}}{2} y^{2} v_{y y} \geq-R_{\eta}-\left|\hat{\mu}_{C}\right| L_{\eta} y+\bar{\mu} \frac{c_{-}}{f_{C}}, \quad y \in\left[\hat{y}_{+}(t)-\delta_{\eta}, \hat{y}_{+}(t)+\delta_{\eta}\right], \quad t \in[0, T-\eta] .
$$

Now, divide both sides of (3.64) by $\frac{\sigma_{C}^{2}}{2} y^{2}$ to obtain

$$
v_{y y} \geq-\left(\frac{2 R_{\eta}}{\sigma_{C}^{2}}\right) \frac{1}{y^{2}}-\left(\frac{2\left|\hat{\mu}_{C}\right| L_{\eta}}{\sigma_{C}^{2}}\right) \frac{1}{y}, \quad y \in\left[\hat{y}_{+}(t)-\delta_{\eta}, \hat{y}_{+}(t)+\delta_{\eta}\right], \quad t \in[0, T-\eta],
$$

and recall that $\hat{y}_{+}(T-\eta) \leq \hat{y}_{+}(t)$ for $t \in[0, T-\eta]$. If we define

$$
F(y):=-\int_{\hat{y}_{+}(T-\eta)-\delta_{\eta}}^{y} \int_{\hat{y}_{+}(T-\eta)-\delta_{\eta}}^{z}\left[\left(\frac{2 R_{\eta}}{\sigma_{C}^{2}}\right) \frac{1}{r^{2}}+\left(\frac{2\left|\hat{\mu}_{C}\right| L_{\eta}}{\sigma_{C}^{2}}\right) \frac{1}{r}\right] d r d z,
$$


then $y \mapsto \Lambda(t, y):=[v-F](t, y)$ is convex on $\left[\hat{y}_{+}(t), \hat{y}_{+}(t)+\delta_{\eta}\right]$ and on $\left[\hat{y}_{+}(t)-\delta_{\eta}, \hat{y}_{+}(t)\right]$ for all $t \in[0, T-\eta]$. Also, it is easily verified that $t \mapsto \Lambda_{y}\left(t, \hat{y}_{ \pm}(t) \pm\right)$ is continuous on $[0, T-\eta]$ by (3.62) and (3.66). From (3.61) and (3.66) we obtain that $\partial_{t} \Lambda+\mathcal{L} \Lambda-\bar{\mu} \Lambda$ is bounded on any compact $K \subset[0, T-\eta] \times(0,+\infty)$. It follows that $t \mapsto \Lambda\left(t, \hat{y}_{ \pm}(t) \pm\right)$ is of bounded variation on $[0, T-\eta]$, by [52], Remark 3.2 (see in particular eqs. (3.35)-(3.36) therein). Therefore (3.63) holds as $t \mapsto F\left(\hat{y}_{ \pm}(t) \pm\right)$ is of bounded variation and hence $v$ has to be such as well.

The local time-space formula may now be employed on $[0, T-\eta] \times(0,+\infty)$. For any $(t, y) \in$ $[0, T-\eta] \times(0,+\infty)$ and arbitrary $s \leq T-\eta-t$, we have

$$
\begin{array}{rl}
e^{-\bar{\mu} s} & v\left(t+s, y C^{0}(s)\right) \\
= & v(t, y)+\int_{0}^{s} e^{-\bar{\mu} u}\left(\partial_{t} v+\mathcal{L} v-\bar{\mu} v\right)\left(t+u, y C^{0}(u)\right) \mathbb{1}_{\left\{\hat{y}_{+}(t+u)<y C^{0}(u)<\hat{y}_{-}(t+u)\right\}} d u \\
& -\frac{\bar{\mu}}{f_{C}} \int_{0}^{s} e^{-\bar{\mu} u}\left[c_{+} \mathbb{1}_{\left\{y C^{0}(u)<\hat{y}_{+}(t+u)\right\}}+c_{-} \mathbb{1}_{\left\{y C^{0}(u)>\hat{y}_{-}(t+u)\right\}}\right] d u+M(s),
\end{array}
$$

by (3.43) and (3.44) and with $M:=\{M(s), s \in[0, T-\eta-t]\}$ a local martingale. We can take expectations in (3.67) and use standard localization arguments to cancel the local martingale term. Then, setting $s=T-\eta-t$ we obtain

$$
\begin{aligned}
& v(t, y)=\tilde{\mathbb{E}}\left\{e^{-\bar{\mu}(T-t-\eta)} v\left(T-\eta, y C^{0}(T-t-\eta)\right)\right\} \\
& +\int_{0}^{T-t-\eta} e^{-\bar{\mu} u \tilde{\mathbb{E}}}\left\{R_{c}\left(y C^{0}(u)\right) \mathbb{1}_{\left\{\hat{y}_{+}(t+u)<y C^{0}(u)<\hat{y}_{-}(t+u)\right\}}\right\} d u \\
& +\frac{\bar{\mu}}{f_{C}} \int_{0}^{T-t-\eta} e^{-\bar{\mu} u}\left[c_{+} \tilde{\mathbb{P}}\left(y C^{0}(u)<\hat{y}_{+}(t+u)\right)+c_{-} \tilde{\mathbb{P}}\left(y C^{0}(u)>\hat{y}_{-}(t+u)\right)\right] d u
\end{aligned}
$$

by (3.20) and after rearranging terms. Since (3.68) holds for any $\eta>0$, in the limit as $\eta \downarrow 0$ we find (3.56) by dominated convergence and continuity of $v$.

If we now take $y=\hat{y}_{+}(t)$ (or $y=\hat{y}_{-}(t)$ ) in both sides of (3.56) we easily obtain (3.58) (or (3.57)) by recalling that $v\left(t, \hat{y}_{ \pm}(t)\right)=c_{ \pm} / f_{C}$.

It is now natural to ask whether the couple $\left(\hat{y}_{+}, \hat{y}_{-}\right)$is the unique solution of problem (3.57)(3.60). In many optimal stopping problems it is possible to show that the free-boundary, denoted for instance by $b(t)$, is in fact the unique solution of a (single) non-linear integral equation of Volterra type similar to (3.57) and (3.58) (see for instance [50]). The proof goes as follows: one assumes that another solution $c(t)$ exists and associates to that a suitable function, often denoted by $U^{c}$; then martingale arguments and properties of $U^{c}$ lead to a number of contradictions thus implying uniqueness. From a careful reading of such proof one evinces that it is crucial to show that the value function $V$ of a sup (inf) problem is always larger (smaller) than $U^{c}$.

In our zero-sum optimal stopping game a further complication arises from the fact that $v$ is a saddle point. Assuming that $\left(\alpha_{+}, \alpha_{-}\right)$is another solution of (3.57)-(3.60) and trying to argue as in [50], we define a function $u_{\alpha}:[0, T] \times(0, \infty) \mapsto \mathbb{R}$ by

$$
\begin{aligned}
u_{\alpha}(t, y):= & e^{-\bar{\mu}(T-t)} \frac{c_{-}}{f_{C}}+\int_{0}^{T-t} e^{-\bar{\mu} s} \tilde{\mathbb{E}}\left\{R_{c}\left(y C^{0}(s)\right) \mathbb{1}_{\left\{\alpha_{+}(t+s)<y C^{0}(s)<\alpha_{-}(t+s)\right\}}\right\} d s \\
& +\frac{\bar{\mu}}{f_{C}} \int_{0}^{T-t} e^{-\bar{\mu} s}\left[c_{+} \tilde{\mathbb{P}}\left(y C^{0}(s)<\alpha_{+}(t+s)\right)+c_{-} \tilde{\mathbb{P}}\left(y C^{0}(s)>\alpha_{-}(t+s)\right)\right] d s .
\end{aligned}
$$

It seems rather hard to prove that $u_{\alpha}$ of (3.69) is either larger or smaller than $v$. However, this issue may be overcome by further restricting the set of couples $\left(\alpha_{+}, \alpha_{-}\right)$solving (3.57)-(3.60) to those which also guarantee $c_{-} / f_{C} \leq u_{\alpha} \leq c_{+} / f_{C}$. This is to some extent equivalent to proving the existence of a unique triple $\left(v, \hat{y}_{+}, \hat{y}_{-}\right)$solving the free-boundary problem (3.20). 
Theorem 3.15. The couple $\left(\hat{y}_{+}(t), \hat{y}_{-}(t)\right)$ is the unique solution of (3.57) and (3.58) in the class of continuous, decreasing functions such that (3.59), (3.60) hold and such that $u_{\alpha}$ in (3.69) (with $\alpha_{+} \equiv \hat{y}_{+}$and $\alpha_{-} \equiv \hat{y}_{-}$) satisfies

$$
\frac{c_{-}}{f_{C}} \leq u_{\alpha}(t, y) \leq \frac{c_{+}}{f_{C}} \quad \text { for all }(t, y) \in[0, T] \times(0,+\infty) .
$$

Proof. Set $Y^{y}(s):=y C^{0}(s)$, under $\tilde{\mathbb{P}}$ to simplify notation. Assume there exist two continuous functions $\alpha_{-}$and $\alpha_{+}$solving (3.57)-(3.60) and such that $u_{\alpha}$ as in (3.69) fulfils (3.70). The map $(t, y) \mapsto u_{\alpha}(t, y)$ is continuous and $u_{\alpha}\left(t, \alpha_{ \pm}(t)\right)=c_{ \pm} / f_{C}$ for $t \in[0, T)$, by (3.57) and (3.58). It is not hard to verify that the process $U_{\alpha}^{t, y}:=\left\{U_{\alpha}^{t, y}(s), s \in[0, T-t]\right\}$, defined by

$$
\begin{aligned}
U_{\alpha}^{t, y}(s):=e^{-\bar{\mu} s} u_{\alpha}\left(t+s, Y^{y}(s)\right) & +\int_{0}^{s} e^{-\bar{\mu} u} R_{c}\left(Y^{y}(u)\right) \mathbb{1}_{\left\{\alpha_{+}(t+u)<Y^{y}(u)<\alpha_{-}(t+u)\right\}} d u \\
& +\frac{\bar{\mu}}{f_{C}} \int_{0}^{s} e^{-\bar{\mu} u}\left[c_{+} \mathbb{1}_{\left\{Y^{y}(u)<\alpha_{+}(t+u)\right\}}+c_{-} \mathbb{1}_{\left\{Y^{y}(u)>\alpha_{-}(t+u)\right\}}\right] d u
\end{aligned}
$$

is a $\tilde{\mathbb{P}}$-martingale.

Take $t \in[0, T)$ arbitrary and $y>\alpha_{-}(t)$. Define the stopping time

$$
\tau_{\alpha_{-}}(t, y):=\inf \left\{s \in[0, T-t): Y^{y}(s) \leq \alpha_{-}(t+s)\right\} \wedge(T-t)
$$

and for simplicity set $\tau_{\alpha_{-}}:=\tau_{\alpha_{-}}(t, y)$. From the martingale property of $U_{\alpha}^{t, y}$ in (3.71) we easily find

$$
u_{\alpha}(t, y)=\tilde{\mathbb{E}}\left\{e^{-\bar{\mu} \tau_{\alpha_{-}}} u_{\alpha}\left(t+\tau_{\alpha_{-}}, Y^{y}\left(\tau_{\alpha_{-}}\right)\right)+\bar{\mu} \frac{c_{-}}{f_{C}} \int_{0}^{\tau_{\alpha_{-}}} e^{-\bar{\mu} s} d s\right\}
$$

Continuity of the process $Y^{y}$ implies that $Y^{y}\left(\tau_{\alpha_{-}}\right)=\alpha_{-}\left(t+\tau_{\alpha_{-}}\right)$on the set $\left\{\tau_{\alpha_{-}}<T-t\right\}$. From (3.69), (3.72) and the continuity of $u_{\alpha}$ we observe that $u_{\alpha}\left(t+\tau_{\alpha_{-}}, Y^{y}\left(\tau_{\alpha_{-}}\right)\right)=c_{-} / f_{C}, \tilde{\mathbb{P}}-$ a.s., therefore

$$
u_{\alpha}(t, y)=\tilde{\mathbb{E}}\left\{e^{-\bar{\mu} \tau_{\alpha_{-}}} \frac{c_{-}}{f_{C}}+\bar{\mu} \frac{c_{-}}{f_{C}} \int_{0}^{\tau_{\alpha_{-}}} e^{-\bar{\mu} s} d s\right\}=\frac{c_{-}}{f_{C}} \quad \text { for all } y>\alpha_{-}(t) \text { and } t \in[0, T) .
$$

Similarly, consider $y<\alpha_{+}(t)$ for a given $t \in[0, T)$, define the stopping time

$$
\tau_{\alpha_{+}}(t, y):=\inf \left\{s \in[0, T-t): Y^{y}(s) \geq \alpha_{+}(t+s)\right\} \wedge(T-t) .
$$

and as usual set $\tau_{\alpha_{+}}:=\tau_{\alpha_{+}}(t, y)$, to simplify notation. We may now use same arguments as in (3.73) to obtain

$$
u_{\alpha}(t, y)=\tilde{\mathbb{E}}\left\{e^{-\bar{\mu} \tau_{\alpha_{+}}} u_{\alpha}\left(t+\tau_{\alpha_{+}}, Y^{y}\left(\tau_{\alpha_{+}}\right)\right)+\bar{\mu} \frac{c_{+}}{f_{C}} \int_{0}^{\tau_{\alpha_{+}}} e^{-\bar{\mu} s} d s\right\} .
$$

Note that on the set $\left\{\tau_{\alpha_{+}}<T-t\right\}$ one has $Y^{y}\left(\tau_{\alpha_{+}}\right)=\alpha_{+}\left(t+\tau_{\alpha_{+}}\right)$, by continuity of $Y^{y}$ and $\alpha_{+}$. On the other hand, $\left\{\tau_{\alpha_{+}}=T-t\right\} \subset\left\{Y^{y}(T-t)=0\right\}$, since $\alpha_{+}$is continuous and $\alpha_{+}(T)=0$; however, $\left\{Y^{y}(T-t)=0\right\}$ is a $\tilde{\mathbb{P}}$-null set and hence we conclude that $u_{\alpha}\left(t+\tau_{\alpha_{+}}, Y^{y}\left(\tau_{\alpha_{+}}\right)\right)=c_{+} / f_{C}$, $\tilde{\mathbb{P}}$-a.s. Then, from $(3.76)$ we obtain

$$
u_{\alpha}(t, y)=\tilde{\mathbb{E}}\left\{e^{-\bar{\mu} \tau_{\alpha_{+}}} \frac{c_{+}}{f_{C}}+\bar{\mu} \frac{c_{+}}{f_{C}} \int_{0}^{\tau_{\alpha_{+}}} e^{-\bar{\mu} s} d s\right\}=\frac{c_{+}}{f_{C}} \quad \text { for all } y<\alpha_{+}(t) \text { and } t \in[0, T) .
$$


We shall now prove that $\alpha_{+} \equiv \hat{y}_{+}$and $\alpha_{-} \equiv \hat{y}_{-}$. Initially we show that

$$
\alpha_{+}(t) \leq \hat{y}_{+}(t) \quad \text { and } \quad \alpha_{-}(t) \geq \hat{y}_{-}(t) \quad \text { for all } t \in[0, T) .
$$

Full details are only provided for the first of (3.78) as the ones for the second can be obtained analogously. Assume that there exists $t_{o} \in[0, T)$ such that $\hat{y}_{+}\left(t_{o}\right)<\alpha_{+}\left(t_{o}\right)$. Then, take $y_{o} \in$ $\left(\hat{y}_{+}\left(t_{o}\right), \alpha_{+}\left(t_{o}\right)\right)$ and define the stopping time

$$
\rho_{\alpha_{-}}\left(t_{o}, y_{o}\right):=\inf \left\{s \in\left[0, T-t_{o}\right): Y^{y_{o}}(s) \geq \alpha_{-}\left(t_{o}+s\right)\right\} \wedge\left(T-t_{o}\right) .
$$

Let $\sigma^{*}\left(t_{o}, y_{o}\right)$ be as in (3.11) (or equivalently as in (3.19)) and set $\rho_{\alpha_{-}}:=\rho_{\alpha_{-}}\left(t_{o}, y_{o}\right)$ and $\sigma^{*}:=$ $\sigma^{*}\left(t_{o}, y_{o}\right)$ for simplicity. From the martingale property of $U_{\alpha}^{t_{o}, y_{o}}$ in (3.71) we obtain

$$
\begin{aligned}
u_{\alpha}\left(t_{o}, y_{o}\right)=\tilde{\mathbb{E}}\{ & e^{-\bar{\mu} \sigma^{*} \wedge \rho_{\alpha_{-}}} u_{\alpha}\left(t_{o}+\sigma^{*} \wedge \rho_{\alpha_{-}}, Y^{y_{o}}\left(\sigma^{*} \wedge \rho_{\alpha_{-}}\right)\right) \\
& \left.+\int_{0}^{\sigma^{*} \wedge \rho_{\alpha_{-}}} e^{-\bar{\mu} s}\left[R_{c}\left(Y^{y_{o}}(s)\right) \mathbb{1}_{\left\{Y^{y_{o}}(s)>\alpha_{+}\left(t_{o}+s\right)\right\}}+\bar{\mu} \frac{c_{+}}{f_{C}} \mathbb{1}_{\left\{Y^{y_{o}}(s)<\alpha_{+}\left(t_{o}+s\right)\right\}}\right] d s\right\} .
\end{aligned}
$$

The first term in the expectation of (3.80) is such that

$$
u_{\alpha}\left(t+\sigma^{*} \wedge \rho_{\alpha_{-}}, Y^{y_{o}}\left(\sigma^{*} \wedge \rho_{\alpha_{-}}\right)\right) \leq \frac{c_{-}}{f_{C}} \mathbb{1}_{\left\{\rho_{\alpha_{-}} \leq \sigma^{*}\right\}} \mathbb{1}_{\left\{\sigma^{*}<T-t\right\}}+\frac{c_{+}}{f_{C}} \mathbb{1}_{\left\{\rho_{\left.\alpha_{-}>\sigma^{*}\right\}}\right.}+\frac{c_{-}}{f_{C}} \mathbb{1}_{\left\{\rho_{\alpha_{-}=\sigma^{*}=T-t}\right\}}
$$

by (3.69) and (3.70). Observe that all (continuous) sample paths starting from $y_{o}$ spend a strictly positive amount of time under the curve $\left\{\alpha_{+}\left(t_{o}+s\right), s \in\left[0, T-t_{o}\right)\right\}$ by continuity of $\alpha_{+}$. Moreover, from (3.60) we have

$$
\bar{\mu} \frac{c_{+}}{f_{C}}<R_{c}\left(Y^{y_{o}}(s)\right) \text { on the set }\left\{Y^{y_{o}}(s)<\alpha_{+}\left(t_{o}+s\right) \cdot\right\}
$$

Recall (3.9) and note that $\sigma^{*} \wedge \rho_{\alpha_{-}}(\omega)>0$ for $\tilde{\mathbb{P}}$-a.e. $\omega \in \Omega$, by continuity of $t \mapsto Y^{y_{o}}(t)$. Then, using (3.81) and (3.82) inside (3.80), we find

$$
\begin{aligned}
& u_{\alpha}\left(t_{o}, y_{o}\right)<\tilde{\mathbb{E}}\left\{e^{-\bar{\mu} \rho_{\alpha_{-}}} \frac{c_{-}}{f_{C}} \mathbb{1}_{\left\{\rho_{\alpha_{-}} \leq \sigma^{*}\right\}} \mathbb{1}_{\left\{\sigma^{*}<T-t_{o}\right\}}+e^{-\bar{\mu} \sigma^{*}} \frac{c_{+}}{f_{C}} \mathbb{1}_{\left\{\rho_{\alpha_{-}}>\sigma^{*}\right\}}\right. \\
& \left.+e^{-\bar{\mu}\left(T-t_{o}\right)} \frac{c_{-}}{f_{C}} \mathbb{1}_{\left\{\rho_{\alpha_{-}}=\sigma^{*}=T-t_{o}\right\}}+\int_{0}^{\sigma^{*} \wedge \rho_{\alpha_{-}}} e^{-\bar{\mu} s} R_{c}\left(Y^{y_{o}}(s)\right) d s\right\} \\
& =\Psi\left(t_{o}, y_{o} ; \sigma^{*}, \rho_{\alpha_{-}}\right) \text {. }
\end{aligned}
$$

It follows that $u_{\alpha}\left(t_{o}, y_{o}\right)<v\left(t_{o}, y_{o}\right)$. However, $u_{\alpha}\left(t_{o}, y\right)=c_{+} / f_{C}$ for all $y \in\left(0, \alpha_{+}(t)\right)$ by $(3.77)$ and hence $v\left(t_{o}, y_{o}\right)>c_{+} / f_{C}$. This is a contradiction as $\left(t_{o}, y_{o}\right) \in \mathcal{C}$. Similarly, one can find analogous contradiction by assuming that there exists $t_{o} \in[0, T)$ such that $\alpha_{-}\left(t_{o}\right)<\hat{y}_{-}\left(t_{o}\right)$.

We show now that it must in fact be $\alpha_{+} \equiv \hat{y}_{+}$and $\alpha_{-} \equiv \hat{y}_{-}$. Again, we provide full details only for $\alpha_{+}$as the other case follows by straightforward modifications. Assume that there exists $t_{o} \in[0, T)$ such that $\alpha_{+}\left(t_{o}\right)<\hat{y}_{+}\left(t_{o}\right)$. Take $y_{o} \in\left(\alpha_{+}\left(t_{o}\right), \hat{y}_{+}\left(t_{o}\right)\right)$, set $\tau^{*}\left(t_{o}, y_{o}\right)$ as in (3.11) and define

$$
\rho_{\alpha_{+}}\left(t_{o}, y_{o}\right):=\inf \left\{s \in\left[0, T-t_{o}\right): Y^{y_{o}}(s) \leq \alpha_{+}\left(t_{o}+s\right)\right\} \wedge\left(T-t_{o}\right) .
$$

Denote $\tau^{*}:=\tau^{*}\left(t_{o}, y_{o}\right)$ and $\rho_{\alpha_{+}}:=\rho_{\alpha_{+}}\left(t_{o}, y_{o}\right)$ for simplicity. We now set $s:=\tau^{*} \wedge \rho_{\alpha_{+}} \wedge\left(T-t_{o}-\eta\right)$ in (3.67), take the expectation on both sides, then pass to the limit as $\eta \rightarrow 0$ and rearrange terms 
to obtain

$$
\begin{aligned}
v\left(t_{o}, y_{o}\right)= & \tilde{\mathbb{E}}\left\{e^{-\bar{\mu} \tau^{*} \wedge \rho_{\alpha_{+}}} v\left(t_{o}+\tau^{*} \wedge \rho_{\alpha_{+}}, Y^{y_{o}}\left(\tau^{*} \wedge \rho_{\alpha_{+}}\right)\right)\right. \\
& \left.+\int_{0}^{\tau^{*} \wedge \rho_{\alpha_{+}}} e^{-\bar{\mu} s}\left[R_{c}\left(Y^{y_{o}}(s)\right) \mathbb{1}_{\left\{\hat{y}_{+}\left(t_{o}+s\right)<Y^{y_{o}}(s)\right\}}+\frac{\bar{\mu} c_{+}}{f_{C}} \mathbb{1}_{\left\{Y^{y_{o}}(s)<\hat{y}_{+}\left(t_{o}+s\right)\right\}}\right] d s\right\} .
\end{aligned}
$$

Since $\alpha_{+}(t) \leq \hat{y}_{+}(t)$ for $t \in[0, T)$ (cf. (3.78)), it is not hard to see that $v\left(t_{o}+\rho_{\alpha_{+}}, Y^{y_{o}}\left(\rho_{\alpha_{+}}\right)\right)=\frac{c_{+}}{f_{C}}$ on $\left\{\rho_{\alpha_{+}}<\tau^{*}\right\}$. Again we notice that $\tau^{*} \wedge \rho_{\alpha_{+}}(\omega)>0$ for $\tilde{\mathbb{P}}$-a.e. $\omega \in \Omega$, by continuity of the sample paths of $Y^{y_{o}}$ and that from (3.60)

$$
\bar{\mu} \frac{c_{+}}{f_{C}}<R_{c}\left(Y^{y_{o}}(s)\right) \text { on the set }\left\{Y^{y_{o}}(s)<\hat{y}_{+}\left(t_{o}+s\right)\right\} .
$$

Since all sample paths starting from $y_{o}$ spend a strictly positive amount of time below $\left\{\hat{y}_{+}\left(t_{o}+\right.\right.$ $\left.s), s \in\left[0, T-t_{o}\right)\right\}$ by continuity of $\hat{y}_{+}$, we obtain

$$
\begin{aligned}
v\left(t_{o}, y_{o}\right)<\tilde{\mathbb{E}}\left\{e^{-\bar{\mu} \tau^{*}} \frac{c_{-}}{f_{C}} \mathbb{1}_{\left\{\tau^{*} \leq \rho_{\alpha_{+}}\right\}} \mathbb{1}_{\left\{\rho_{\alpha_{+}}<T-t_{o}\right\}}+e^{-\bar{\mu} \rho_{\alpha_{+}}} \frac{c_{+}}{f_{C}} \mathbb{1}_{\left\{\tau^{*}>\rho_{\alpha_{+}}\right\}}\right. \\
\left.+e^{-\bar{\mu}\left(T-t_{o}\right)} \frac{c_{-}}{f_{C}} \mathbb{1}_{\left\{\rho_{\alpha_{+}}=\tau^{*}=T-t_{o}\right\}}+\int_{0}^{\tau^{*} \wedge \rho_{\alpha_{+}}} e^{-\bar{\mu} s} R_{c}\left(Y^{y_{o}}(s)\right) d s\right\},
\end{aligned}
$$

by $(3.86)$.

On the other hand, recalling (3.70), (3.78) and using the martingale property of $U_{\alpha}^{t_{o}, y_{o}}$ as in (3.80) we obtain

$$
\begin{aligned}
u_{\alpha}\left(t_{o}, y_{o}\right) \geq \tilde{\mathbb{E}}\{ & e^{-\bar{\mu} \tau^{*}} \frac{c_{-}}{f_{C}} \mathbb{1}_{\left\{\tau * \leq \rho_{\alpha_{+}}\right\}} \mathbb{1}_{\left\{\rho_{\alpha_{+}}<T-t_{o}\right\}}+e^{-\bar{\mu} \rho_{\alpha_{+}} \frac{c_{+}}{f_{C}} \mathbb{1}_{\left\{\tau^{*}>\rho_{\alpha_{+}}\right\}}} \\
& \left.+e^{-\bar{\mu}\left(T-t_{o}\right)} \frac{c_{-}}{f_{C}} \mathbb{1}_{\left\{\rho_{\alpha_{+}}=\tau^{*}=T-t_{o}\right\}}+\int_{0}^{\tau^{*} \wedge \rho_{\alpha_{+}}} e^{-\bar{\mu} s} R_{c}\left(Y^{y_{o}}(s)\right) d s\right\} .
\end{aligned}
$$

It follows from (3.87) and (3.88) that $u_{\alpha}\left(t_{o}, y_{o}\right)>v\left(t_{o}, y_{o}\right)$. However, $v\left(t_{o}, y_{o}\right)=c_{+} / f_{C}$ for $y_{o} \in$ $\left(\alpha_{+}\left(t_{o}\right), \hat{y}_{+}\left(t_{o}\right)\right)$ by $(3.20)$ and hence $u_{\alpha}\left(t_{o}, y_{o}\right)>c_{+} / f_{C}$, contradicting (3.70). Therefore $\alpha_{+} \equiv \hat{y}_{+}$ and by obvious extensions of arguments above one also finds $\alpha_{-} \equiv \hat{y}_{-}$.

\section{The Optimal Control Strategy}

In Theorem 2.4 we proved existence and uniqueness of the optimal control process $\nu^{*}$, but we provided no information about its nature. In this Section we characterize the optimal control in terms of the two free boundaries $\hat{y}_{+}$and $\hat{y}_{-}$(cf. (3.17) and (3.18)) of the zero-sum optimal stopping game (3.8). We shall see that the optimal investment-disinvestment strategy for problem (3.2) consists in keeping the optimally controlled diffusion $C^{y, \nu^{*}}$ inside the closure of the continuation region, with the optimal controls behaving as the local times of $C^{y, \nu^{*}}$ at $\hat{y}_{+}$and $\hat{y}_{-}$. To accomplish that we will rely on results in [14] on the pathwise construction of a process in a space-time region defined by two moving boundaries.

Recall (2.1) and (2.2) and introduce the following Skorokhod problem in a time-dependent interval.

Problem 4.1. Let $t \in[0, T]$ and $y>0$ be arbitrary but fixed. Given the two free boundaries $\hat{y}_{+}$ and $\hat{y}_{-}$of (3.17) and (3.18), respectively, we seek a left-continuous adapted process $C^{y, \bar{\nu}^{*}}$ and a 
process of bounded variation $\bar{\nu}^{*}=\bar{\nu}_{+}^{*}-\bar{\nu}_{-}^{*} \in \mathcal{S}$ such that

$$
\left\{\begin{array}{l}
C^{y, \bar{\nu}^{*}}(s)=C^{0}(s)\left[y+\bar{\nu}_{+}^{*}(s)-\bar{\nu}_{-}^{*}(s)\right], \quad s \in[0, T-t), \\
C^{y, \nu^{*}}(0)=y, \\
\hat{y}_{+}(t+s) \leq C^{y, \bar{\nu}^{*}}(s) \leq \hat{y}_{-}(t+s), \quad \text { a.e. } s \in[0, T-t], \\
\int_{0}^{T-t} \mathbb{1}_{\left\{C^{y, \bar{\nu}^{*}}(s)<\hat{y}_{-}(t+s)\right\}} d \bar{\nu}_{-}^{*}(s)=0, \\
\int_{0}^{T-t} \mathbb{1}_{\left\{C^{y, \bar{\nu}^{*}}(s)>\hat{y}_{+}(t+s)\right\}} d \bar{\nu}_{+}^{*}(s)=0
\end{array}\right.
$$

hold $\tilde{\mathbb{P}}_{-}$a.s. Moreover, if $y \in\left[\hat{y}_{+}(t), \hat{y}_{-}(t)\right]$ then $\bar{\nu}_{+}^{*}(\omega, \cdot)$ and $\bar{\nu}_{-}^{*}(\omega, \cdot)$ are continuous. When $y<$ $\hat{y}_{+}(t)$, then $\bar{\nu}_{+}^{*}(\omega, 0+)=\hat{y}_{+}(t)-y, \bar{\nu}_{-}^{*}(\omega, 0+)=0$ and $C^{y, \bar{\nu}^{*}}(\omega, 0+)=\hat{y}_{+}(t)$; when $y>\hat{y}_{-}(t)$, then $\bar{\nu}_{-}^{*}(\omega, 0+)=y-\hat{y}_{-}(t), \bar{\nu}_{+}^{*}(\omega, 0+)=0$ and $C^{y, \bar{\nu}^{*}}(\omega, 0+)=\hat{y}_{-}(t)$.

Proposition 4.2. There exists a unique solution of Problem 4.1 given by

$$
\left\{\begin{aligned}
& C^{y, \bar{\nu}^{*}}(s)= C^{0}(s)\left[y+\bar{\nu}^{*}(s)\right], \\
& \bar{\nu}^{*}(s+)=-\max \left\{\left[\left(y-\hat{y}_{-}(t)\right)^{+} \wedge \inf _{u \in[0, s]}\left(\frac{y C^{0}(u)-\hat{y}_{+}(t+u)}{C^{0}(u)}\right)\right],\right. \\
&\left.\sup _{r \in[0, s]}\left[\left(\frac{y C^{0}(r)-\hat{y}_{-}(t+r)}{C^{0}(r)}\right) \wedge \inf _{u \in[r, s]}\left(\frac{y C^{0}(u)-\hat{y}_{+}(t+u)}{C^{0}(u)}\right)\right]\right\},
\end{aligned}\right.
$$

for every $s \in[0, T-t)$.

Proof. Take $t \in[0, T]$ and $s \in[0, T-t]$ arbitrary but fixed and set

$$
\left\{\begin{array}{l}
\phi(s):=\frac{C^{y, \bar{\nu}^{*}}(s+)}{C^{0}(s)}, \quad \psi(s):=y, \\
\eta(s)=\eta_{\ell}(s)-\eta_{r}(s):=\bar{\nu}_{+}^{*}(s+)-\bar{\nu}_{-}^{*}(s+), \\
\ell(s):=\frac{\hat{y}_{+}(t+s)}{C^{0}(s)}, \quad r(s):=\frac{\hat{y}_{-}(t+s)}{C^{0}(s)} .
\end{array}\right.
$$

Notice that $\inf _{s \in[0, T-t]}[r(s)-\ell(s)]>0$, by Proposition 3.10. Hence, we can apply [14], Corollary 2.4 and Theorem 2.6 to obtain existence and uniqueness of the solution of Problem 4.1. Moreover, equations (2.6) and (2.7), give

$$
\begin{aligned}
C^{y, \bar{\nu}^{*}}(s+)= & y C^{0}(s)-C^{0}(s) \max \left\{\left[\left(y-\hat{y}_{-}(t)\right)^{+} \wedge \inf _{u \in[0, s]}\left(\frac{y C^{0}(u)-\hat{y}_{+}(t+u)}{C^{0}(u)}\right)\right],\right. \\
& \left.\sup _{r \in[0, s]}\left[\left(\frac{y C^{0}(r)-\hat{y}_{-}(t+r)}{C^{0}(r)}\right) \wedge \inf _{u \in[r, s]}\left(\frac{y C^{0}(u)-\hat{y}_{+}(t+u)}{C^{0}(u)}\right)\right]\right\},
\end{aligned}
$$

and the second equation in (4.2) follows from (2.3) since $C^{y, \bar{\nu}^{*}}(s+)=C^{0}(s)\left[y+\bar{\nu}^{*}(s+)\right]$. 
In order to prove that $C^{y, \bar{\nu}^{*}}$ is optimal for the control problem (3.2) it is useful to observe that $V_{t}, V_{y}, V_{y y}$ belong to $\mathbb{L}^{\infty}((0, T) \times(0, K))$, for arbitrary $K>0$, by (3.4), (3.20) and Proposition 3.13. Therefore, we can apply Itô's formula for semimartingales (cf. [57], Theorem 32, p. 79, among others) in the generalized sense of [8], Lemma 8.1 and Theorem 8.5, pp. 183-186, to obtain the following

Theorem 4.3. Let $\left(C^{y, \bar{\nu}^{*}}, \bar{\nu}^{*}\right)$ denote the unique solution of Problem 4.1. Then $C^{y, \nu^{*}}$ is the optimally controlled production capacity for problem (3.2) with $\nu^{*}:=\nu_{+}^{*}-\nu_{-}^{*}$ and

$$
\nu_{+}^{*}(s):=\int_{0}^{s} \frac{C^{0}(u)}{f_{C}} d \bar{\nu}_{+}^{*}(u), \quad \nu_{-}^{*}(s):=\int_{0}^{s} \frac{C^{0}(u)}{f_{C}} d \bar{\nu}_{-}^{*}(u),
$$

for every $s \in[0, T-t)$.

As expected (cf. [43], Theorem 3.1), the optimal time to invest (disinvest) coincides with the first time at which the uncontrolled diffusion hits the moving boundary $\hat{y}_{+}\left(\hat{y}_{-}\right)$.

\section{A Appendix}

\section{A.1 Proof of Theorem 3.4}

In this Section we show that the value function of the zero-sum optimal stopping game (3.8) is continuous on $[0, T] \times(0, \infty)$. To do so we shall follow arguments similar to those used in [61]. Note that analogous techniques had been also employed by Menaldi, among others, in an earlier paper (cf. [46]) where he studied an optimal stopping problem for degenerate diffusions. However,we cannot directly apply [61], Theorem 1 , since our marginal production function $R_{c}$ is not bounded as it is there required.

We now prove preliminary results and we introduce some new definitions that will be useful in the rest of this Appendix. Recall that

$$
\tilde{\mathbb{E}}\left\{\left(\frac{1}{C^{0}(s)}\right)^{\alpha}\right\}=e^{\left(\alpha \mu_{C}-\frac{1}{2} \alpha^{2} \sigma_{C}^{2}\right) s}
$$

for any $\alpha \geq 1$ and $s \in[0, T]$.

Lemma A.1. Under Assumption 2.1, for any $\alpha \geq 1$ one has

$$
\tilde{\mathbb{E}}\left\{\int_{0}^{T} R_{c}^{\alpha}\left(y C^{0}(s)\right) d s\right\} \leq \kappa\left(1+\frac{1}{y^{\alpha}}\right),
$$

where $\kappa>0$ is a suitable constant independent of $t$ and $y$.

Proof. Since $R(0)=0$ (cf. Assumption 2.1), for any $y>0$ we have $R_{c}(y) \leq y^{-1} R(y)$, by concavity of $R$. Also, Inada conditions imply that there exist $\kappa_{1}>0$ and $\kappa_{2}>0$ such that $R(y) \leq \kappa_{1}+\kappa_{2} y$ for all $y \in(0, \infty)$. Hence we have

$$
\begin{aligned}
\tilde{\mathbb{E}}\left\{\int_{0}^{T} R_{c}^{\alpha}\left(y C^{0}(s)\right) d s\right\} & \leq \tilde{\mathbb{E}}\left\{\int_{0}^{T}\left(\frac{1}{y C^{0}(s)}\right)^{\alpha}\left[\kappa_{1}+\kappa_{2} y C^{0}(s)\right]^{\alpha} d s\right\} \\
& \leq \mathbb{E}\left\{\int_{0}^{T}\left(\frac{1}{y C^{0}(s)}\right)^{\alpha} 2^{\alpha-1}\left[\kappa_{1}^{\alpha}+\kappa_{2}^{\alpha}\left(y C^{0}(s)\right)^{\alpha}\right] d s\right\} \\
& \leq 2^{\alpha-1}\left[\frac{\kappa_{1}^{\alpha}}{y^{\alpha}} \tilde{\mathbb{E}}\left\{\int_{0}^{T}\left(\frac{1}{C^{0}(s)}\right)^{\alpha} d s\right\}+T \kappa_{2}^{\alpha}\right] \\
& \leq \kappa\left(1+\frac{1}{y^{\alpha}}\right),
\end{aligned}
$$

where $\kappa>0$ is a suitable constant independent of $t$ and $y$ (cf. (A-1)). 
From now on and throughout this Appendix, we will define $Y^{y}(s):=y C^{0}(s)$ (cf. (2.2)) under the measure $\tilde{\mathbb{P}}$; also we denote by $\mathcal{L}$ the infinitesimal generator associated to $Y$, i.e.

$$
(\mathcal{L} f)(y):=\frac{1}{2} \sigma_{C}^{2} y^{2} f^{\prime \prime}(y)+\hat{\mu}_{C} y f^{\prime}(y), \quad f \in C_{b}^{2}(\mathbb{R}),
$$

where $\hat{\mu}_{C}:=-\mu_{C}+\frac{1}{2} \sigma_{C}^{2}$ and $C_{b}^{2}(\mathbb{R})$ is the space of functions which are twice-continuously differentiable on $(0, \infty)$ and bounded with their first two derivatives.

Inspired by Stroock and Varadhan [62] we adopt the following

Definition A.2. Take measurable functions $h:[0, T] \times(0, \infty) \mapsto \mathbb{R}$ and $u:[0, T] \times(0, \infty) \mapsto \mathbb{R}$ such that

$$
\begin{gathered}
\tilde{\mathbb{E}}\left\{\int_{0}^{s} e^{-\bar{\mu} r}\left|h\left(t+r, Y^{y}(r)\right)\right| d r\right\}<\infty, \quad s \geq 0, \\
\tilde{\mathbb{E}}\left\{e^{-\bar{\mu} s}\left|u\left(t+s, Y^{y}(s)\right)\right|\right\}<\infty, \quad s \geq 0,
\end{gathered}
$$

for any $(t, y) \in[0, T] \times(0, \infty)$ arbitrary but fixed. We say that $u$ solves

$$
\left(\partial_{t}+\mathcal{L}-\bar{\mu}\right) u(t, y)=h(t, y), \quad(t, y) \in[0, T] \times(0, \infty)
$$

in the martingale sense if and only if the process

$$
M:=\left\{e^{-\bar{\mu} s} u\left(t+s, Y^{y}(s)\right)-\int_{0}^{s} e^{-\bar{\mu} r} h\left(t+r, Y^{y}(r)\right) d r, s \geq 0\right\}
$$

is a $\tilde{\mathbb{P}}$-martingale.

Remark A.3. For any adapted, bounded process $Z:=\{Z(s), s \geq 0\}$, if $u$ and $h$ are as in Definition A.2 and $M$ of $(\mathrm{A}-3)$ is a $\tilde{\mathbb{P}}$-martingale, then the process

$$
\begin{aligned}
N:=\left\{e^{-\bar{\mu} s-\int_{0}^{s} Z(t+r) d r}\right. & u\left(t+s, Y^{y}(s)\right) \\
& \left.-\int_{0}^{s} e^{-\bar{\mu} r-\int_{0}^{r} Z(t+v) d v}\left[h\left(t+r, Y^{y}(r)\right)+Z(t+r) u\left(t+r, Y^{y}(r)\right)\right] d r, s \geq 0\right\}
\end{aligned}
$$

is a $\tilde{\mathbb{P}}$-martingale as well (cf. for instance [46], Remark 1.3).

Denote by $C_{b}^{\infty}$ the space of functions which are differentiable infinitely many times and which are bounded with all their derivatives. In order to set our problem in a suitable space we define a real valued function $w$ by

$$
w(y):=\frac{y}{1+y} \quad y \geq 0 .
$$

This is a positive, increasing, $C_{b}^{\infty}$-function on $[0,+\infty)$ and it is not hard to see that

$$
\tilde{\mathbb{E}}\left\{\int_{0}^{T} e^{-\rho s} \frac{1}{w\left(Y^{y}(s)\right)} d s\right\}<\frac{1}{\rho}+\frac{1}{y}\left[\frac{1}{\rho+\mu_{F}+\frac{1}{2} \sigma_{C}^{2}-\bar{\mu}}\right]
$$

for any $\rho>0$ and $\rho \neq \bar{\mu}-\mu_{F}-\frac{1}{2} \sigma_{C}^{2}$, by $(\mathrm{A}-1)$.

Definition A.4. For $w$ as in (A-4) we write

$$
\|f\|_{w, \infty}:=\sup _{(t, y) \in[0, T] \times[0, \infty)}|w(y) f(t, y)|
$$

and define

$$
\mathcal{C}_{b}^{w}([0, T] \times[0, \infty)):=\left\{f: f \in C([0, T] \times(0, \infty)) \text { and }\|f\|_{w, \infty}<\infty\right\}
$$


It easily follows that $\|\cdot\|_{w, \infty}$ is a norm and that $\mathcal{C}_{b}^{w}([0, T] \times[0, \infty))$ is a Banach space. Now we study a penalized problem.

Proposition A.5. For any given $\epsilon>0$ there exists a unique $u^{\epsilon} \in \mathcal{C}_{b}^{w}([0, T] \times[0, \infty))$ that solves

$$
\left\{\begin{array}{l}
\left(\partial_{t}+\mathcal{L}-\bar{\mu}\right) u^{\epsilon}(t, y)=-R_{c}(y)-\frac{1}{\epsilon}\left(\frac{c_{-}}{f_{C}}-u^{\epsilon}(t, y)\right)^{+}+\frac{1}{\epsilon}\left(u^{\epsilon}(t, y)-\frac{c_{+}}{f_{C}}\right)^{+} \\
u^{\epsilon}(T, y)=\frac{c_{-}}{f_{C}}
\end{array}\right.
$$

in the martingale sense of Definition A.2.

Proof. Fix $\epsilon>0$ and note that

$$
\begin{gathered}
-\frac{1}{\epsilon}\left(\frac{c_{-}}{f_{C}}-u^{\epsilon}\right)^{+}=\frac{1}{\epsilon} u^{\epsilon}-\frac{1}{\epsilon}\left(\frac{c_{-}}{f_{C}} \vee u^{\epsilon}\right), \\
\frac{1}{\epsilon}\left(u^{\epsilon}-\frac{c_{+}}{f_{C}}\right)^{+}=\frac{1}{\epsilon} u^{\epsilon}-\frac{1}{\epsilon}\left(\frac{c_{+}}{f_{C}} \wedge u^{\epsilon}\right) .
\end{gathered}
$$

From Remark A.3, with $u:=u^{\epsilon}, h:=-R_{c}-\frac{1}{\epsilon}\left(\frac{c_{-}}{f_{C}}-u^{\epsilon}\right)^{+}+\frac{1}{\epsilon}\left(u^{\epsilon}-\frac{c_{+}}{f_{C}}\right)^{+}$and $Z=\frac{1}{\epsilon}$, it follows that (A-8) may be rewritten as

$$
\left\{\begin{array}{l}
\left(\partial_{t}+\mathcal{L}-\left(\bar{\mu}+\frac{2}{\epsilon}\right)\right) u^{\epsilon}(t, y)=-R_{c}(y)-\frac{1}{\epsilon}\left(\frac{c_{-}}{f_{C}} \vee u^{\epsilon}(t, y)\right)-\frac{1}{\epsilon}\left(u^{\epsilon}(t, y) \wedge \frac{c_{+}}{f_{C}}\right) \\
u^{\epsilon}(T, y)=\frac{c_{-}}{f_{C}}
\end{array}\right.
$$

and the solution of (A-9) in the martingale sense (cf. Definition A.2), if it exists, is given by

$$
\begin{gathered}
u^{\epsilon}(t, y)=\tilde{\mathbb{E}}\left\{\frac{c_{-}}{f_{C}} e^{-\bar{\mu}(T-t)}+\int_{0}^{T-t} e^{-\left(\bar{\mu}+\frac{2}{\epsilon}\right) s}\left[R_{c}\left(Y^{y}(s)\right)+\frac{1}{\epsilon}\left(\frac{c_{-}}{f_{C}} \vee u^{\epsilon}\left(t+s, Y^{y}(s)\right)\right)\right.\right. \\
\left.+\frac{1}{\epsilon}\left(\frac{c_{+}}{f_{C}} \wedge u^{\epsilon}\left(t+s, Y^{y}(s)\right)\right) d s\right\} .
\end{gathered}
$$

We now show that $(\mathrm{A}-10)$ admits a unique solution in $\mathcal{C}_{b}^{w}([0, T] \times[0, \infty))$ by a fixed point argument. For $g \in \mathcal{C}_{b}^{w}([0, T] \times[0, \infty))$ we define the operator $\mathcal{T}^{\epsilon}$ by

$$
\begin{gathered}
\left(\mathcal{T}^{\epsilon} g\right)(t, y)=\tilde{\mathbb{E}}\left\{\frac{c_{-}}{f_{C}} e^{-\bar{\mu}(T-t)}+\int_{0}^{T-t} e^{-\left(\bar{\mu}+\frac{2}{\epsilon}\right) s}\left[R_{c}\left(Y^{y}(s)\right)+\frac{1}{\epsilon}\left(\frac{c_{-}}{f_{C}} \vee g\left(t+s, Y^{y}(s)\right)\right)\right.\right. \\
\left.\left.+\frac{1}{\epsilon}\left(\frac{c_{+}}{f_{C}} \wedge g\left(t+s, Y^{y}(s)\right)\right)\right] d s\right\}
\end{gathered}
$$

that maps $\mathcal{C}_{b}^{w}([0, T] \times[0, \infty))$ into itself. In order to prove that $(t, y) \mapsto \mathcal{T}^{\epsilon} g(t, y)$ is indeed continuous, take $\left(t_{1}, y_{1}\right)$ and $\left(t_{2}, y_{2}\right)$ in $[0, T] \times(0, \infty)$ (without loss of generality we may take, $t_{2}>t_{1}$ and $y_{2}>y_{1}>\delta$ for some $\left.\delta>0\right)$ and notice that

$$
\begin{aligned}
\left|\left(\mathcal{T}^{\epsilon} g\right)\left(t_{1}, y_{1}\right)-\left(\mathcal{T}^{\epsilon} g\right)\left(t_{2}, y_{2}\right)\right| & \leq\left|\left(\mathcal{T}^{\epsilon} g\right)\left(t_{1}, y_{1}\right)-\left(\mathcal{T}^{\epsilon} g\right)\left(t_{2}, y_{1}\right)\right|+\left|\left(\mathcal{T}^{\epsilon} g\right)\left(t_{2}, y_{1}\right)-\left(\mathcal{T}^{\epsilon} g\right)\left(t_{2}, y_{2}\right)\right| \\
& =:(I)+(I I) .
\end{aligned}
$$


Then, for $(I)$ we have

$$
\begin{aligned}
&(I) \leq\left|\frac{c_{-}}{f_{C}}\left(e^{-\bar{\mu}\left(T-t_{1}\right)}-e^{-\bar{\mu}\left(T-t_{2}\right)}\right)\right| \\
&+\left|\tilde{\mathbb{E}}\left\{\int_{0}^{T-t_{1}} e^{-\left(\bar{\mu}+\frac{2}{\epsilon}\right) s} R_{c}\left(Y^{y_{1}}(s)\right) d s-\int_{0}^{T-t_{2}} e^{-\left(\bar{\mu}+\frac{2}{\epsilon}\right) s} R_{c}\left(Y^{y_{1}}(s)\right) d s\right\}\right| \\
&+\mid \tilde{\mathbb{E}}\left\{\int_{0}^{T-t_{1}} e^{-\left(\bar{\mu}+\frac{2}{\epsilon}\right) s} \frac{1}{\epsilon}\left(\frac{c_{-}}{f_{C}} \vee g\left(t_{1}+s, Y^{y_{1}}(s)\right)\right) d s\right. \\
&\left.\quad-\int_{0}^{T-t_{2}} e^{-\left(\bar{\mu}+\frac{2}{\epsilon}\right) s} \frac{1}{\epsilon}\left(\frac{c_{-}}{f_{C}} \vee g\left(t_{2}+s, Y^{y_{1}}(s)\right)\right) d s\right\} \mid \\
& \quad-\mid \tilde{\mathbb{E}}\left\{\int_{0}^{T-t_{1}} e^{-\left(\bar{\mu}+\frac{2}{\epsilon}\right) s} \frac{1}{\epsilon}\left(\frac{c_{+}}{f_{C}} \wedge g\left(t_{1}+s, Y^{y_{1}}(s)\right)\right) d s\right. \\
&\left.-\int_{0}^{T-t_{2}} e^{-\left(\bar{\mu}+\frac{2}{\epsilon}\right) s} \frac{1}{\epsilon}\left(\frac{c_{+}}{f_{C}} \wedge g\left(t_{2}+s, Y^{y_{1}}(s)\right)\right) d s\right\} \mid .
\end{aligned}
$$

The second term on the right-hand side of (A-12) reads

$$
\begin{aligned}
& \left|\tilde{\mathbb{E}}\left\{\int_{0}^{T-t_{1}} e^{-\left(\bar{\mu}+\frac{2}{\epsilon}\right) s} R_{c}\left(Y^{y_{1}}(s)\right) d s-\int_{0}^{T-t_{2}} e^{-\left(\bar{\mu}+\frac{2}{\epsilon}\right) s} R_{c}\left(Y^{y_{1}}(s)\right) d s\right\}\right| \\
& \quad=\tilde{\mathbb{E}}\left\{\int_{T-t_{2}}^{T-t_{1}} e^{-\left(\bar{\mu}+\frac{2}{\epsilon}\right) s} R_{c}\left(Y^{y_{1}}(s)\right) d s\right\} .
\end{aligned}
$$

From Lemma A.1 and dominated convergence we obtain that the right-hand side of (A-13) converges to zero as soon as $t_{1} \rightarrow t_{2}$.

On the other hand, we shall use dominated convergence to show that the third and the fourth terms on the right-hand side of (A-12) converge to zero as $t_{1} \rightarrow t_{2}$. We will provide full details only for the third term as the same arguments apply to the fourth one. Observe that

$$
\begin{aligned}
& \left|\int_{0}^{T-t_{1}} e^{-\left(\bar{\mu}+\frac{2}{\epsilon}\right) s} \frac{1}{\epsilon}\left(\frac{c_{-}}{f_{C}} \vee g\left(t_{1}+s, Y^{y_{1}}(s)\right)\right) d s-\int_{0}^{T-t_{2}} e^{-\left(\bar{\mu}+\frac{2}{\epsilon}\right) s} \frac{1}{\epsilon}\left(\frac{c_{-}}{f_{C}} \vee g\left(t_{2}+s, Y^{y_{1}}(s)\right)\right) d s\right| \\
& \quad \leq \int_{0}^{T} e^{-\left(\bar{\mu}+\frac{2}{\epsilon}\right) s} \frac{w\left(Y^{y_{1}}(s)\right)}{w\left(Y^{y_{1}}(s)\right)}\left[\left|\frac{c_{-}}{f_{C}} \vee g\left(t_{1}+s, Y^{y_{1}}(s)\right)\right|+\left|\frac{c_{-}}{f_{C}} \vee g\left(t_{2}+s, Y^{y_{1}}(s)\right)\right|\right] d s \\
& \quad \leq 2\left\|\frac{c_{-}}{f_{C}} \vee g\right\|_{w, \infty} \int_{0}^{T} e^{-\left(\bar{\mu}+\frac{2}{\epsilon}\right) s} \frac{1}{w\left(Y^{y_{1}}(s)\right)} d s
\end{aligned}
$$

Using (A-5) with $\rho=\bar{\mu}+2 / \epsilon$ and recalling that $y_{2}>y_{1}>\delta$, one may easily verify that the last expression in (A-14) is independent of $t_{1}, t_{2}, y_{1}, y_{2}$ and it is $\tilde{\mathbb{P}}$-integrable. Therefore, from (A-14) and dominated convergence

$$
\lim _{t_{1} \rightarrow t_{2}}\left|\left(\mathcal{T}^{\epsilon} g\right)\left(t_{1}, y_{1}\right)-\left(\mathcal{T}^{\epsilon} g\right)\left(t_{2}, y_{1}\right)\right|=0
$$

Analogously (II) has three terms and it reads

$$
\begin{aligned}
(I I) \leq & \mid \tilde{\mathbb{E}}\left\{\int_{0}^{T-t_{2}} e^{-\left(\bar{\mu}+\frac{2}{\epsilon}\right) s}\left[R_{c}\left(Y^{y_{1}}(s)\right)-R_{c}\left(Y^{y_{2}}(s)\right)\right] d s\right\} \\
& +\frac{1}{\epsilon} \tilde{\mathbb{E}}\left\{\int_{0}^{T-t_{2}} e^{-\left(\bar{\mu}+\frac{2}{\epsilon}\right) s}\left[\left(\frac{c_{-}}{f_{C}} \vee g\left(t_{2}+s, Y^{y_{1}}(s)\right)\right)-\left(\frac{c_{-}}{f_{C}} \vee g\left(t_{2}+s, Y^{y_{2}}(s)\right)\right)\right] d s\right\} \\
& +\frac{1}{\epsilon} \tilde{\mathbb{E}}\left\{\int_{0}^{T-t_{2}} e^{-\left(\bar{\mu}+\frac{2}{\epsilon}\right) s}\left[\left(\frac{c_{+}}{f_{C}} \wedge g\left(t_{2}+s, Y^{y_{1}}(s)\right)\right)-\left(\frac{c_{+}}{f_{C}} \wedge g\left(t_{2}+s, Y^{y_{2}}(s)\right)\right)\right] d s\right\} \mid .
\end{aligned}
$$


Concavity of $R$ implies that

$$
\lim _{y_{1} \rightarrow y_{2}} \tilde{\mathbb{E}}\left\{\int_{0}^{T-t_{2}} e^{-\left(\bar{\mu}+\frac{2}{\epsilon}\right) s}\left|R_{c}\left(Y^{y_{1}}(s)\right)-R_{c}\left(Y^{y_{2}}(s)\right)\right| d s\right\}=0,
$$

by monotone convergence theorem.

It is not too hard to see that we can use estimates as the ones that led to (A-14) and dominated convergence to show that the second and the third terms in (A-15) go to zero as $y_{1} \rightarrow y_{2}$. Since the lower bound $\delta$ on $y_{1}$ and $y_{2}$ is arbitrary, we conclude that $(t, y) \mapsto\left(\mathcal{T}^{\epsilon} g\right)(t, y) \in C([0, T] \times(0, \infty))$ for all $g \in \mathcal{C}_{b}^{w}([0, T] \times[0, \infty))$.

Our next step is proving that $\left\|\mathcal{T}^{\epsilon} g\right\|_{w, \infty}<\infty$. Consider again $g \in \mathcal{C}_{b}^{w}([0, T] \times[0, \infty))$ and notice that

$$
\begin{aligned}
\left|w(y)\left(\mathcal{T}^{\epsilon} g\right)(t, y)\right| \leq & w(y) \tilde{\mathbb{E}}\left\{\int_{0}^{T-t} e^{-\left(\bar{\mu}+\frac{2}{\epsilon}\right) s} R_{c}\left(Y^{y}(s)\right) d s\right\}+w(y) \frac{c_{-}}{f_{C}} \\
& +\frac{1}{\epsilon} w(y) \tilde{\mathbb{E}}\left\{\int_{0}^{T-t} e^{-\left(\bar{\mu}+\frac{2}{\epsilon}\right) s} \frac{w\left(Y^{y}(s)\right)}{w\left(Y^{y}(s)\right)}\left[\left(\frac{c_{-}}{f_{C}} \vee g\right)+\left(\frac{c_{+}}{f_{C}} \wedge g\right)\right]\left(t+s, Y^{y}(s)\right) d s\right\} \\
\leq & \kappa w(y)\left(1+\frac{1}{y}\right)+w(y) \frac{c_{-}}{f_{C}} \\
& +\frac{w(y)}{\epsilon} \tilde{\mathbb{E}}\left\{\int_{0}^{T} e^{-\left(\bar{\mu}+\frac{2}{\epsilon}\right) s} \frac{d s}{w\left(Y^{y}(s)\right)}\right\}\left[\left\|\frac{c_{-}}{f_{C}} \vee g\right\|_{w, \infty}+\left\|\frac{c_{-}}{f_{C}} \wedge g\right\|_{w, \infty}\right],
\end{aligned}
$$

where we have used Lemma A.1 to find the first term in the last expression above and the same arguments as in (A-14) for the third one. Finally, recalling (A-5) and taking the supremum for $(t, y) \in[0, T] \times[0, \infty)$ we conclude that $\left\|\mathcal{T}^{\epsilon} g\right\|_{w, \infty}<\infty$.

To complete the proof we have now to show that $\mathcal{T}^{\epsilon}$ is a contraction. Take $g_{1}, g_{2} \in \mathcal{C}_{b}^{w}([0, T] \times$ $[0, \infty))$. Then, arguments as those employed to obtain (A-16) and (A-5) with $\rho=\bar{\mu}+2 / \epsilon$ give

$$
\begin{aligned}
\mid w(y) & \left(\mathcal{T}^{\epsilon} g_{1}-\mathcal{T}^{\epsilon} g_{2}\right)(t, y) \mid \\
\leq & w(y)\left|\tilde{\mathbb{E}}\left\{\int_{0}^{T-t} e^{-\left(\bar{\mu}+\frac{2}{\epsilon}\right) s}\left[\frac{1}{\epsilon}\left(\frac{c_{-}}{f_{C}} \vee g_{1}\right)-\frac{1}{\epsilon}\left(\frac{c_{-}}{f_{C}} \vee g_{2}\right)\right]\left(t+s, Y^{y}(s)\right) d s\right\}\right| \\
& +w(y)\left|\tilde{\mathbb{E}}\left\{\int_{0}^{T-t} e^{-\left(\bar{\mu}+\frac{2}{\epsilon}\right) s}\left[\frac{1}{\epsilon}\left(\frac{c_{+}}{f_{C}} \wedge g_{1}\right)-\frac{1}{\epsilon}\left(\frac{c_{+}}{f_{C}} \wedge g_{2}\right)\right]\left(t+s, Y^{y}(s)\right) d s\right\}\right| \\
\leq & 2 \frac{w(y)}{\epsilon}\left\|g_{1}-g_{2}\right\|_{w, \infty}\left[\frac{1}{y}\left(\frac{\epsilon}{2+\left(\mu_{F}+\frac{1}{2} \sigma_{C}^{2}\right) \epsilon}\right)+\frac{\epsilon}{2+\bar{\mu} \epsilon}\right] \\
= & w(y)\left\|g_{1}-g_{2}\right\|_{w, \infty}\left[\frac{1}{y}\left(\frac{1}{1+\left(\mu_{F}+\frac{1}{2} \sigma_{C}^{2}\right) \frac{\epsilon}{2}}\right)+\frac{1}{1+\frac{\bar{\mu} \epsilon}{2}}\right] .
\end{aligned}
$$

Set $c_{1}:=1 /\left(1+\left(\mu_{F}+\frac{1}{2} \sigma_{C}^{2}\right) \frac{\epsilon}{2}\right)$ and $c_{2}:=1 /\left(1+\frac{\bar{\mu} \epsilon}{2}\right)$. Then,

$$
w(y)\left[\frac{c_{1}}{y}+c_{2}\right] \leq c_{1} \vee c_{2}<1
$$

and $\mathcal{T}^{\epsilon}$ is a contraction. Hence, there exists a unique solution of the penalized problem (A-8) in $\mathcal{C}_{b}^{w}([0, T] \times[0, \infty))$, by Banach fixed point theorem.

From Definition A.2 and Proposition A.5 it follows

Corollary A.6. For any $(t, y) \in[0, T] \times(0, \infty)$ the process $H^{t, y}:=\left\{H^{t, y}(s), s \geq 0\right\}$ defined by

$$
\begin{aligned}
H^{t, y}(s):= & e^{-\bar{\mu} s} u^{\epsilon}\left(t+s, Y^{y}(s)\right) \\
& +\int_{0}^{s} e^{-\bar{\mu} r}\left[R_{c}\left(Y^{y}(r)\right)+\frac{1}{\epsilon}\left(\frac{c_{-}}{f_{C}}-u^{\epsilon}\left(t+r, Y^{y}(r)\right)\right)^{+}-\frac{1}{\epsilon}\left(u^{\epsilon}\left(t+r, Y^{y}(r)\right)-\frac{c_{+}}{f_{C}}\right)^{+}\right] d r
\end{aligned}
$$


is a continuous $\tilde{\mathbb{P}}$-martingale.

Proposition A.7. Define $\mathcal{A}:=\{\nu: \Omega \times[0, T] \mapsto[0,1]$, adapted $\}$. Then, the solution of the penalized problem (A-8) may be written as

$$
u^{\epsilon}(t, y)=\sup _{\nu_{1} \in \mathcal{A}} \inf _{\nu_{2} \in \mathcal{A}} \Xi^{\epsilon}\left(t, y ; \nu_{1}, \nu_{2}\right)=\inf _{\nu_{2} \in \mathcal{A}} \sup _{\nu_{1} \in \mathcal{A}} \Xi^{\epsilon}\left(t, y ; \nu_{1}, \nu_{2}\right),
$$

where

$$
\begin{gathered}
\Xi^{\epsilon}\left(t, y ; \nu_{1}, \nu_{2}\right):=\tilde{\mathbb{E}}\left\{\int_{0}^{T-t} \quad e^{-\bar{\mu} r-\frac{1}{\epsilon} \int_{0}^{r}\left(\nu_{1}(\alpha)+\nu_{2}(\alpha)\right) d \alpha}\left[R_{c}\left(Y^{y}(r)\right)+\frac{1}{\epsilon} \nu_{1}(r) \frac{c_{-}}{f_{C}}+\frac{1}{\epsilon} \nu_{2}(r) \frac{c_{+}}{f_{C}}\right] d r\right. \\
\left.+\frac{c_{-}}{f_{C}} e^{-\bar{\mu}(T-t)-\frac{1}{\epsilon} \int_{0}^{T-t}\left(\nu_{1}(\alpha)+\nu_{2}(\alpha)\right) d \alpha}\right\} .
\end{gathered}
$$

Proof. For any $\nu_{1}, \nu_{2} \in \mathcal{A}$ and $s \leq T-t$, we may write

$$
\begin{aligned}
u^{\epsilon}(t, y) & =\tilde{\mathbb{E}}\left\{e^{-\bar{\mu} s-\frac{1}{\epsilon} \int_{0}^{s}\left(\nu_{1}(\alpha)+\nu_{2}(\alpha)\right) d \alpha} u^{\epsilon}\left(t+s, Y^{y}(s)\right)\right. \\
& +\int_{0}^{s} e^{-\bar{\mu} r-\frac{1}{\epsilon} \int_{0}^{r}\left(\nu_{1}(\alpha)+\nu_{2}(\alpha)\right) d \alpha}\left[R_{c}\left(Y^{y}(r)\right)+\frac{1}{\epsilon}\left(\frac{c_{-}}{f_{C}}-u^{\epsilon}\left(t+r, Y^{y}(r)\right)\right)^{+}\right. \\
& \left.\left.-\frac{1}{\epsilon}\left(u^{\epsilon}\left(t+r, Y^{y}(r)\right)-\frac{c_{+}}{f_{C}}\right)^{+}+\frac{1}{\epsilon}\left(\nu_{1}(r)+\nu_{2}(r)\right) u^{\epsilon}\left(t+r, Y^{y}(r)\right)\right] d r\right\},
\end{aligned}
$$

by Corollary A.6 and Remark A.3, with $u:=u^{\epsilon}, h:=-R_{c}-\frac{1}{\epsilon}\left(\frac{c_{-}}{f_{C}}-u^{\epsilon}\right)^{+}+\frac{1}{\epsilon}\left(u^{\epsilon}-\frac{c_{+}}{f_{C}}\right)^{+}$and $Z(s):=\frac{1}{\epsilon}\left[\nu_{1}(s)+\nu_{2}(s)\right]$. Notice now that

$$
-\frac{1}{\epsilon}\left(u^{\epsilon}-\frac{c_{+}}{f_{C}}\right)^{+}+\frac{1}{\epsilon} \nu_{2} u^{\epsilon}= \begin{cases}-\frac{1}{\epsilon}\left(u^{\epsilon}-\frac{c_{+}}{f_{C}}\right)+\frac{1}{\epsilon} \nu_{2} u^{\epsilon} & \text { on }\left\{u^{\epsilon} \geq \frac{c_{+}}{f_{C}}\right\} \\ \frac{1}{\epsilon} \nu_{2} u^{\epsilon} & \text { on }\left\{u^{\epsilon}<\frac{c_{+}}{f_{C}}\right\}\end{cases}
$$

implies $-\frac{1}{\epsilon}\left(u^{\epsilon}-\frac{c_{+}}{f_{C}}\right)^{+}+\frac{1}{\epsilon} \nu_{2} u^{\epsilon} \leq \frac{1}{\epsilon} \nu_{2} \frac{c_{+}}{f_{C}}$ with equality if and only if we take

$$
\nu_{2}^{*}:= \begin{cases}1 & \text { on }\left\{u^{\epsilon} \geq \frac{c_{+}}{f_{C}}\right\} \\ 0 & \text { on }\left\{u^{\epsilon}<\frac{c_{+}}{f_{C}}\right\}\end{cases}
$$

Similarly,

$$
\frac{1}{\epsilon}\left(\frac{c_{-}}{f_{C}}-u^{\epsilon}\right)^{+}+\frac{1}{\epsilon} \nu_{1} u^{\epsilon}= \begin{cases}\frac{1}{\epsilon}\left(\frac{c_{-}}{f_{C}}-u^{\epsilon}\right)+\frac{1}{\epsilon} \nu_{1} u^{\epsilon} & \text { on }\left\{u^{\epsilon} \leq \frac{c_{-}}{f_{C}}\right\} \\ \frac{1}{\epsilon} \nu_{1} u^{\epsilon} & \text { on }\left\{u^{\epsilon}>\frac{c_{-}}{f_{C}}\right\}\end{cases}
$$

implies $\frac{1}{\epsilon}\left(\frac{c_{-}}{f_{C}}-u^{\epsilon}\right)^{+}+\frac{1}{\epsilon} \nu_{1} u^{\epsilon} \geq \frac{1}{\epsilon} \nu_{1} \frac{c_{-}}{f_{C}}$ with equality if and only if we take

$$
\nu_{1}^{*}:= \begin{cases}1 & \text { on }\left\{u^{\epsilon} \leq \frac{c_{-}}{f_{C}}\right\} \\ 0 & \text { on }\left\{u^{\epsilon}>\frac{c_{-}}{f_{C}}\right\}\end{cases}
$$

In particular, (A-21) evaluated at $s=T-t$, together with (A-22) and (A-25) give

$$
u^{\epsilon}(t, y) \leq \Xi^{\epsilon}\left(t, y ; \nu_{1}^{*}, \nu_{2}\right) \quad \text { for all } \nu_{2} \in \mathcal{A}
$$

and

$$
u^{\epsilon}(t, y) \geq \Xi^{\epsilon}\left(t, y ; \nu_{1}, \nu_{2}^{*}\right) \quad \text { for all } \nu_{2} \in \mathcal{A}
$$

Then (A-19) follows. 
Since $\Xi^{\epsilon}\left(t, y ; \nu_{1}, \nu_{2}\right) \geq 0$ for all $\nu_{1}, \nu_{2} \in \mathcal{A}$ (cf. (A-20)), then

$$
u^{\epsilon}(t, y) \geq 0 \quad \text { for all }(t, y) \in[0, T] \times(0, \infty)
$$

by (A-19).

Proposition A.8. One has

$$
\lim _{\epsilon \downarrow 0}\left\|\left(u^{\epsilon}-\frac{c_{+}}{f_{C}}\right)^{+}\right\|_{w, \infty}=0
$$

and

$$
\lim _{\epsilon \downarrow 0}\left\|\left(\frac{c_{-}}{f_{C}}-u^{\epsilon}\right)^{+}\right\|_{w, \infty}=0 .
$$

Proof. For any $\nu_{1}, \nu_{2} \in \mathcal{A}$ we may write

$$
\begin{aligned}
\frac{c_{+}}{f_{C}} & =\frac{c_{+}}{f_{C}} e^{-\bar{\mu}(T-t)-\frac{1}{\epsilon} \int_{0}^{T-t}\left(\nu_{1}(\alpha)+\nu_{2}(\alpha)\right) d \alpha} \\
& +\int_{0}^{T-t} e^{-\bar{\mu} r-\frac{1}{\epsilon} \int_{0}^{r}\left(\nu_{1}(\alpha)+\nu_{2}(\alpha)\right) d \alpha} \frac{c_{+}}{f_{C}}\left(\bar{\mu}+\frac{1}{\epsilon}\left[\nu_{1}(r)+\nu_{2}(r)\right]\right) d r
\end{aligned}
$$

by an integration by parts. Then from (A-19) and (A-20) it follows

$$
\begin{aligned}
u^{\epsilon}(t, y)-\frac{c_{+}}{f_{C}} & \leq \inf _{\nu_{2} \in \mathcal{A}} \sup _{\nu_{1} \in \mathcal{A}} \tilde{\mathbb{E}}\left\{\int_{0}^{T-t} e^{-\bar{\mu} r-\frac{1}{\epsilon} \int_{0}^{r}\left(\nu_{1}(\alpha)+\nu_{2}(\alpha)\right) d \alpha}\left[R_{c}\left(Y^{y}(r)\right)-\bar{\mu} \frac{c_{+}}{f_{C}}\right] d r\right\} \\
& \leq \tilde{\mathbb{E}}\left\{\int_{0}^{T-t} e^{-\bar{\mu} r-\frac{1}{\epsilon} r} R_{c}\left(Y^{y}(r)\right) d r\right\} \\
& \leq \tilde{\mathbb{E}}\left\{\int_{0}^{T-t} e^{-2\left(\bar{\mu}+\frac{1}{\epsilon}\right) r} d r\right\}^{\frac{1}{2}} \tilde{\mathbb{E}}\left\{\int_{0}^{T-t} R_{c}^{2}\left(Y^{y}(r)\right) d r\right\}^{\frac{1}{2}} \\
& \leq\left[\frac{\epsilon}{2(1+\bar{\mu} \epsilon)}\right]^{\frac{1}{2}}\left[\kappa\left(1+\frac{1}{y^{2}}\right)\right]^{\frac{1}{2}},
\end{aligned}
$$

where the third expression follows by Hölder inequality and Lemma A.1 implies the last one.

Similarly,

$$
\begin{aligned}
u^{\epsilon}(t, y)-\frac{c_{-}}{f_{C}} & \geq \inf _{\nu_{2} \in \mathcal{A}} \sup _{\nu_{1} \in \mathcal{A}} \tilde{\mathbb{E}}\left\{\int_{0}^{T-t} e^{-\bar{\mu} r-\frac{1}{\epsilon} \int_{0}^{r}\left(\nu_{1}(\alpha)+\nu_{2}(\alpha)\right) d \alpha}\left[R_{c}\left(Y^{y}(r)\right)-\bar{\mu} \frac{c_{-}}{f_{C}}\right] d r\right\} \\
& \geq-\tilde{\mathbb{E}}\left\{\int_{0}^{T-t} e^{-\bar{\mu} r-\frac{1}{\epsilon} r}\left[R_{c}\left(Y^{y}(r)\right)+\bar{\mu} \frac{c_{-}}{f_{C}}\right] d r\right\} \\
& \geq-\left[\frac{\epsilon}{2(1+\bar{\mu} \epsilon)}\right]^{\frac{1}{2}}\left[\kappa\left(1+\frac{1}{y^{2}}\right)\right]^{\frac{1}{2}}-\frac{\bar{\mu} c_{-}}{f_{C}}\left[\frac{\epsilon}{1+\bar{\mu} \epsilon}\right] .
\end{aligned}
$$

Hence, (A-28) and (A-27) follow from Definition A.4.

Before proving Theorem 3.4 we shall make further observations on $u^{\epsilon}$. Take $\sigma$ and $\tau$ arbitrary stopping times in $[0, T-t]$. From Corollary A.6, with $s$ replaced by $\sigma \wedge \tau$, we find

$$
\begin{aligned}
u^{\epsilon}(t, y)=\mathbb{E}\left\{e^{-\bar{\mu}(\tau \wedge \sigma)} u^{\epsilon}\left(t+\tau \wedge \sigma, Y^{y}(\tau \wedge \sigma)\right)\right. & \\
& +\int_{0}^{\tau \wedge \sigma} e^{-\bar{\mu} r}\left[R_{c}\left(Y^{y}(r)\right)+\frac{1}{\epsilon}\left(\frac{c_{-}}{f_{C}}-u^{\epsilon}\left(t+r, Y^{y}(r)\right)\right)^{+}\right. \\
& \left.\left.-\frac{1}{\epsilon}\left(u^{\epsilon}\left(t+r, Y^{y}(r)\right)-\frac{c_{+}}{f_{C}}\right)^{+}\right] d r\right\}
\end{aligned}
$$


Now, recalling that $u^{\epsilon}(T, y)=\frac{c_{-}}{f_{C}}$ and noting that $u^{\epsilon} \leq \frac{c_{+}}{f_{C}}+\left(u^{\epsilon}-\frac{c_{+}}{f_{C}}\right)^{+}$and $u^{\epsilon} \geq \frac{c_{-}}{f_{C}}-\left(\frac{c_{-}}{f_{C}}-u^{\epsilon}\right)^{+}$, we have

$$
\begin{aligned}
u^{\epsilon}(t, y) & \leq \mathbb{E}\left\{e^{-\bar{\mu} \tau} u^{\epsilon}\left(t+\tau, Y^{y}(\tau)\right) \mathbb{1}_{\{\tau<\sigma\}}+\frac{c_{+}}{f_{C}} e^{-\bar{\mu} \sigma} \mathbb{1}_{\{\sigma \leq \tau\}} \mathbb{1}_{\{\sigma<T-t\}}\right. \\
& +e^{-\bar{\mu} \sigma}\left(u^{\epsilon}\left(t+\sigma, Y^{y}(\sigma)\right)-\frac{c_{+}}{f_{C}}\right)^{+} \mathbb{1}_{\{\sigma \leq \tau\}} \mathbb{1}_{\{\sigma<T-t\}}+\frac{c_{-}}{f_{C}} e^{-\bar{\mu}(T-t)} \mathbb{1}_{\{\tau=\sigma=T-t\}} \\
& \left.+\int_{0}^{\tau \wedge \sigma} e^{-\bar{\mu} s}\left[R_{c}\left(Y^{y}(r)\right)+\frac{1}{\epsilon}\left(\frac{c_{-}}{f_{C}}-u^{\epsilon}\left(t+r, Y^{y}(r)\right)\right)^{+}\right] d r\right\}
\end{aligned}
$$

and

$$
\begin{aligned}
u^{\epsilon}(t, y) & \geq \mathbb{E}\left\{e^{-\bar{\mu} \sigma} u^{\epsilon}\left(t+\sigma, Y^{y}(\sigma)\right) \mathbb{1}_{\{\sigma \leq \tau\}} \mathbb{1}_{\{\sigma<T-t\}}+\frac{c_{-}}{f_{C}} e^{-\bar{\mu} \tau} \mathbb{1}_{\{\tau<\sigma\}}\right. \\
& -e^{-\bar{\mu} \tau}\left(\frac{c_{-}}{f_{C}}-u^{\epsilon}\left(t+\tau, Y^{y}(\tau)\right)\right)^{+} \mathbb{1}_{\{\tau<\sigma\}}+\frac{c_{-}}{f_{C}} e^{-\bar{\mu}(T-t)} \mathbb{1}_{\{\tau=\sigma=T-t\}} \\
& \left.+\int_{0}^{\tau \wedge \sigma} e^{-\bar{\mu} r}\left[R_{c}\left(Y^{y}(r)\right)-\frac{1}{\epsilon}\left(u^{\epsilon}\left(t+r, Y^{y}(r)\right)-\frac{c_{+}}{f_{C}}\right)^{+}\right] d r\right\} .
\end{aligned}
$$

We are now able to prove Theorem 3.4.

Proof of Theorem 3.4. From (3.8) and (A-33) we find

$$
\begin{aligned}
u^{\epsilon}(t, y)-v(t, y) \leq \inf _{\tau \in[0, T-t]} \sup _{\sigma \in[0, T-t]} \tilde{\mathbb{E}}\{ & e^{-\bar{\mu} \tau}\left(u^{\epsilon}\left(t+\tau, Y^{y}(\tau)\right)-\frac{c_{-}}{f_{C}}\right) \mathbb{I}_{\{\tau<\sigma\}} \\
& +e^{-\bar{\mu} \sigma}\left(u^{\epsilon}\left(t+\sigma, Y^{y}(\sigma)\right)-\frac{c_{+}}{f_{C}}\right)^{+} \mathbb{I}_{\{\sigma \leq \tau\}} \mathbb{I}_{\{\sigma<T-t\}} \\
& \left.+\frac{1}{\epsilon} \int_{0}^{\sigma \wedge \tau} e^{-\bar{\mu} r}\left(\frac{c_{-}}{f_{C}}-u^{\epsilon}\left(t+r, Y^{y}(r)\right)\right)^{+} d r\right\} .
\end{aligned}
$$

Take $\tau=\tau^{\epsilon}:=\inf \left\{s \in[0, T-t): u^{\epsilon}\left(t+s, Y^{y}(s)\right) \leq c_{-} / f_{C}\right\} \wedge(T-t)$ in (A-35) to obtain

$$
\begin{aligned}
u^{\epsilon}(t, y)-v(t, y) & \leq \sup _{\sigma \in[0, T-t]} \tilde{\mathbb{E}}\left\{e^{-\bar{\mu} \sigma}\left(u^{\epsilon}\left(t+\sigma, Y^{y}(\sigma)\right)-\frac{c_{+}}{f_{C}}\right)^{+}\right\} \\
& \leq \sup _{\sigma \in[0, T-t]} \tilde{\mathbb{E}}\left\{\frac{1}{w\left(Y^{y}(\sigma)\right)}\right\}\left\|\left(u^{\epsilon}-\frac{c_{+}}{f_{C}}\right)^{+}\right\| \|_{w, \infty} \\
& \leq\left[1+\frac{1}{y} \tilde{\mathbb{E}}\left\{\sup _{0 \leq s \leq T-t} \frac{1}{C^{0}(s)}\right\}\right]\left\|\left(u^{\epsilon}-\frac{c_{+}}{f_{C}}\right)^{+}\right\|_{w, \infty} .
\end{aligned}
$$

Arguing in a similar way and using (A-34) we also obtain

$$
u^{\epsilon}(t, y)-v(t, y) \geq-\left[1+\frac{1}{y} \tilde{\mathbb{E}}\left\{\sup _{0 \leq s \leq T-t} \frac{1}{C^{0}(s)}\right\}\right]\left\|\left(\frac{c_{-}}{f_{C}}-u^{\epsilon}\right)^{+}\right\|_{w, \infty} .
$$

Therefore (cf. Definition A.4)

$$
\left\|u^{\epsilon}-v\right\|_{w, \infty} \leq \kappa\left(\left\|\left(u^{\epsilon}-\frac{c_{+}}{f_{C}}\right)^{+}\right\|_{w, \infty}+\left\|\left(\frac{c_{-}}{f_{C}}-u^{\epsilon}\right)^{+}\right\|_{w, \infty}\right)
$$

for a suitable constant $\kappa>0$ depending only on $\hat{\sigma}_{C}, \hat{\mu}_{C}$ and $T>0$. Now, the right-hand side of (A-38) goes to zero as $\epsilon \rightarrow 0$ and $w v \in C([0, T] \times[0, \infty))$, thus implying $v \in C([0, T] \times(0, \infty))$.

Remark A.9. Note that for any $\delta>0$, one has $\left\|u^{\epsilon}-v\right\|_{w, \infty} \geq \delta /(1+\delta) \sup _{[0, T] \times[\delta, \infty)}\left|u^{\epsilon}-v\right|(t, y)$ and hence $u^{\epsilon} \rightarrow v$ uniformly on $[0, T] \times[\delta, \infty)$ as $\epsilon \rightarrow 0$. 


\section{A.2 Proof of Theorem 3.5}

For $\epsilon>0$ set

$$
\left\{\begin{array}{l}
\tau^{\epsilon}(t, y):=\inf \left\{s \in[0, T-t): u^{\epsilon}\left(t+s, Y^{y}(s)\right) \leq \frac{c_{-}}{f_{C}}\right\} \wedge(T-t), \\
\sigma^{\epsilon}(t, y):=\inf \left\{s \in[0, T-t): u^{\epsilon}\left(t+s, Y^{y}(s)\right) \geq \frac{c_{+}}{f_{C}}\right\} \wedge(T-t) .
\end{array}\right.
$$

Take $\delta>0$ arbitrary but fixed and define the first exit time of $Y$ from the half-plane $(\delta, \infty)$ by

$$
\tau_{\delta}(y):=\inf \left\{s \geq 0: Y^{y}(s) \leq \delta\right\} .
$$

Note that for all $y>0$, one finds

$$
\tau_{\delta}(y) \uparrow \infty \quad \text { as } \delta \downarrow 0, \tilde{\mathbb{P}} \text {-a.s. }
$$

as $\{0\}$ is a non-attainable boundary point for the process $Y$. For simplicity we set $\tau^{\epsilon} \equiv \tau^{\epsilon}(t, y)$, $\sigma^{\epsilon} \equiv \sigma^{\epsilon}(t, y)$ and $\tau_{\delta} \equiv \tau_{\delta}(y)$.

From Remark A.9 $u^{\epsilon} \rightarrow v$ uniformly on $[0, T] \times[\delta, \infty)$ as $\epsilon \downarrow 0$. Then, following the same arguments as in the proof of [19], Lemma 6.2 , we find that

$$
\begin{array}{ll}
\lim _{\epsilon \rightarrow 0} \tau^{*} \wedge \tau^{\epsilon} \wedge \tau_{\delta}=\tau^{*} \wedge \tau_{\delta} & \tilde{\mathbb{P}}_{\text {-a.s. }} \\
\lim _{\epsilon \rightarrow 0} \sigma^{*} \wedge \sigma^{\epsilon} \wedge \tau_{\delta}=\sigma^{*} \wedge \tau_{\delta} & \tilde{\mathbb{P}}_{\text {-a.s. }}
\end{array}
$$

for all $(t, y) \in[0, T] \times(0, \infty)$ and with $\tau^{*}$ and $\sigma^{*}$ as in (3.11). Therefore, we also have

$$
\lim _{\epsilon \rightarrow \infty} \sigma^{*} \wedge \sigma^{\epsilon} \wedge \tau^{*} \wedge \tau^{\epsilon} \wedge \tau_{\delta}=\sigma^{*} \wedge \tau^{*} \wedge \tau_{\delta} \quad \tilde{\mathbb{P}} \text {-a.s. }
$$

for all $(t, y) \in[0, T] \times(0, \infty)$.

Again, to simplify notation we set $\rho_{\delta, \epsilon}:=\sigma^{*} \wedge \sigma^{\epsilon} \wedge \tau^{*} \wedge \tau^{\epsilon} \wedge \tau_{\delta}$ and we obtain

$$
u^{\epsilon}(t, y)=\tilde{\mathbb{E}}\left\{e^{-\bar{\mu} \rho_{\delta, \epsilon}} u^{\epsilon}\left(t+\rho_{\delta, \epsilon}, Y^{y}\left(\rho_{\delta, \epsilon}\right)\right)+\int_{0}^{\rho_{\delta, \epsilon}} e^{-\bar{\mu} s} R_{c}\left(Y^{y}(s)\right) d s\right\}
$$

by (A-32). Taking limits as $\epsilon \rightarrow 0$ in (A-45), the left-hand side converges to $v$ by uniform convergence. On the other hand, we employ dominated convergence in the right-hand side. Thus we obtain

$$
v(t, y)=\tilde{\mathbb{E}}\left\{e^{-\bar{\mu} \sigma^{*} \wedge \tau^{*} \wedge \tau_{\delta}} v\left(t+\sigma^{*} \wedge \tau^{*} \wedge \tau_{\delta}, Y^{y}\left(\sigma^{*} \wedge \tau^{*} \wedge \tau_{\delta}\right)\right)+\int_{0}^{\sigma^{*} \wedge \tau^{*} \wedge \tau_{\delta}} e^{-\bar{\mu} s} R_{c}\left(Y^{y}(s)\right) d s\right\}
$$

by Remark A.9, (A-44) and continuity of $v$. Similarly, when $\delta \rightarrow 0$ in (A-46) one has

$$
v(t, y)=\tilde{\mathbb{E}}\left\{e^{-\bar{\mu} \sigma^{*} \wedge \tau^{*}} v\left(t+\sigma^{*} \wedge \tau^{*}, Y^{y}\left(\sigma^{*} \wedge \tau^{*}\right)\right)+\int_{0}^{\sigma^{*} \wedge \tau^{*}} e^{-\bar{\mu} s} R_{c}\left(Y^{y}(s)\right) d s\right\}
$$

by monotone convergence and (A-41) for the integral term, and by dominated convergence, (A-41) and continuity of $v$ for the other one.

Note that

$$
\begin{aligned}
& e^{-\bar{\mu} \sigma^{*} \wedge \tau^{*}} v\left(t+\sigma^{*} \wedge \tau^{*}, Y^{y}\left(\sigma^{*} \wedge \tau^{*}\right)\right) \\
& =e^{-\bar{\mu} \tau^{*}} \frac{c_{-}}{f_{C}} \mathbb{1}_{\left\{\tau^{*}<\sigma^{*}\right\}}+e^{-\bar{\mu} \sigma^{*}} \frac{c_{+}}{f_{C}} \mathbb{1}_{\left\{\sigma^{*} \leq \tau^{*}\right\}} \mathbb{1}_{\left\{\sigma^{*}<T-t\right\}}+e^{-\bar{\mu}(T-t)} \frac{c_{-}}{f_{C}} \mathbb{1}_{\left\{\sigma^{*}=\tau^{*}=T-t\right\}} \quad \tilde{\mathbb{P}}_{\text {-a.s. }}
\end{aligned}
$$


and therefore

$$
\begin{aligned}
v(t, y)=\tilde{\mathbb{E}}\{ & e^{-\bar{\mu} \tau^{*}} \frac{c_{-}}{f_{C}} \mathbb{1}_{\left\{\tau^{*}<\sigma^{*}\right\}}+e^{-\bar{\mu} \sigma^{*}} \frac{c_{+}}{f_{C}} \mathbb{1}_{\left\{\sigma^{*} \leq \tau^{*}\right\}} \mathbb{1}_{\left\{\sigma^{*}<T-t\right\}}+e^{-\bar{\mu}(T-t)} \frac{c_{-}}{f_{C}} \mathbb{1}_{\left\{\sigma^{*}=\tau^{*}=T-t\right\}} \\
& \left.+\int_{0}^{\tau^{*} \wedge \sigma^{*}} e^{-\bar{\mu} s} R_{c}\left(Y^{y}(r)\right) d r\right\} .
\end{aligned}
$$

It remains now to show that $\left(\tau^{*}, \sigma^{*}\right)$ is indeed a saddle point for the functional $\Psi$ of (3.9). Take an arbitrary stopping time $\sigma \in[0, T-t]$, define $\tau_{\delta, \epsilon}:=\tau^{*} \wedge \tau^{\epsilon} \wedge \tau_{\delta}$ and replace $\tau \wedge \sigma$ in (A-32) by $\sigma \wedge \tau_{\delta, \epsilon}$. It gives

$$
u^{\epsilon}(t, y) \leq \tilde{\mathbb{E}}\left\{e^{-\bar{\mu}\left(\sigma \wedge \tau_{\delta, \epsilon}\right)} u^{\epsilon}\left(t+\sigma \wedge \tau_{\delta, \epsilon}, Y^{y}\left(\sigma \wedge \tau_{\delta, \epsilon}\right)\right)+\int_{0}^{\sigma \wedge \tau_{\delta, \epsilon}} e^{-\bar{\mu} s} R_{c}\left(Y^{y}(s)\right) d s\right\} .
$$

First we let $\epsilon$ go to zero and then take limits as $\delta \downarrow 0$; using arguments as in (A-45)-(A-47) we obtain

$$
v(t, y) \leq \tilde{\mathbb{E}}\left\{e^{-\bar{\mu}\left(\sigma \wedge \tau^{*}\right)} v\left(t+\sigma \wedge \tau^{*}, Y^{y}\left(\sigma \wedge \tau^{*}\right)\right)+\int_{0}^{\sigma \wedge \tau^{*}} e^{-\bar{\mu} s} R_{c}\left(Y^{y}(s)\right) d s\right\} .
$$

From (3.11), (A-48) and the fact that $v \leq \frac{c_{+}}{f_{C}}$ we find

$$
\begin{aligned}
v(t, y) \leq \tilde{\mathbb{E}} & \left\{e^{-\bar{\mu} \tau^{*}} \frac{c_{-}}{f_{C}} \mathbb{1}_{\left\{\tau^{*}<\sigma\right\}}+e^{-\bar{\mu} \sigma} \frac{c_{+}}{f_{C}} \mathbb{1}_{\left\{\sigma \leq \tau^{*}\right\}} \mathbb{1}_{\{\sigma<T-t\}}+e^{-\bar{\mu}(T-t)} \frac{c_{-}}{f_{C}} \mathbb{1}_{\left\{\sigma=\tau^{*}=T-t\right\}}\right. \\
& \left.+\int_{0}^{\tau^{*} \wedge \sigma} e^{-\bar{\mu} s} R_{c}\left(Y^{y}(s)\right) d s\right\} .
\end{aligned}
$$

Analogously, take an arbitrary stopping time $\tau \in[0, T-t]$, define $\sigma_{\delta, \epsilon}:=\sigma^{*} \wedge \sigma^{\epsilon} \wedge \tau_{\delta}$ and set $\tau \wedge \sigma:=\tau \wedge \sigma_{\delta, \epsilon}$ in (A-32). Same arguments as in (A-50) and (A-51) give

$$
v(t, y) \geq \tilde{\mathbb{E}}\left\{e^{-\bar{\mu}\left(\sigma^{*} \wedge \tau\right)} v\left(t+\sigma^{*} \wedge \tau, Y^{y}\left(\sigma^{*} \wedge \tau\right)\right)+\int_{0}^{\sigma^{*} \wedge \tau} e^{-\bar{\mu} s} R_{c}\left(Y^{y}(s)\right) d s\right\},
$$

and hence

$$
\begin{aligned}
v(t, y) \geq \tilde{\mathbb{E}}\{ & e^{-\bar{\mu} \tau} \frac{c_{-}}{f_{C}} \mathbb{1}_{\left\{\tau<\sigma^{*}\right\}}+e^{-\bar{\mu} \sigma^{*}} \frac{c_{+}}{f_{C}} \mathbb{1}_{\left\{\sigma^{*} \leq \tau\right\}} \mathbb{1}_{\left\{\sigma^{*}<T-t\right\}}+e^{-\bar{\mu}(T-t)} \frac{c_{-}}{f_{C}} \mathbb{1}_{\left\{\sigma^{*}=\tau=T-t\right\}} \\
& \left.+\int_{0}^{\tau \wedge \sigma^{*}} e^{-\bar{\mu} s} R_{c}\left(Y^{y}(s)\right) d s\right\}
\end{aligned}
$$

by (3.11) and the bound $v \geq \frac{c_{-}}{f_{C}}$.

Acknowledgements: We wish to thank M.B. Chiarolla, J. Moriarty, G. Peskir, F. Riedel and G. Stabile for many useful discussions.

\section{References}

[1] A.B. Abel, J.C. Eberly, Optimal Investment with Costly Reversibility, Review of Economic Studies 63 (1996), pp. 581-593.

[2] M. Alario-Nazaret, J.P. Lepeltier, B. Marchal, Dynkin Games, Lecture Notes in Control and Information Sciences 43 (1982), pp. 23-32. 
[3] F.M. Baldursson, I. Karatzas, Irreversible Investment and Industry Equilibrium, Finance and Stochastics 1 (1997), pp. 69-89.

[4] P. Bank, N. El Karoui, A Stochastic Representation Theorem with Applications to Optimization and Obstacle Problems, The Annals of Probability 32 (2004), pp. 1030-1067.

[5] P. Bank, Optimal Control under a Dynamic Fuel Constraint, SIAM Journal on Control and Optimization 44 (2005), pp. 1529-1541.

[6] A. Bensoussan, A. Friedman, Nonlinear Variational Inequalities and Differential Games with Stopping Times, Journal of Functional Analysis 16 (1974), pp. 305-352.

[7] A. Bensoussan, A. Friedman, Non-zero Sum Stochastic Differential Games with Stopping Times and Free-Boundary Problems, Transactions of the American Mathematical Society 231 (1977), pp. 275-327.

[8] A. Bensoussan, J.L. Lions, Applications of Variational Inequalities to Stochastic Control, North Holland Publishing Company 1982.

[9] S. Bentolila, G. Bertola, Firing Costs and Labour Demand: How Bad is Eurosclerosis?, The Review of Economic Studies 57(3) (1990), pp. 381-402.

[10] P. Billingsley, Probability and Measures, John Wiley and Sons Inc., New York, 1986.

[11] J.M. Bismut, Sur un Problème de Dynkin, Z. Wahrsch. verw. Gebiete 39 (1977), pp. 31-53.

[12] F. Boetius, M. Kohlmann, Connections between Optimal Stopping and Singular Stochastic Control, Stochastic Processes and their Applications 77 (1998), pp. 253-281.

[13] F. Boetius, Bounded Variation Singular Stochastic Control and Dynkin Game, SIAM Journal on Control and Optimization 44 (2005), pp. 1289-1321.

[14] K. Burdzy, W. Kang, K. Ramanan, The Skorokhod Problem in a Time-Dependent Interval, Stochastic Processes and their Applications 119 (2009), pp. 428-454.

[15] M.B. Chiarolla, U.G. Haussmann, Optimal Control of Inflation: a Central Bank Problem, SIAM Journal on Control and Optimization 36 (1998), pp. 1099-1132.

[16] M.B. Chiarolla, U.G. Haussmann, Controlling Inflation: The Infinite Horizon Case, Applied Mathematics and Optimization 41 (2000), pp. 25-50.

[17] M.B. Chiarolla, U.G. Haussmann, On a Stochastic Irreversible Investment Problem, SIAM Journal on Control and Optimization 48 (2009), pp. 438-462.

[18] M.B. Chiarolla, G. Ferrari, Identifying the Free Boundary of a Stochastic, Irreversible Investment Problem via the Bank-El Karoui Representation Theorem, preprint (2011). arXiv:1108.4886. Submitted.

[19] M.B. Chiarolla, T. De Angelis, Analytical Pricing of American Bond Options in the HealthJarrow-Morton Model, preprint (2012). arXiv:1212.0781. Submitted.

[20] M.B. Chiarolla, G. Ferrari, F. Riedel, Generalized Kuhn-Tucker Conditions for Stochastic Irreversible Investments with Limited Resources, Center for Mathematical Economics Working paper no. 463 (2012). arXiv:1203.3757. Submitted.

[21] J. Cvitanic, I. Karatzas, Backward Stochastic Differential Equations with Reflection and Dynkin Games, Annals of Probability 24 (1996), pp. 2024-2056. 
[22] A.K. Dixit, R.S. Pindyck, Investment under Uncertainty, Princeton University Press, Princeton 1994.

[23] E.B. Dynkin, Game Variant of a Problem on Optimal Stopping, Soviet Mathematics Doklady 10 (1967), pp. 270-274.

[24] E. Ekström, G. Peskir, Optimal Stopping Games for Markov Processes, SIAM Journal on Control and Optimization 47(2) (2008), pp. 684-702.

[25] N. El Karoui, I. Karatzas, A New Approach to the Skorohod Problem and its Applications, Stochastics and Stochastics Reports 34 (1991), pp. 57-82.

[26] G. Ferrari, On an Integral Equation for the Free Boundary of Stochastic, Irreversible Investment Problems, Center for Mathematical Economics Working paper no. 471 (2012), arXiv:1211.0412v1. Submitted.

[27] A. Friedman, Partial Differential Equations of Parabolic Type, Dover Publications Inc. 1964.

[28] A. Friedman, Stochastic Games and Variational Inequalities, Archive for Rational Mechanics and Analysis 51 (1973), pp. 321-346.

[29] A. Friedman, Stochastic Differential Equations and Applications, Volume 1, Academic Press 1976.

[30] A. Friedman, Stochastic Differential Equations and Applications, Volume 2, Academic Press 1976.

[31] X. Guo, H. Pham, Optimal Partially Reversible Investment with Entry Decision and General Production Function, Stochastic Processes and their Applications 115 (2005), pp. 705-736.

[32] X. Guo, P. Tomecek, Connections between Singular Control and Optimal Switching, SIAM Journal on Control and Optimization 47(1) (2008), pp. 421-443.

[33] S. Hamadène, J.P. Lepeltier, Zero-sum Stochastic Differential Games and Backward Equations, Systems and Control Letters 24(4) (1995), pp. 259-263.

[34] S. Hamadène, J.P. Lepeltier, Reflected BSDEs and Mixed Game Problem, Stochastic Processes and their Applications 24 (2000), pp. 259-263.

[35] S. Hamadène, M. Jeanblanc, On the Starting and Stopping Problem: Application in Reversible Investments, Mathematics of Operations Research 32(1) (2007), pp. 182-192.

[36] J.M. Harrison, M.I. Taksar, Instantaneous Control of Brownian Motion, Mathematics of Operations Research 8(3) (1983), pp. 439-453.

[37] S. Jacka, Optimal Stopping and the American Put, Mathematical Finance 1 (1991), pp. 1-14.

[38] J. Jacod, Calcul Stochastique et Problèmes de Martingales, no. 714 in Lecture Notes in Mathematics, Springer 1979.

[39] Y. Kabanov, Hedging and Liquidation under Transaction Costs in Currency Markets, Finance and Stochastics 3 (1999), pp. 237-248.

[40] I. Karatzas, A Class of Singular Stochastic Control Problems, Advances in Applied Probability 15 (1983), pp. 225-254. 
[41] I. Karatzas, S.E. Shreve, Connections between Optimal Stopping and Singular Stochastic Control I. Monotone Follower Problems, SIAM Journal on Control and Optimization 22 (1984), pp. 856-877.

[42] I. Karatzas, S.E. Shreve, Brownian Motion and Stochastic Calculus, Springer-Verlag, New York 1988.

[43] I. Karatzas, H. Wang, Connections between Bounded-Variation Control and Dynkin Games in 'Optimal Control and Partial Differential Equations'; Volume in Honor of Professor Alain Bensoussan's 60th Birthday (J.L. Menaldi, A. Sulem and E. Rofman, eds.), pp. 353-362. IOS Press, Amsterdam 2005.

[44] T.Ø. Kobila, A Class of Solvable Stochastic Investment Problems Involving Singular Controls, Stochatics and Stochastic Reports 43 (1993), pp. 29-63.

[45] P. Matomaki, On Solvability of a Two-Sided Singular Control Problem, Mathematical Methods of Operations Research 76(3) (2012), pp. 239-271.

[46] J.L. Menaldi, On the Optimal Stopping Problem for Degenerate Diffusions, SIAM Journal on Control and Optimization 18(6) (1980), pp. 697-721.

[47] A. Merhi, M. Zervos, A Model for Reversible Investment Capacity Expansion, SIAM Journal on Control and Optimization 46(3) (2007), pp. 839-876.

[48] H. Morimoto, Dynkin Games and Martingale Methods, Stochastics 13 (1984), pp. 213-228.

[49] A. Øksendal, Irreversible Investment Problems, Finance and Stochastics 4 (2000), pp. 223250.

[50] G. Peskir, On the American Option problem, Mathematical Finance 15(1) (2005), pp. 169181.

[51] G. Peskir, The Russian Option: Finite Horizon, Finance and Stochastics 9(2) (2005), pp. 251-267.

[52] G. Peskir, A Change-of-Variable Formula with Local Time on Curves, Journal of Theoretical Probability 18(3) (2005), pp. 499-535.

[53] G. Peskir, A. Shyriaev, Optimal Stopping and Free-Boundary Problems, Lectures in Mathematics ETH, Birkhauser 2006.

[54] G. Peskir, Principle of Smooth Fit and Diffusions with Angles, Stochastics 79(3-4) (2007), pp. 293-302.

[55] G. Peskir, Optimal Stopping Games and Nash Equilibrium, SIAM Theory of Probability and its Applications 53(3) (2009), pp. 558-571.

[56] H. Pham, Explicit Solution to an Irreversible Investment Model with a Stochastic Production Capacity, in 'From Stochastic Analysis to Mathematical Finance, Festschrift for Albert Shiryaev' (Y. Kabanov and R. Liptser eds.), Springer 2006.

[57] P. Protter, Stochastic Integration and Differential Equations, Springer, 2004.

[58] D. Revuz, M. Yor, Continuous Martingales and Brownian Motion, Springer-Verlag, Berlin 1999. 
[59] F. Riedel, X. Su, On Irreversible Investment, Finance and Stochastics 15(4) (2011), pp. 607633.

[60] L. Stettner, Zero-sum Markov Games with Stopping and Impulsive Strategies, Applied Mathematics and Optimization 9 (1982), pp. 1-24.

[61] L. Stettner, Penalty Method for Finite Horizon Stopping Problems, SIAM Journal on Control and Optimization 49(3) (2011), pp. 1078-1099.

[62] D.W. Stroock, S.R.S. Varadhan, On Degenerate Elliptic-Parabolic Operators of Second Order and their Associated Diffusions, Communications on Pure and Applied Mathematics 25 (1972), pp. 651-713. 